\title{
EULER CHARACTERISTIC ON NONCOMMUTATIVE POLYBALLS
}

\author{
GELU POPESCU
}

\begin{abstract}
In this paper we introduce and study the Euler characteristic (denoted by $\chi$ ) associated with algebraic modules generated by arbitrary elements of certain noncommutative polyballs. We provide several asymptotic formulas for $\chi$ and prove some of its basic properties. We show that the Euler characteristic is a complete unitary invariant for the finite rank Beurling type invariant subspaces of the tensor product of full Fock spaces $F^{2}\left(H_{n_{1}}\right) \otimes \cdots \otimes F^{2}\left(H_{n_{k}}\right)$, and prove that its range coincides with the interval $[0, \infty)$. We obtain an analogue of Arveson's version of the Gauss-Bonnet-Chern theorem from Riemannian geometry, which connects the curvature to the Euler characteristic. In particular, we prove that if $\mathcal{M}$ is an invariant subspace of $F^{2}\left(H_{n_{1}}\right) \otimes \cdots \otimes F^{2}\left(H_{n_{k}}\right), n_{i} \geq 2$, which is graded (generated by multi-homogeneous polynomials), then the curvature and the Euler characteristic of the orthocomplement of $\mathcal{M}$ coincide.
\end{abstract}

\section{INTRODUCTION}

Arveson ([1, [2], 3]) introduced and studied the curvature invariant and the Euler characteristic for commuting $n$-tuples of bounded linear operators $T=\left(T_{1}, \ldots, T_{n}\right)$ acting on a Hilbert space $\mathcal{H}$ such that $T_{1} T_{1}^{*}+\cdots+T_{n} T_{n}^{*} \leq I$ and the defect operator $\Delta_{T}:=\left(I-T_{1} T_{1}^{*}-\cdots-T_{n} T_{n}^{*}\right)^{1 / 2}$ has finite rank. Based on certain asymptotic formulas for both the curvature and Euler characteristic, he obtained, as the main result of the paper, an operator-theoretic version of the Gauss-Bonnet-Chern formula of Riemannian geometry. More precisely, he proved that for any graded (generated by homogeneous polynomials) invariant subspace $\mathcal{M}$ of a finite direct sum of copies of the symmetric Fock space $F_{s}^{2}\left(H_{n}\right)$ with $n$ generators, the curvature and the Euler characteristic of the othocomplement of $\mathcal{M}$ coincide. This result was generalized by Fang ([6]) to invariant subspaces generated by arbitrary polynomials. Fang also obtained a version of the Gauss-Bonnet-Chern formula for the Hardy space $H^{2}\left(\mathbb{D}^{n}\right)$ over the polydisc $(\underline{8})$ and for the Dirichlet space over the unit disc ([7, 25]). The theory of Arveson's curvature on the symmetric Fock space was significantly expanded due to the work by Greene, Richter, and Sundberg 9], Fang [6], and Gleason, Richter, and Sundberg [10].

In the noncommutative setting, the author (18, 19]) and, independently, Kribs 12 defined and studied the curvature and Euler characteristic for arbitrary elements $T$ with $\operatorname{rank} \Delta_{T}<\infty$ in the unit ball

$$
\left[B(\mathcal{H})^{n}\right]_{1}^{-}:=\left\{\left(X_{1}, \ldots, X_{n}\right) \in B(\mathcal{H})^{n}: X_{1} X_{1}^{*}+\cdots+X_{n} X_{n}^{*} \leq I\right\}
$$

and, in particular, for the invariant (resp. coinvariant) subspaces of the full Fock space $F^{2}\left(H_{n}\right)$ with $n$ generators. Some of these results were extended by Muhly and Solel 13 to a class of completely positive maps on semifinite factors. A noncommutative analogue (for the full Fock space) of the Arveson's version of the Gauss-Bonnet-Chern theorem was obtained in [18.

In 24, we developed a theory of curvature invariant and multiplicity invariant for tensor products of full Fock spaces $F^{2}\left(H_{n_{1}}\right) \otimes \cdots \otimes F^{2}\left(H_{n_{k}}\right)$ and also for tensor products of symmetric Fock spaces $F_{s}^{2}\left(H_{n_{1}}\right) \otimes \cdots \otimes F_{s}^{2}\left(H_{n_{k}}\right)$. To prove the existence of the curvature and its basic properties in these settings required a new approach based on noncommutative Berezin transforms and multivariable operator theory on polyballs and varieties (see [17], 21], 22], and [23]), and also certain summability results for

Date: July 9, 2014 (revised version).

2000 Mathematics Subject Classification. Primary: 46L52; 32A70; Secondary: 47A13; 47A15.

Key words and phrases. Noncommutative polyball; Euler characteristic; Curvature invariant; Berezin transform; Fock space; Creation operators; Invariant subspaces.

Research supported in part by an NSF grant. 
completely positive maps which are trace contractive. In particular, we obtained new proofs for the existence of the curvature on the full Fock space $F^{2}\left(H_{n}\right)$, the Hardy space $H^{2}\left(\mathbb{D}^{k}\right)$ (which corresponds to $\left.n_{1}=\cdots=n_{k}=1\right)$, and the symmetric Fock space $F_{s}^{2}\left(H_{n}\right)$.

In the present paper, which is a continuation of [24, we introduce and study the Euler characteristic associated with the elements of a class of noncommutative polyballs, and obtain an analogue of Arveson's version of the Gauss-Bonnet-Chern theorem from Riemannian geometry, which connects the curvature to the Euler characteristic of some associated algebraic modules.

To present our results we need a few preliminaries. Throughout this paper, we denote by $B(\mathcal{H})$ the algebra of bounded linear operators on a Hilbert space $\mathcal{H}$. Let $B(\mathcal{H})^{n_{1}} \times_{c} \cdots \times_{c} B(\mathcal{H})^{n_{k}}$ be the set of all tuples $\mathbf{X}:=\left(X_{1}, \ldots, X_{k}\right)$ in $B(\mathcal{H})^{n_{1}} \times \cdots \times B(\mathcal{H})^{n_{k}}$ with the property that the entries of $X_{s}:=\left(X_{s, 1}, \ldots, X_{s, n_{s}}\right)$ are commuting with the entries of $X_{t}:=\left(X_{t, 1}, \ldots, X_{t, n_{t}}\right)$ for any $s, t \in\{1, \ldots, k\}$, $s \neq t$. Note that, for each $i \in\{1, \ldots, k\}$, the operators $X_{i, 1}, \ldots, X_{i, n_{i}}$ are not necessarily commuting. Let $\mathbf{n}:=\left(n_{1}, \ldots, n_{k}\right) \in \mathbb{N}^{k}$, where $n_{i} \in \mathbb{N}:=\{1,2, \ldots\}$ and $i \in\{1, \ldots, k\}$, and define the regular polyball

$$
\mathbf{B}_{\mathbf{n}}(\mathcal{H}):=\left\{\mathbf{X}=\left(X_{1}, \ldots, X_{k}\right) \in B(\mathcal{H})^{n_{1}} \times_{c} \cdots \times_{c} B(\mathcal{H})^{n_{k}}: \boldsymbol{\Delta}_{\mathbf{X}}^{\mathbf{p}}(I) \geq 0 \text { for } \mathbf{0} \leq \mathbf{p} \leq(1, \ldots, 1)\right\},
$$

where the defect mapping $\boldsymbol{\Delta}_{\mathbf{X}}^{\mathbf{p}}: B(\mathcal{H}) \rightarrow B(\mathcal{H})$ is defined by

$$
\boldsymbol{\Delta}_{\mathbf{X}}^{\mathbf{p}}:=\left(i d-\Phi_{X_{1}}\right)^{p_{1}} \circ \cdots \circ\left(i d-\Phi_{X_{k}}\right)^{p_{k}}, \quad p_{i} \in\{0,1\},
$$

and $\Phi_{X_{i}}: B(\mathcal{H}) \rightarrow B(\mathcal{H})$ is the completely positive linear map defined by $\Phi_{X_{i}}(Y):=\sum_{j=1}^{n_{i}} X_{i, j} Y X_{i, j}^{*}$ for $Y \in B(\mathcal{H})$. We use the convention that $\left(i d-\Phi_{f_{i}, X_{i}}\right)^{0}=i d$. For information on completely bounded (resp. positive) maps we refer to [14. by

Let $H_{n_{i}}$ be an $n_{i}$-dimensional complex Hilbert space. We consider the full Fock space of $H_{n_{i}}$ defined

$$
F^{2}\left(H_{n_{i}}\right):=\mathbb{C} 1 \oplus \bigoplus_{p \geq 1} H_{n_{i}}^{\otimes p},
$$

where $H_{n_{i}}^{\otimes p}$ is the (Hilbert) tensor product of $p$ copies of $H_{n_{i}}$. Let $\mathbb{F}_{n_{i}}^{+}$be the unital free semigroup on $n_{i}$ generators $g_{1}^{i}, \ldots, g_{n_{i}}^{i}$ and the identity $g_{0}^{i}$. Set $e_{\alpha}^{i}:=e_{j_{1}}^{i} \otimes \cdots \otimes e_{j_{p}}^{i}$ if $\alpha=g_{j_{1}}^{i} \cdots g_{j_{p}}^{i} \in \mathbb{F}_{n_{i}}^{+}$and $e_{g_{0}^{i}}^{i}:=1 \in \mathbb{C}$. Note that $\left\{e_{\alpha}^{i}: \alpha \in \mathbb{F}_{n_{i}}^{+}\right\}$is an orthonormal basis of $F^{2}\left(H_{n_{i}}\right)$. The length of $\alpha \in \mathbb{F}_{n_{i}}^{+}$is defined by $|\alpha|:=0$ if $\alpha=g_{0}^{i}$ and $|\alpha|:=p$ if $\alpha=g_{j_{1}}^{i} \cdots g_{j_{p}}^{i}$, where $j_{1}, \ldots, j_{p} \in\left\{1, \ldots, n_{i}\right\}$.

For each $n_{i} \in \mathbb{N}, i \in\{1, \ldots, k\}$, and $j \in\left\{1, \ldots, n_{i}\right\}$, let $S_{i, j}: F^{2}\left(H_{n_{i}}\right) \rightarrow F^{2}\left(H_{n_{i}}\right)$ be the left creation operator defined by $S_{i, j} e_{\alpha}^{i}:=e_{g_{j} \alpha}^{i}, \alpha \in \mathbb{F}_{n_{i}}^{+}$, and define the operator $\mathbf{S}_{i, j}$ acting on the tensor Hilbert space $F^{2}\left(H_{n_{1}}\right) \otimes \cdots \otimes F^{2}\left(H_{n_{k}}\right)$ by setting

$$
\mathbf{S}_{i, j}:=\underbrace{I \otimes \cdots \otimes I}_{i-1 \text { times }} \otimes S_{i, j} \otimes \underbrace{I \otimes \cdots \otimes I}_{k-i \text { times }} .
$$

The $k$-tuple $\mathbf{S}:=\left(\mathbf{S}_{1}, \ldots, \mathbf{S}_{k}\right)$, where $\mathbf{S}_{i}:=\left(\mathbf{S}_{i, 1}, \ldots, \mathbf{S}_{i, n_{i}}\right)$, is an element in the regular polyball $\mathbf{B}_{\mathbf{n}}\left(\otimes_{i=1}^{k} F^{2}\left(H_{n_{i}}\right)\right)$ and it is called universal model associated with the abstract polyball $\mathbf{B}_{\mathbf{n}}:=\left\{\mathbf{B}_{\mathbf{n}}(\mathcal{H})\right.$ : $\mathcal{H}$ is a Hilbert space $\}$. The noncommutative Hardy algebra $F^{\infty}\left(\mathbf{B}_{\mathbf{n}}\right)$ is the sequential SOT-(resp. WOT-, $w^{*}$-) closure of all polynomials in $\mathbf{S}_{i, j}$ and the identity.

Let $\mathbf{T}=\left(T_{1}, \ldots, T_{k}\right) \in \mathbf{B}_{\mathbf{n}}(\mathcal{H})$ with $T_{i}:=\left(T_{i, 1}, \ldots, T_{i, n_{i}}\right)$. We use the notation $T_{i, \alpha_{i}}:=T_{i, j_{1}} \cdots T_{i, j_{p}}$ if $\alpha_{i} \in \mathbb{F}_{n_{i}}^{+}$and $\alpha_{i}=g_{j_{1}}^{i} \cdots g_{j_{p}}^{i}$, and $T_{i, g_{0}^{i}}:=I$. The noncommutative Berezin kernel associated with any element $\mathbf{T}$ in the noncommutative polyball $\mathbf{B}_{\mathbf{n}}(\mathcal{H})$ is the operator

$$
\mathbf{K}_{\mathbf{T}}: \mathcal{H} \rightarrow F^{2}\left(H_{n_{1}}\right) \otimes \cdots \otimes F^{2}\left(H_{n_{k}}\right) \otimes \overline{\mathbf{\Delta}_{\mathbf{T}}(I)(\mathcal{H})}
$$

defined by

$$
\mathbf{K}_{\mathbf{T}} h:=\sum_{\beta_{i} \in \mathbb{F}_{n_{i}}^{+}, i=1, \ldots, k} e_{\beta_{1}}^{1} \otimes \cdots \otimes e_{\beta_{k}}^{k} \otimes \boldsymbol{\Delta}_{\mathbf{T}}(I)^{1 / 2} T_{1, \beta_{1}}^{*} \cdots T_{k, \beta_{k}}^{*} h, \quad h \in \mathcal{H},
$$

where the defect operator is defined by $\boldsymbol{\Delta}_{\mathbf{T}}(I):=\left(i d-\Phi_{T_{1}}\right) \cdots\left(i d-\Phi_{T_{k}}\right)(I)$. The noncommutative Berezin kernel $\mathbf{K}_{\mathbf{T}}$ is a contraction and $\mathbf{K}_{\mathbf{T}} T_{i, j}^{*}=\left(\mathbf{S}_{i, j}^{*} \otimes I\right) \mathbf{K}_{\mathbf{T}}$ for any $i \in\{1, \ldots, k\}$ and $j \in\left\{1, \ldots, n_{i}\right\}$. More about the theory of noncommutative Berezin kernels on polybals and polydomains can be found in [17, 20], 21], 22], and [23]. 
In a recent paper 24, we introduced the curvature of any element $\mathbf{T} \in \mathbf{B}_{\mathbf{n}}(\mathcal{H})$ with trace class defect, i.e. $\operatorname{trace}\left[\boldsymbol{\Delta}_{\mathbf{T}}(I)\right]<\infty$, by setting

$$
\operatorname{curv}(\mathbf{T}):=\lim _{m \rightarrow \infty} \frac{1}{\left(\begin{array}{c}
m+k \\
k
\end{array}\right)} \sum_{\substack{q_{1} \geq 0, \ldots, q_{k} \geq 0 \\
q_{1}+\cdots, q_{k} \leq m}} \frac{\operatorname{trace}\left[\mathbf{K}_{\mathbf{T}}^{*}\left(P_{q_{1}}^{(1)} \otimes \cdots \otimes P_{q_{k}}^{(k)} \otimes I_{\mathcal{H}}\right) \mathbf{K}_{\mathbf{T}}\right]}{\operatorname{trace}\left[P_{q_{1}}^{(1)} \otimes \cdots \otimes P_{q_{k}}^{(k)}\right]},
$$

where $\mathbf{K}_{\mathbf{T}}$ is the Berezin kernel of $\mathbf{T}$ and $P_{q_{i}}^{(i)}$ is the orthogonal projection of the full Fock space $F^{2}\left(H_{n_{i}}\right)$ onto the span of all vectors $e_{\alpha_{i}}^{i}$ with $\alpha \in \mathbb{F}_{n_{i}}^{+}$and $\left|\alpha_{i}\right|=q_{i}$. The curvature is a unitary invariant that measures how far $\mathbf{T}$ is from being "free", i.e. a multiple of the universal model $\mathbf{S}$. We proved the existence of the curvature and established many asymptotic formulas and its basic properties. These results were used to develop a theory of curvature (resp. multiplicity) invariant for tensor products of full Fock spaces $F^{2}\left(H_{n_{1}}\right) \otimes \cdots \otimes F^{2}\left(H_{n_{k}}\right)$ and also for tensor products of symmetric Fock spaces $F_{s}^{2}\left(H_{n_{1}}\right) \otimes \cdots \otimes F_{s}^{2}\left(H_{n_{k}}\right)$.

Throughout the present paper, unless otherwise specified, we assume that $\mathbf{n}:=\left(n_{1}, \ldots, n_{k}\right) \in \mathbb{N}^{k}$ with $n_{i} \geq 2$. Let $\mathbf{T}=\left(T_{1}, \ldots, T_{k}\right) \in B(\mathcal{H})^{n_{1}} \times_{c} \cdots \times_{c} B(\mathcal{H})^{n_{k}}$ be a $k$-tuple such that its defect operator $\boldsymbol{\Delta}_{\mathbf{T}}(I)$ has finite rank. For each $\mathbf{q}=\left(q_{1}, \ldots, q_{k}\right) \in \mathbb{Z}_{+}^{k}$, where $\mathbb{Z}_{+}:=\{0\} \cup \mathbb{N}$, we define the linear manifold

$$
M_{\mathbf{q}}(\mathbf{T}):=\operatorname{span}\left\{T_{1, \alpha_{1}} \cdots T_{k, \alpha_{k}} h: \alpha_{i} \in \mathbb{F}_{n_{i}}^{+},\left|\alpha_{i}\right| \leq q_{i}, h \in \boldsymbol{\Delta}_{\mathbf{T}}(I)(\mathcal{H})\right\} .
$$

In Section 1, we introduce the Euler characteristic of $\mathbf{T}$ by setting

$$
\chi(\mathbf{T}):=\lim _{m \rightarrow \infty} \frac{\operatorname{dim} M_{\mathbf{q}^{(m)}}(\mathbf{T})}{\prod_{i=1}^{k}\left(1+n_{i}+\cdots+n_{i}^{q_{i}^{(m)}}\right)},
$$

where $\mathbf{q}^{(m)}=\left(q_{1}^{(m)}, \ldots, q_{k}^{(m)}\right)$ is a cofinal sequence in $\mathbb{Z}_{+}^{k}$. We show the Euler characteristic exists and provide several asymptotic formulas including the following:

$$
\begin{aligned}
\chi(\mathbf{T}) & =\lim _{\mathbf{q}=\left(q_{1}, \ldots, q_{k}\right) \in \mathbb{Z}_{+}^{k}} \frac{\operatorname{rank}\left[\mathbf{K}_{\mathbf{T}}^{*}\left(P_{\leq\left(q_{1}, \ldots, q_{k}\right)} \otimes I\right) \mathbf{K}_{\mathbf{T}}\right]}{\operatorname{rank}\left[P_{\leq\left(q_{1}, \ldots, q_{k}\right)}\right]} \\
& =\lim _{\mathbf{q}=\left(q_{1}, \ldots, q_{k}\right) \in \mathbb{Z}_{+}^{k}} \frac{\operatorname{rank}\left[\left(i d-\Phi_{T_{1}}^{q_{1}+1}\right) \circ \cdots \circ\left(i d-\Phi_{T_{k}}^{q_{k}+1}\right)(I)\right]}{\prod_{i=1}^{k}\left(1+n_{i}+\cdots+n_{i}^{q_{i}}\right)},
\end{aligned}
$$

where $\mathbf{K}_{\mathbf{T}}$ is the Berezin kernel and $\Phi_{T_{1}}, \ldots, \Phi_{T_{k}}$ are the completely positive maps associated with $\mathbf{T}$. It turns out that

$$
0 \leq \operatorname{curv}(\mathbf{T}) \leq \chi(\mathbf{T}) \leq \operatorname{rank}\left[\boldsymbol{\Delta}_{\mathbf{T}}(I)\right]
$$

We remark that the condition $n_{i} \geq 2$ for all $i \in\{1, \ldots, k\}$ is needed to prove the existence of the Euler characteristic (see the proof of Theorem 1.1). However, there are indications that the result remains true if one ore more of the $n_{i}$ 's assume the value one. For now, this remains an open problem. We should add the fact that the curvature invariant exists if $n_{i} \geq 1$ (see [24]). On the other hand, an important question that remains open is whether one can drop the condition that $T_{i, j}$ commutes with $T_{s, t}$ for $i \neq s$.

We say that $\mathcal{M}$ is an invariant subspace of the tensor product $F^{2}\left(H_{n_{1}}\right) \otimes \cdots \otimes F^{2}\left(H_{n_{k}}\right) \otimes \mathcal{H}$ or that $\mathcal{M}$ is invariant under $\mathbf{S} \otimes I_{\mathcal{H}}$ if it is invariant under each operator $\mathbf{S}_{i, j} \otimes I_{\mathcal{H}}$. When $\left.\left(\mathbf{S} \otimes I_{\mathcal{H}}\right)\right|_{\mathcal{M}}$ has finite rank defect, we say that $\mathcal{M}$ has finite rank. Given two invariant subspaces $\mathcal{M}$ and $\mathcal{N}$ under $\mathbf{S} \otimes I_{\mathcal{H}}$, we say that they are unitarily equivalent if there is a unitary operator $U: \mathcal{M} \rightarrow \mathcal{N}$ such that $\left.U\left(\mathbf{S}_{i, j} \otimes I_{\mathcal{H}}\right)\right|_{\mathcal{M}}=\left.\left(\mathbf{S}_{i, j} \otimes I_{\mathcal{H}}\right)\right|_{\mathcal{N}} U$.

In Section 2, we present a more direct and more transparent proof for the characterization of the invariant subspaces of $\otimes_{i=1}^{k} F^{2}\left(H_{n_{i}}\right)$ with positive defect operator, i.e $\Delta_{\mathcal{M}}:=\boldsymbol{\Delta}_{\mathbf{S} \otimes I_{\mathcal{H}}}\left(P_{\mathcal{M}}\right) \geq 0$. This result complements the corresponding one from [22] and brings new insight concerning the structure of the invariant subspaces of the tensor product $\otimes_{i=1}^{k} F^{2}\left(H_{n_{i}}\right)$. The invariant subspaces with positive defect operators are the so-called Beurling type invariant subspaces.

We show that the Euler characteristic completely classifies the finite rank Beurling type invariant subspaces of $\mathbf{S} \otimes I_{\mathcal{H}}$ which do not contain reducing subspaces. In particular, the Euler characteristic classifies the finite rank Beurling type invariant subspaces of $F^{2}\left(H_{n_{1}}\right) \otimes \cdots \otimes F^{2}\left(H_{n_{k}}\right)$ (see Theorem 2.3). 
Let $\mathcal{M}$ be an invariant subspace of the tensor product $F^{2}\left(H_{n_{1}}\right) \otimes \cdots \otimes F^{2}\left(H_{n_{k}}\right) \otimes \mathcal{E}$, where $\mathcal{E}$ is a finite dimensional Hilbert space. We introduce the Euler characteristic of the orthocomplement $\mathcal{M}^{\perp}$ by setting

$$
\chi\left(\mathcal{M}^{\perp}\right):=\lim _{q_{1} \rightarrow \infty} \cdots \lim _{q_{k} \rightarrow \infty} \frac{\operatorname{rank}\left[P_{\mathcal{M}^{\perp}}\left(P_{\leq\left(q_{1}, \ldots, q_{k}\right)} \otimes I_{\mathcal{E}}\right)\right]}{\operatorname{rank}\left[P_{\leq\left(q_{1}, \ldots, q_{k}\right)}\right]},
$$

where $P_{\leq\left(q_{1}, \ldots, q_{k}\right)}$ is the orthogonal projection of $\otimes_{i=1}^{k} F^{2}\left(H_{n_{i}}\right)$ onto the span of all vectors of the form $e_{\alpha_{1}}^{1} \otimes \cdots \otimes e_{\alpha_{k}}^{k}$, where $\alpha_{i} \in \mathbb{F}_{n_{i}}^{+},\left|\alpha_{i}\right| \leq q_{i}$. In Section 2, we show that the Euler characteristic of $\mathcal{M}^{\perp}$ exists and satisfies the equation

$$
\chi\left(\mathcal{M}^{\perp}\right)=\chi(\mathbf{M})
$$

where $\mathbf{M}$ is the compression of $\mathbf{S} \otimes I_{\mathcal{E}}$ to the orthocomplement of $\mathcal{M}$. This is used to show that for any $t \in[0,1]$ there is an invariant subspace $\mathcal{M}(t)$ of $F^{2}\left(H_{n_{1}}\right) \otimes \cdots \otimes F^{2}\left(H_{n_{k}}\right)$ such that $\chi\left(\mathcal{M}(t)^{\perp}\right)=t$ and, consequently, the range of the Euler characteristic coincides with the interval $[0, \infty)$. If $k=1$, the corresponding result was proved in [18] and [12]. Moreover, if $k \geq 2$, we prove that for each $t \in(0,1)$, there exists an uncountable family $\left\{T^{(\omega)}(t)\right\} \omega \in \Omega$ of non-isomorphic pure elements of rank one defect in the regular polyball such that

$$
\chi\left(T^{(\omega)}(t)\right)=t, \quad \text { for all } \omega \in \Omega .
$$

In Section 3, we provide a characterization of the graded invariant subspaces of $F^{2}\left(H_{n_{1}}\right) \otimes \cdots \otimes F^{2}\left(H_{n_{k}}\right)$ with positive defect operator $\Delta_{\mathcal{M}}$. We also prove that if $\mathcal{M}$ is any graded invariant subspace of the tensor product $F^{2}\left(H_{n_{1}}\right) \otimes \cdots \otimes F^{2}\left(H_{n_{k}}\right)$, then

$$
\lim _{q_{1} \rightarrow \infty} \cdots \lim _{q_{k} \rightarrow \infty} \frac{\operatorname{rank}\left[P_{\mathcal{M}} \perp P_{\leq\left(q_{1}, \ldots, q_{k}\right)}\right]}{\operatorname{rank}\left[P_{\leq\left(q_{1}, \ldots, q_{k}\right)}\right]}=\lim _{q_{1} \rightarrow \infty} \cdots \lim _{q_{k} \rightarrow \infty} \frac{\operatorname{trace}\left[P_{\mathcal{M}} P_{\leq\left(q_{1}, \ldots, q_{k}\right)}\right]}{\operatorname{trace}\left[P_{\leq\left(q_{1}, \ldots, q_{k}\right)}\right]} .
$$

This is equivalent to

$$
\chi\left(\left.P_{\mathcal{M}^{\perp}} \mathbf{S}\right|_{\mathcal{M}^{\perp}}\right)=\operatorname{curv}\left(\left.P_{\mathcal{M} \perp} \mathbf{S}\right|_{\mathcal{M}^{\perp}}\right),
$$

which is an analogue of Arveson's version [2] of the Gauss-Bonnet-Chern theorem from Riemannian geometry in our setting. We recall 22 that any pure element with rank-one defect in the polyball $\mathbf{B}_{\mathbf{n}}(\mathcal{H})$ has the form $\left.P_{\mathcal{M}^{\perp}} \mathbf{S}\right|_{\mathcal{M}^{\perp}}$, where $\mathcal{M}$ is an invariant subspace of the tensor product $F^{2}\left(H_{n_{1}}\right) \otimes \cdots \otimes F^{2}\left(H_{n_{k}}\right)$. It remains an open problem whether, as in the commutative setting ([6], 7]), the result above holds true for any invariant subspace $\mathcal{M}$ generated by arbitrary polynomials. We mention that a version of the Gauss-Bonnet-Chern theorem for graded pure elements of finite rank defect in the noncommutative polyball is also obtained. Finally, we remark that the results of this paper can be re-formulated in terms of Hilbert modules [5] over the complex semigroup algebra $\mathbb{C}\left[\mathbb{F}_{n_{1}}^{+} \times \cdots \times \mathbb{F}_{n_{k}}^{+}\right]$generated by direct product of free semigroups. In this setting, the Hilbert module associated with the universal model of the abstract polyball $\mathbf{B}_{\mathbf{n}}$ plays the role of rank-one free module in the algebraic theory [1].

\section{EULER CHARACTERISTIC ON NONCOMMUTATIVE POLYBALlS}

In this section we prove the existence of the Euler characteristic of any element $\mathbf{T}$ in the noncommutative polyball and provide several asymptotic formulas in terms of the noncommutative Berezin kernel and the completely positive maps associated with $\mathbf{T} \in \mathbf{B}_{\mathbf{n}}(\mathcal{H})$. Basic properties of the Euler characteristic are also proved.

Let $\mathbf{T}=\left(T_{1}, \ldots, T_{k}\right) \in B(\mathcal{H})^{n_{1}} \times_{c} \cdots \times_{c} B(\mathcal{H})^{n_{k}}$ with $T_{i}:=\left(T_{i, 1}, \ldots, T_{i, n_{i}}\right)$, and let $\mathcal{D} \subset \mathcal{H}$ be a finite dimensional subspace. For each $\mathbf{q}=\left(q_{1}, \ldots, q_{k}\right) \in \mathbb{Z}_{+}^{k}$, where $\mathbb{Z}_{+}=\{0,1, \ldots\}$, we define

$$
M_{\mathbf{q}}(\mathbf{T}, \mathcal{D}):=\operatorname{span}\left\{T_{1, \alpha_{1}} \cdots T_{k, \alpha_{k}} h: \alpha_{i} \in \mathbb{F}_{n_{i}}^{+},\left|\alpha_{i}\right| \leq q_{i}, h \in \mathcal{D}\right\} .
$$

We also use the notation $M_{\left(q_{1}, \ldots, q_{k}\right)}$ when $\mathbf{T}$ and $\mathcal{D}$ are understood and we want to emphasis the coordinates $q_{1}, \ldots q_{k}$. Given two $k$-tuples $\mathbf{q}=\left(q_{1}, \ldots, q_{k}\right)$ and $\mathbf{p}=\left(p_{1}, \ldots, p_{k}\right)$ in $\mathbb{Z}_{+}^{k}$, we consider the partial order $\mathbf{q} \leq \mathbf{p}$ defined by $q_{i} \leq p_{i}$ for any $i \in\{1, \ldots, k\}$. We consider $\mathbb{Z}_{+}^{k}$ as a directed set with respect to this partial order. 
Theorem 1.1. Let $\mathcal{D} \subset \mathcal{H}$ be a finite dimensional subspace and let $\mathbf{T} \in B(\mathcal{H})^{n_{1}} \times_{c} \cdots \times_{c} B(\mathcal{H})^{n_{k}}$, where $n_{i} \geq 2$ for any $i \in\{1, \ldots, k\}$. Then the following iterated limits exist and are equal

$$
\lim _{q_{\sigma(1)} \rightarrow \infty} \cdots \lim _{q_{\sigma(k)} \rightarrow \infty} \frac{\operatorname{dim} M_{\mathbf{q}}(\mathbf{T}, \mathcal{D})}{n_{1}^{q_{1}} \cdots n_{k}^{q_{k}}},
$$

where $\sigma$ is any permutation of $\{1, \ldots, k\}$ and $\mathbf{q}=\left(q_{1}, \ldots, q_{k}\right) \in \mathbb{Z}_{+}^{k}$. Moreover, these limits coincide with

$$
\lim _{\mathbf{q}=\left(q_{1}, \ldots, q_{k}\right) \in \mathbb{Z}_{+}^{k}} \frac{\operatorname{dim} M_{\mathbf{q}}(\mathbf{T}, \mathcal{D})}{n_{1}^{q_{1}} \cdots n_{k}^{q_{k}}} .
$$

Proof. If $\mathbf{q}=\left(q_{1}, \ldots, q_{k}\right) \in \mathbb{Z}_{+}^{k}$, we use the notation $M_{\left(q_{1}, \ldots, q_{k}\right)}:=M_{\mathbf{q}}(\mathbf{T}, \mathcal{D})$. For $i \in\{1, \ldots, k\}$, note that

$$
M_{\left(q_{1}, \ldots, q_{k}\right)}=M_{\left(q_{1}, \ldots q_{i-1}, 0, q_{i+1} \ldots, q_{k}\right)}+T_{i, 1} M_{\left(q_{1}, \ldots q_{i-1}, q_{i}-1, q_{i+1} \ldots, q_{k}\right)}+\cdots+T_{i, n_{i}} M_{\left(q_{1}, \ldots q_{i-1}, q_{i}-1, q_{i+1} \ldots, q_{k}\right)} .
$$

Consequently, we deduce that

$$
\operatorname{dim} M_{\left(q_{1}, \ldots, q_{k}\right)} \leq n_{i} \operatorname{dim} M_{\left(q_{1}, \ldots q_{i-1}, q_{i}-1, q_{i+1} \ldots, q_{k}\right)}+\operatorname{dim} M_{\left(q_{1}, \ldots q_{i-1}, 0, q_{i+1} \ldots, q_{k}\right)}
$$

for any $\left(q_{1}, \ldots, q_{k}\right) \in \mathbb{Z}_{+}^{k}$. Iterating this inequality, we obtain

$$
\operatorname{dim} M_{\left(q_{1}, \ldots, q_{k}\right)} \leq\left(1+n_{i}+\cdots+n_{i}^{q_{i}}\right) \operatorname{dim} M_{\left(q_{1}, \ldots q_{i-1}, 0, q_{i+1} \ldots, q_{k}\right)},
$$

which implies

$$
\frac{\operatorname{dim} M_{\left(q_{1}, \ldots, q_{k}\right)}}{\prod_{i=1}^{k}\left(1+n_{i}+\cdots+n_{i}^{q_{i}}\right)} \leq \operatorname{dim} M_{(0, \ldots, 0)}=\operatorname{dim} \mathcal{D}
$$

for any $\left(q_{1}, \ldots, q_{k}\right) \in \mathbb{Z}_{+}^{k}$. On the other hand, relations (1.1), (1.2), and (1.3) imply

$$
0 \leq \frac{\operatorname{dim} M_{\left(q_{1}, \ldots, q_{k}\right)}}{n_{i}^{q_{i}}} \leq \frac{\operatorname{dim} M_{\left(q_{1}, \ldots q_{i-1}, q_{i}-1, q_{i+1} \ldots, q_{k}\right)}}{n_{i}^{q_{i}-1}}+\frac{1}{n_{i}^{q_{i}}} \prod_{\substack{s=1 \\ s \neq i}}^{k}\left(1+n_{s}+\cdots+n_{s}^{q_{s}}\right) \operatorname{dim} \mathcal{D} .
$$

For $x \in \mathbb{R}$ we set $x^{+}:=\max \{x, 0\}$ and $x^{-}:=\max \{-x, 0\}$. Denote $x_{q_{i}}:=\frac{\operatorname{dim} M_{\left(q_{1}, \ldots, q_{k}\right)}}{n_{i}^{q_{i}}}$ and note that

$$
0 \leq x_{N}=x_{0}+\sum_{p=1}^{N}\left(x_{p}-x_{p-1}\right)^{+}-\sum_{p=1}^{N}\left(x_{p}-x_{p-1}\right)^{-} .
$$

Hence, using relation (1.4), we deduce that

$$
\sum_{p=1}^{N}\left(x_{p}-x_{p-1}\right)^{-} \leq x_{0}+\sum_{p=1}^{N}\left(x_{p}-x_{p-1}\right)^{+} \leq x_{0}+\left(\sum_{p=1}^{N} \frac{1}{n_{i}^{p}}\right) \prod_{\substack{s=1 \\ s \neq i}}^{k}\left(1+n_{s}+\cdots+n_{s}^{q_{s}}\right) \operatorname{dim} \mathcal{D} .
$$

Consequently, we obtain

$$
x_{0}+\sum_{p=1}^{N}\left|x_{p}-x_{p-1}\right| \leq 2 x_{0}+2 \sum_{p=1}^{N}\left(x_{p}-x_{p-1}\right)^{+}
$$

for any $N \in \mathbb{N}$. Since $n_{i} \geq 2$, the inequalities above show that the sequence $x_{N}=x_{0}+\sum_{p=1}^{N}\left(x_{p}-x_{p-1}\right)$ is convergent as $N \rightarrow \infty$. Therefore,

$$
\lim _{q_{i} \rightarrow \infty} \frac{\operatorname{dim} M_{\left(q_{1}, \ldots, q_{k}\right)}}{n_{i}^{q_{i}}} \quad \text { exists for any } \quad q_{1}, \ldots q_{i-1}, q_{i+1} \ldots, q_{k} \in \mathbb{Z}_{+} .
$$

The next step in our proof is to show that the iterated limit

$$
\lim _{q_{k} \rightarrow \infty} \cdots \lim _{q_{1} \rightarrow \infty} \frac{\operatorname{dim} M_{\left(q_{1}, \ldots, q_{k}\right)}}{n_{1}^{q_{1}} \cdots n_{k}^{q_{k}}} \quad \text { exists. }
$$


We use an inductive argument. Due to relation (1.5), the $\operatorname{limit} \lim _{q_{1} \rightarrow \infty} \frac{\operatorname{dim} M_{\left(q_{1}, \ldots, q_{k}\right)}}{n_{1}^{q_{1}}}$ exists for any $q_{2}, \ldots, q_{k}$ in $\mathbb{Z}_{+}$. Assume that $1 \leq p \leq k-1$ and that the iterated limit

$$
y\left(q_{p+1}, \ldots, q_{k}\right):=\lim _{q_{p} \rightarrow \infty} \cdots \lim _{q_{1} \rightarrow \infty} \frac{\operatorname{dim} M_{\left(q_{1}, \ldots, q_{k}\right)}}{n_{1}^{q_{1}} \cdots n_{p}^{q_{p}}}
$$

exists for any $q_{p+1}, \ldots, q_{k}$ in $\mathbb{Z}_{+}$. Due to relation (1.4), we have

$$
0 \leq \frac{\operatorname{dim} M_{\left(q_{1}, \ldots, q_{k}\right)}}{n_{1}^{q_{1}} \cdots n_{p}^{q_{p}}} \leq n_{p+1} \frac{\operatorname{dim} M_{\left(q_{1}, \ldots q_{p}, q_{p+1}-1, q_{p+2} \ldots, q_{k}\right)}}{n_{1}^{q_{1}} \cdots n_{p}^{q_{p}}}+\frac{1}{n_{1}^{q_{1}} \cdots n_{p}^{q_{p}}} \prod_{\substack{s=1 \\ s \neq p+1}}^{k}\left(1+n_{s}+\cdots+n_{s}^{q_{s}}\right) \operatorname{dim} \mathcal{D} .
$$

Consequently, taking the limits as $q_{1} \rightarrow \infty, \ldots, q_{p} \rightarrow \infty$, we deduce that

$$
\frac{y\left(q_{p+1}, \ldots, q_{k}\right)}{n_{p+1}^{q_{p+1}}} \leq \frac{y\left(q_{p+1}-1, q_{p+2}, \ldots, q_{k}\right)}{n_{p+1}^{q_{p+1}-1}}+\frac{1}{n_{p+1}^{q_{p+1}}} \prod_{s=1}^{p} \frac{n_{s}}{n_{s}-1} \prod_{s=p+2}^{k}\left(1+n_{s}+\cdots+n_{s}^{q_{s}}\right) \operatorname{dim} \mathcal{D} .
$$

Setting $z_{q_{p+1}}:=\frac{y\left(q_{p+1}, \ldots, q_{k}\right)}{n_{p+1}^{q_{p+1}}}$, we have

$$
0 \leq z_{q_{p+1}} \leq z_{q_{p+1}-1}+\frac{1}{n_{p+1}^{q_{p+1}}} \prod_{s=1}^{p} \frac{n_{s}}{n_{s}-1} \prod_{s=p+2}^{k}\left(1+n_{s}+\cdots+n_{s}^{q_{s}}\right) \operatorname{dim} \mathcal{D}, \quad q_{p+1} \in \mathbb{N}
$$

Similarly to the proof that $x_{q_{i}}$ is convergent (see relation (1.5)), one can show that the sequence $\left\{z_{q_{p+1}}\right\}$ is convergent as $q_{p+1} \rightarrow \infty$ for any $q_{p+2}, \ldots, q_{k}$ in $\mathbb{Z}_{+}$. Therefore, the iterated limit

$$
y\left(q_{p+2}, \ldots, q_{k}\right):=\lim _{q_{p+1} \rightarrow \infty} \cdots \lim _{q_{1} \rightarrow \infty} \frac{\operatorname{dim} M_{\left(q_{1}, \ldots, q_{k}\right)}}{n_{1}^{q_{1}} \cdots n_{p+1}^{q_{p+1}}} \quad \text { exists, }
$$

which proves our assertion. Since the entries of $X_{s}:=\left(X_{s, 1}, \ldots, X_{s, n_{s}}\right)$ are commuting with the entries of $X_{t}:=\left(X_{t, 1}, \ldots, X_{t, n_{t}}\right)$ for any $s, t \in\{1, \ldots, k\}, s \neq t$, similar arguments as above show that, for any permutation $\sigma$ of $\{1, \ldots, k\}$, the iterated limit $L_{\sigma}:=\lim _{q_{\sigma(1)} \rightarrow \infty} \cdots \lim _{q_{\sigma(k)} \rightarrow \infty} a_{\left(q_{1}, \ldots, q_{k}\right)}$ exists, where $a_{\left(q_{1}, \ldots, q_{k}\right)}:=\frac{\operatorname{dim} M_{\left(q_{1}, \ldots, q_{k}\right)}}{n_{1}^{q_{1} \ldots n_{k}^{q_{k}}}}$.

The next step is to show that all these iterated limits are equal. We proceed by contradiction. Without loss of generality we can assume that $L_{i d}<L_{\sigma}$ for some permutation $\sigma$. Let $\epsilon>0$ be such that $\epsilon<\frac{L_{\sigma}-L_{i d}}{4}$. Since $n_{i} \geq 2$ and $L_{i d}=\lim _{q_{1} \rightarrow \infty} \cdots \lim _{q_{k} \rightarrow \infty} a_{\left(q_{1}, \ldots, q_{k}\right)}$, we can choose natural numbers $N_{1} \leq N_{2} \leq \ldots \leq N_{k}$ such that $\sum_{j=N_{i}+1}^{\infty} \frac{1}{n_{i}^{j}}<\frac{\epsilon}{k M}$ for any $i \in\{1, \ldots, k\}$, where $M:=(\operatorname{dim} \mathcal{D}) \prod_{i=1}^{k} \frac{n_{i}}{n_{i}-1}$, and such that

$$
\left|a_{\left(N_{1}, \ldots, N_{k}\right)}-L_{i d}\right|<\epsilon .
$$

Taking into account that $L_{\sigma}=\lim _{q_{\sigma(1)} \rightarrow \infty} \cdots \lim _{q_{\sigma(k)} \rightarrow \infty} a_{\left(q_{1}, \ldots, q_{k}\right)}$, we can choose natural numbers $C_{1}, \ldots, C_{k}$ such that $C_{i}>\max \left\{N_{1}, \ldots, N_{k}\right\}$ and

$$
\left|a_{\left(C_{1}, \ldots, C_{k}\right)}-L_{\sigma}\right|<\epsilon .
$$

Consequently, we obtain

$$
a_{\left(C_{1}, \ldots, C_{k}\right)}-a_{\left(N_{1}, \ldots, N_{k}\right)}>2 \epsilon .
$$

For each $\left(q_{1}, \ldots, q_{k}\right) \in \mathbb{Z}_{+}^{k}$, set

$$
d_{\left(q_{1}, \ldots, q_{k}\right)}:=\frac{1}{n_{1}^{q_{1}} \cdots n_{k}^{q_{k}}} \prod_{i=1}^{k}\left(1+n_{i}+\cdots n_{i}^{q_{i}}\right) \operatorname{dim} \mathcal{D}
$$


and note that $d_{\left(q_{1}, \ldots, q_{k}\right)} \leq(\operatorname{dim} \mathcal{D}) \prod_{i=1}^{k} \frac{n_{i}}{n_{i}-1}$. Using relation (1.1), we deduce that

$$
\begin{aligned}
& a_{\left(C_{1}, C_{2}, \ldots, C_{k}\right)} \\
& =a_{\left(N_{1}, N_{2}, \ldots, N_{k}\right)}+\left[\left(a_{\left(N_{1}+1, N_{2}, \ldots, N_{k}\right)}-a_{\left(N_{1}, N_{2}, \ldots, N_{k}\right)}\right)+\cdots+\left(a_{\left(C_{1}, N_{2}, \ldots, N_{k}\right)}-a_{\left(C_{1}-1, N_{2}, \ldots, N_{k}\right)}\right)\right] \\
& +\left[\left(a_{\left(C_{1}, N_{2}+1, N_{3}, \ldots, N_{k}\right)}-a_{\left(C_{1}, N_{2}, N_{3}, \ldots, N_{k}\right)}\right)+\cdots+\left(a_{\left(C_{1}, C_{2}, N_{3}, \ldots, N_{k}\right)}-a_{\left(C_{1}, C_{2}-1, N_{3} \ldots, N_{k}\right)}\right)\right] \\
& \vdots \\
& +\left[\left(a_{\left(C_{1}, C_{2}, \ldots, C_{k-1}, N_{k}+1\right)}-a_{\left(C_{1}, C_{2}, \ldots, C_{k-1}, N_{k}\right)}\right)+\cdots+\left(a_{\left(C_{1}, C_{2}, \ldots, C_{k}\right)}-a_{\left(C_{1}, C_{2}, \ldots, C_{k-1}, C_{k}-1\right)}\right)\right] \\
& \leq a_{\left(N_{1}, N_{2}, \ldots, N_{k}\right)}+\sum_{j=N_{1}+1}^{C_{1}} \frac{1}{n_{1}^{j}} \frac{\operatorname{dim} M_{\left(0, N_{2}, \ldots, N_{k}\right)}}{n_{2}^{N_{2}} \cdots n_{k}^{N_{k}}} \\
& +\sum_{j=N_{2}+1}^{C_{2}} \frac{1}{n_{2}^{j}} \frac{\operatorname{dim} M_{\left(C_{1}, 0, N_{3}, \ldots, N_{k}\right)}}{n_{1}^{C_{1}} n_{3}^{N_{3}} \cdots n_{k}^{N_{k}}}+\cdots+\sum_{j=N_{k}+1}^{C_{k}} \frac{1}{n_{k}^{j}} \frac{\operatorname{dim} M_{\left(C_{1}, 0, N_{3}, \ldots, N_{k}\right)}}{n_{1}^{C_{1}} n_{2}^{N_{2}} \cdots n_{k-1}^{N_{k}-1}} \\
& \leq a_{\left(N_{1}, N_{2}, \ldots, N_{k}\right)}+d_{\left(0, N_{2}, \ldots, N_{k}\right)}\left(\sum_{j=N_{1}+1}^{C_{1}} \frac{1}{n_{1}^{j}}\right) \\
& +d_{\left(C_{1}, 0, N_{3}, \ldots, N_{k}\right)}\left(\sum_{j=N_{2}+1}^{C_{2}} \frac{1}{n_{2}^{j}}\right)+\cdots+d_{\left(C_{1}, \ldots, C_{k-1}, 0\right)}\left(\sum_{j=N_{k}+1}^{C_{k}} \frac{1}{n_{1}^{j}}\right) \\
& \leq a_{\left(N_{1}, N_{2}, \ldots, N_{k}\right)}+\frac{\epsilon}{k M} k(\operatorname{dim} \mathcal{D}) \prod_{i=1}^{k} \frac{n_{i}}{n_{i}-1} \\
& =a_{\left(N_{1}, N_{2}, \ldots, N_{k}\right)}+\epsilon \text {. }
\end{aligned}
$$

Consequently, we have

$$
0 \leq a_{\left(C_{1}, C_{2}, \ldots, C_{k}\right)}-a_{\left(N_{1}, N_{2}, \ldots, N_{k}\right)} \leq \epsilon,
$$

which contradicts relation (1.6). Therefore, we must have $L_{i d}=L_{\sigma}$. This completes the proof that the iterated limits $\lim _{q_{\sigma(1)} \rightarrow \infty} \cdots \lim _{q_{\sigma(k)} \rightarrow \infty} a_{\left(q_{1}, \ldots, q_{k}\right)}$ exist and are equal for any permutation $\sigma$ of the set $\{1, \ldots, k\}$.

Now, we show that the net $\left\{a_{\left(q_{1}, \ldots, q_{k}\right)}\right\}_{\left(q_{1}, \ldots, q_{k}\right) \in \mathbb{Z}_{+}^{k}}$ is convergent and

$$
\lim _{\left(q_{1}, \ldots, q_{k}\right) \in \mathbb{Z}_{+}^{k}} a_{\left(q_{1}, \ldots, q_{k}\right)}=L_{i d} .
$$

Assume, by contradiction, that the net $\left\{a_{\left(q_{1}, \ldots, q_{k}\right)}\right\}_{\left(q_{1}, \ldots, q_{k}\right) \in \mathbb{Z}_{+}^{k}}$ is not convergent to $L_{i d}$. Then we can find $\epsilon_{0}>0$ such that for any $\mathbf{q} \in \mathbb{Z}_{+}^{k}$ there exists $\mathbf{p} \in \mathbb{Z}_{+}^{k}$ with $\mathbf{p} \geq \mathbf{q}$ such that

$$
a_{\mathbf{p}} \in\left(-\infty, L_{i d}-3 \epsilon_{0}\right] \cup\left[L_{i d}+3 \epsilon_{0}, \infty\right) .
$$

In particular, for each $\mathbf{q}^{(n)}=(n, \ldots, n) \in \mathbb{Z}_{+}^{k}$ there exists $\mathbf{p}^{(n)} \in \mathbb{Z}_{+}^{k}$ with $\mathbf{p}^{(n)}>\mathbf{q}^{(n)}$ and such that the relation above holds. This implies that at least one of the sets

$$
\Gamma_{-}:=\left\{\mathbf{q} \in \mathbb{Z}_{+}^{k}: a_{\mathbf{q}} \leq L-3 \epsilon_{0}\right\} \quad \text { and } \quad \Gamma_{+}:=\left\{\mathbf{q} \in \mathbb{Z}_{+}^{k}: a_{\mathbf{q}} \geq L+3 \epsilon_{0}\right\}
$$

is cofinal in $\mathbb{Z}_{+}^{k}$. Assume that $\Gamma_{-}$is cofinal in $\mathbb{Z}_{+}^{k}$. Then, using that $n_{i} \geq 2$ for $i \in\{1, \ldots, k\}$, we can find natural numbers $D_{1}, \ldots, D_{k}$ such that $\sum_{j=D_{i}+1}^{\infty} \frac{1}{n_{i}^{j}}<\frac{\epsilon_{0}}{k M}$ for $i \in\{1, \ldots, k\}$, where $M:=$ $(\operatorname{dim} \mathcal{D}) \prod_{i=1}^{k} \frac{n_{i}}{n_{i}-1}$, and such that

$$
a_{\left(D_{1}, \ldots, D_{k}\right)} \leq L_{i d}-3 \epsilon_{0}
$$

Since $L_{i d}=\lim _{q_{1} \rightarrow \infty} \cdots \lim _{q_{k} \rightarrow \infty} a_{\left(q_{1}, \ldots, q_{k}\right)}$, we can choose natural numbers $R_{1}, \ldots, R_{k}$ such that $R_{i}>C_{i}$ for $i \in\{1, \ldots, k\}$ and such that

$$
a_{\left(R_{1}, \ldots, R_{k}\right)} \in\left(L_{i d}-\epsilon_{0}, L+\epsilon_{0}\right) .
$$

Consequently, we deduce that $a_{\left(R_{1}, \ldots, R_{k}\right)}-a_{\left(D_{1}, \ldots, D_{k}\right)} \geq 2 \epsilon_{0}$. On the other hand, as in the first part of the proof, one can show that

$$
a_{\left(R_{1}, \ldots, R_{k}\right)} \leq a_{\left(D_{1}, \ldots, D_{k}\right)}+\epsilon_{0}
$$


which is a contradiction.

Now, assume that $\Gamma_{+}$is a cofinal set in $\mathbb{Z}_{+}^{k}$. Since $n_{i} \geq 2$ and $L_{i d}=\lim _{q_{1} \rightarrow \infty} \cdots \lim _{q_{k} \rightarrow \infty} a_{\left(q_{1}, \ldots, q_{k}\right)}$, we can choose natural numbers $N_{1} \leq N_{2} \leq \ldots \leq N_{k}$ such that $\sum_{j=N_{i}+1}^{\infty} \frac{1}{n_{i}^{j}}<\frac{\epsilon}{k M}$ for any $i \in\{1, \ldots, k\}$, where $M:=(\operatorname{dim} \mathcal{D}) \prod_{i=1}^{k} \frac{n_{i}}{n_{i}-1}$, and such that $\left|a_{\left(N_{1}, \ldots, N_{k}\right)}-L_{i d}\right|<\epsilon$. Taking into account that $\Gamma_{+}$is a cofinal set in $\mathbb{Z}_{+}^{k}$, we can find natural numbers $N_{1}^{\prime}, \ldots, N_{k}^{\prime}$ such that $N_{i}^{\prime} \geq N_{i}$ for any $i \in\{1, \ldots, k\}$ and such that

$$
a_{\left(N_{1}^{\prime}, \ldots, N_{k}^{\prime}\right)} \geq L_{i d}+3 \epsilon_{0}
$$

Consequently, we have $a_{\left(N_{1}^{\prime}, \ldots, N_{k}^{\prime}\right)}-a_{\left(N_{1}, \ldots, N_{k}\right)} \geq 2 \epsilon_{0}$. Once again, as in the first part of the proof, since $\left(N_{1}^{\prime}, \ldots, N_{k}^{\prime}\right) \geq\left(N_{1}, \ldots, N_{k}\right)$ one can show that $a_{\left(N_{1}^{\prime}, \ldots, N_{k}^{\prime}\right)}-a_{\left(N_{1}, \ldots, N_{k}\right)} \leq \epsilon_{0}$, which is a contradiction. This completes the proof.

Let $\mathbf{T}=\left(T_{1}, \ldots, T_{k}\right) \in B(\mathcal{H})^{n_{1}} \times_{c} \cdots \times{ }_{c} B(\mathcal{H})^{n_{k}}$ be a $k$-tuple such that its defect operator defined by $\boldsymbol{\Delta}_{\mathbf{T}}(I):=\left(i d-\Phi_{T_{1}}\right) \cdots\left(i d-\Phi_{T_{k}}\right)(I)$ has finite rank. In this case, we say that $\mathbf{T}$ has finite rank defect or that $\mathbf{T}$ has finite rank. For each $\mathbf{q}=\left(q_{1}, \ldots, q_{k}\right) \in \mathbb{Z}_{+}^{k}$, we define

$$
M_{\mathbf{q}}(\mathbf{T}):=\operatorname{span}\left\{T_{1, \alpha_{1}} \cdots T_{k, \alpha_{k}} h: \alpha_{i} \in \mathbb{F}_{n_{i}}^{+},\left|\alpha_{i}\right| \leq q_{i}, h \in \boldsymbol{\Delta}_{\mathbf{T}}(I)(\mathcal{H})\right\} .
$$

Let $\left\{\mathbf{q}^{(m)}\right\}_{m=1}^{\infty}$ with $\mathbf{q}^{(m)}=\left(q_{1}^{(m)}, \ldots, q_{k}^{(m)}\right)$ be an increasing cofinal sequence in $\mathbb{Z}_{+}^{k}$. Then

$$
M_{\mathbf{q}^{(1)}}(\mathbf{T}) \subseteq M_{\mathbf{q}^{(2)}}(\mathbf{T}) \subseteq \cdots \subseteq M(\mathbf{T}) \quad \text { and } \quad \bigcup_{m=1}^{\infty} M_{\mathbf{q}(m)}(\mathbf{T})=M(\mathbf{T}),
$$

where

$$
M(\mathbf{T}):=\operatorname{span}\left\{T_{1, \alpha_{1}} \cdots T_{k, \alpha_{k}} h: \alpha_{i} \in \mathbb{F}_{n_{i}}^{+}, h \in \boldsymbol{\Delta}_{\mathbf{T}}(I)^{1 / 2}(\mathcal{H})\right\} .
$$

We introduce the Euler characteristic of $\mathbf{T}$ by setting

$$
\chi(\mathbf{T}):=\lim _{m \rightarrow \infty} \frac{\operatorname{dim} M_{\mathbf{q}^{(m)}}(\mathbf{T})}{\prod_{i=1}^{k}\left(1+n_{i}+\cdots+n_{i}^{q_{i}^{(m)}}\right)} .
$$

We remark that the Euler characteristic does not depend on any topology associated with the Hilbert space $\mathcal{H}$ but depends solely on the linear algebra of the linear submanifold $M(\mathbf{T})$ of $\mathcal{H}$.

For each $q_{i} \in\{0,1, \ldots\}$ and $i \in\{1, \ldots, k\}$, let $P_{q_{i}}^{(i)}$ be the orthogonal projection of the full Fock space $F^{2}\left(H_{n_{i}}\right)$ onto the span of all vectors $e_{\alpha_{i}}^{i}$ with $\alpha \in \mathbb{F}_{n_{i}}^{+}$and $\left|\alpha_{i}\right|=q_{i}$. We recall that $P_{\leq\left(q_{1}, \ldots, q_{k}\right)}$ is the orthogonal projection of $\otimes_{i=1}^{k} F^{2}\left(H_{n_{i}}\right)$ onto the span of all vectors of the form $e_{\alpha_{1}}^{1} \otimes \cdots \otimes e_{\alpha_{k}}^{k}$, where $\alpha_{i} \in \mathbb{F}_{n_{i}}^{+},\left|\alpha_{i}\right| \leq q_{i}$. In what follows, we prove the existence of the Euler characteristic associated with each element in the regular polyball and establish several asymptotic formulas.

Theorem 1.2. Let $\mathbf{T}=\left(T_{1}, \ldots, T_{k}\right)$ be in the regular polyball $\mathbf{B}_{\mathbf{n}}(\mathcal{H})$ and have finite rank defect. If $\mathbf{n}:=\left(n_{1}, \ldots, n_{k}\right)$ with $n_{i} \geq 2$, then the Euler characteristic of $\mathbf{T}$ exists and satisfies the asymptotic formulas

$$
\begin{aligned}
\chi(\mathbf{T}) & =\lim _{\mathbf{q}=\left(q_{1}, \ldots, q_{k}\right) \in \mathbb{Z}_{+}^{k}} \frac{\operatorname{dim} M_{\mathbf{q}}(\mathbf{T})}{n_{1}^{q_{1}} \cdots n_{k}^{q_{k}}} \prod_{i=1}^{k}\left(1-\frac{1}{n_{i}}\right) \\
& =\lim _{\mathbf{q}=\left(q_{1}, \ldots, q_{k}\right) \in \mathbb{Z}_{+}^{k}} \frac{\operatorname{rank}\left[\mathbf{K}_{\mathbf{T}}^{*}\left(P_{\leq\left(q_{1}, \ldots, q_{k}\right)} \otimes I\right) \mathbf{K}_{\mathbf{T}}\right]}{\operatorname{rank}\left[P_{\leq\left(q_{1}, \ldots, q_{k}\right)}\right]} \\
& =\lim _{\mathbf{q}=\left(q_{1}, \ldots, q_{k}\right) \in \mathbb{Z}_{+}^{k}} \frac{\operatorname{rank}\left[\left(i d-\Phi_{T_{1}}^{q_{1}+1}\right) \circ \cdots \circ\left(i d-\Phi_{T_{k}}^{q_{k}+1}\right)(I)\right]}{\prod_{i=1}^{k}\left(1+n_{i}+\cdots+n_{i}^{q_{i}}\right)},
\end{aligned}
$$

where $\mathbf{K}_{\mathbf{T}}$ is the Berezin kernel and $\Phi_{T_{1}}, \ldots, \Phi_{T_{k}}$ are the completely positive maps associated with $\mathbf{T}$. 
Proof. Applying Theorem 1.1 when $\mathcal{D}=\boldsymbol{\Delta}_{\mathbf{T}}(I)^{1 / 2}(\mathcal{H})$, we deduce that $\lim _{m \rightarrow \infty} \frac{\operatorname{dim} M_{\mathbf{q}}(m)}{(\mathbf{T})}$ exists and does not depend on the choice of the cofinal sequence $\left\{\mathbf{q}^{(m)}=\left(q_{1}^{(m)}, \ldots, q_{k}^{(m)}\right)\right\}_{m=1}^{\infty}$ in $\mathbb{Z}_{+}^{k}$. Moreover, the limit coincides with

$$
\lim _{\mathbf{q}=\left(q_{1}, \ldots, q_{k}\right) \in \mathbb{Z}_{+}^{k}} \frac{\operatorname{dim} M_{\mathbf{q}}(\mathbf{T})}{n_{1}^{q_{1}} \cdots n_{k}^{q_{k}}}=\lim _{q_{1} \rightarrow \infty} \cdots \lim _{q_{k} \rightarrow \infty} \frac{\operatorname{dim} M_{\mathbf{q}}(\mathbf{T})}{n_{1}^{q_{1}} \cdots n_{k}^{q_{k}}} .
$$

Consequently, the Euler characteristic of $\mathbf{T}$ exists and the first equality in the theorem holds. The noncommutative Berezin kernel associated with $\mathbf{T} \in \mathbf{B}_{\mathbf{n}}(\mathcal{H})$ is the operator

$$
\mathbf{K}_{\mathbf{T}}: \mathcal{H} \rightarrow F^{2}\left(H_{n_{1}}\right) \otimes \cdots \otimes F^{2}\left(H_{n_{k}}\right) \otimes \overline{\boldsymbol{\Delta}_{\mathbf{T}}(I)(\mathcal{H})}
$$

defined by

$$
\mathbf{K}_{\mathbf{T}} h:=\sum_{\beta_{i} \in \mathbb{F}_{n_{i}}^{+}, i=1, \ldots, k} e_{\beta_{1}}^{1} \otimes \cdots \otimes e_{\beta_{k}}^{k} \otimes \boldsymbol{\Delta}_{\mathbf{T}}(I)^{1 / 2} T_{1, \beta_{1}}^{*} \cdots T_{k, \beta_{k}}^{*} h, \quad h \in \mathcal{H} .
$$

It is easy to see that

$$
\mathbf{K}_{\mathbf{T}}^{*}\left(e_{\beta_{1}}^{1} \otimes \cdots \otimes e_{\beta_{k}}^{k} \otimes h\right)=T_{1, \beta_{1}} \cdots T_{k, \beta_{k}} \boldsymbol{\Delta}_{\mathbf{T}}(I)^{1 / 2} h
$$

for any $\beta_{i} \in \mathbb{F}_{n_{i}}^{+}$and $i \in\{1, \ldots, k\}$. This shows that the range of $\mathbf{K}_{\mathbf{T}}^{*}\left(P_{\leq\left(q_{1}, \ldots, q_{k}\right)} \otimes I\right)$ is equal to $M_{\left(q_{1}, \ldots, q_{k}\right)}(\mathbf{T}):=M_{\mathbf{q}}(\mathbf{T})$. Hence, the range of $\mathbf{K}_{\mathbf{T}}^{*}\left(P_{\leq\left(q_{1}, \ldots, q_{k}\right)} \otimes I\right) \mathbf{K}_{\mathbf{T}}$ is equal to $M_{\mathbf{q}}(\mathbf{T})$. Since

$$
\operatorname{rank}\left[P_{\leq\left(q_{1}, \ldots, q_{k}\right)}\right]=\prod_{i=1}^{k}\left(1+n_{i}+\cdots+n_{i}^{q_{i}}\right),
$$

the results above imply

$$
\chi(\mathbf{T})=\lim _{q_{1} \rightarrow \infty} \cdots \lim _{q_{k} \rightarrow \infty} \frac{\operatorname{rank}\left[\mathbf{K}_{\mathbf{T}}^{*}\left(P_{\leq\left(q_{1}, \ldots, q_{k}\right)} \otimes I\right) \mathbf{K}_{\mathbf{T}}\right]}{\operatorname{rank}\left[P_{\leq\left(q_{1}, \ldots, q_{k}\right)}\right]} .
$$

The next step in our proof is to show that

$$
\mathbf{K}_{\mathbf{T}}^{*}\left(P_{\leq\left(q_{1}, \ldots, q_{k}\right)} \otimes I\right) \mathbf{K}_{\mathbf{T}}=\left(i d-\Phi_{T_{1}}^{q_{1}+1}\right) \circ \cdots \circ\left(i d-\Phi_{T_{k}}^{q_{k}+1}\right)(I)
$$

for any $\left(q_{1}, \ldots, q_{k}\right) \in \mathbb{Z}_{+}^{k}$. Let $\mathbf{S}:=\left(\mathbf{S}_{1}, \ldots, \mathbf{S}_{k}\right) \in \mathbf{B}_{\mathbf{n}}\left(\otimes_{i=1}^{k} F^{2}\left(H_{n_{i}}\right)\right)$ with $\mathbf{S}_{i}:=\left(\mathbf{S}_{i, 1}, \ldots, \mathbf{S}_{i, n_{i}}\right)$ be the universal model associated with the abstract polyball $\mathbf{B}_{\mathbf{n}}$. It is easy to see that $\mathbf{S}:=\left(\mathbf{S}_{1}, \ldots, \mathbf{S}_{k}\right)$ is a pure $k$-tuple, i.e. $\Phi_{\mathbf{S}_{i}}^{p}(I) \rightarrow 0$ strongly as $p \rightarrow \infty$, and

$$
\left(i d-\Phi_{\mathbf{S}_{1}}\right) \circ \cdots \circ\left(i d-\Phi_{\mathbf{S}_{k}}\right)(I)=\mathbf{P}_{\mathbb{C}},
$$

where $\mathbf{P}_{\mathbb{C}}$ is the orthogonal projection from $\otimes_{i=1}^{k} F^{2}\left(H_{n_{i}}\right)$ onto $\mathbb{C} 1 \subset \otimes_{i=1}^{k} F^{2}\left(H_{n_{i}}\right)$, where $\mathbb{C} 1$ is identified with $\mathbb{C} 1 \otimes \cdots \otimes \mathbb{C} 1$. Taking into account that $\mathbf{K}_{\mathbf{T}} T_{i, j}^{*}=\left(\mathbf{S}_{i, j}^{*} \otimes I\right) \mathbf{K}_{\mathbf{T}}$ for any $i \in\{1, \ldots, k\}$ and $j \in\left\{1, \ldots, n_{i}\right\}$, we deduce that

$$
\begin{aligned}
\mathbf{K}_{\mathbf{T}}^{*}\left(P_{q_{1}}^{(1)} \otimes \cdots \otimes P_{q_{k}}^{(k)} \otimes I_{\mathcal{H}}\right) \mathbf{K}_{\mathbf{T}} & =\mathbf{K}_{\mathbf{T}}^{*}\left[\left(\Phi_{\mathbf{S}_{1}}^{q_{1}}-\Phi_{\mathbf{S}_{1}}^{q_{1}+1}\right) \circ \cdots \circ\left(\Phi_{\mathbf{S}_{k}}^{q_{k}}-\Phi_{\mathbf{S}_{k}}^{q_{k}+1}\right)(I) \otimes I_{\mathcal{H}}\right] \mathbf{K}_{\mathbf{T}} \\
& =\mathbf{K}_{\mathbf{T}}^{*}\left[\Phi_{\mathbf{S}_{1}}^{q_{1}} \circ \cdots \circ \Phi_{\mathbf{S}_{k}}^{q_{k}} \circ\left(i d-\Phi_{\mathbf{S}_{k}}\right) \circ \cdots \circ\left(i d-\Phi_{\mathbf{S}_{1}}\right)(I) \otimes I_{\mathcal{H}}\right] \mathbf{K}_{\mathbf{T}} \\
& =\Phi_{T_{1}}^{q_{1}} \cdots \cdots \circ \Phi_{T_{k}}^{q_{k}} \circ\left(i d-\Phi_{T_{1}}\right) \circ \cdots \circ\left(i d-\Phi_{T_{k}}\right)\left(\mathbf{K}_{\mathbf{T}}^{*} \mathbf{K}_{\mathbf{T}}\right) .
\end{aligned}
$$

On the other hand, since

$$
\mathbf{K}_{\mathbf{T}}^{*} \mathbf{K}_{\mathbf{T}}=\lim _{q_{k} \rightarrow \infty} \ldots \lim _{q_{1} \rightarrow \infty}\left(i d-\Phi_{T_{k}}^{q_{k}}\right) \circ \cdots \circ\left(i d-\Phi_{T_{1}}^{q_{1}}\right)(I),
$$

where the limits are in the weak operator topology, we can prove that

$$
\left(i d-\Phi_{T_{1}}\right) \circ \cdots \circ\left(i d-\Phi_{T_{k}}\right)\left(\mathbf{K}_{\mathbf{T}}^{*} \mathbf{K}_{\mathbf{T}}\right)=\boldsymbol{\Delta}_{\mathbf{T}}(I) .
$$

Indeed, note that $\left\{\left(i d-\Phi_{T_{k}}^{q_{k}}\right) \circ \cdots \circ\left(i d-\Phi_{T_{1}}^{q_{1}}\right)(I)\right\}_{\mathbf{q}=\left(q_{1}, \ldots, q_{k}\right) \in \mathbb{Z}_{+}^{k}}$ is an increasing sequence of positive operators and

$$
\left(i d-\Phi_{T_{k}}^{q_{k}}\right) \circ \cdots \circ\left(i d-\Phi_{T_{1}}^{q_{1}}\right)(I)=\sum_{s_{k}=0}^{q_{k}-1} \Phi_{T_{k}}^{s_{k}} \circ \cdots \sum_{s_{1}=0}^{q_{1}-1} \Phi_{T_{1}}^{s_{1}} \circ\left(i d-\Phi_{T_{k}}\right) \circ \cdots \circ\left(i d-\Phi_{T_{1}}\right)(I) .
$$


Since $\Phi_{T_{1}}, \ldots, \Phi_{T_{k}}$ are commuting WOT-continuous completely positive linear maps and $\lim _{q_{i} \rightarrow \infty} \Phi_{T_{i}}^{q_{i}}(I)$ exists in the weak operator topology for each $i \in\{1, \ldots, k\}$, we have

$$
\begin{aligned}
\left(i d-\Phi_{T_{1}}\right)\left(\mathbf{K}_{\mathbf{T}}^{*} \mathbf{K}_{\mathbf{T}}\right) & =\lim _{q_{k} \rightarrow \infty} \ldots \lim _{q_{1} \rightarrow \infty}\left(i d-\Phi_{T_{k}}^{q_{k}}\right) \circ \cdots \circ\left(i d-\Phi_{T_{1}}^{q_{1}}\right) \circ\left(i d-\Phi_{T_{1}}\right)(I) \\
& =\lim _{q_{k} \rightarrow \infty} \ldots \lim _{q_{2} \rightarrow \infty}\left(i d-\Phi_{T_{k}}^{q_{k}}\right) \circ \cdots \circ\left(i d-\Phi_{T_{2}}^{q_{2}}\right)\left[\lim _{q_{1} \rightarrow \infty}\left(i d-\Phi_{T_{1}}^{q_{1}}\right) \circ\left(i d-\Phi_{T_{1}}\right)(I)\right] \\
& =\lim _{q_{k} \rightarrow \infty} \ldots \lim _{q_{2} \rightarrow \infty}\left(i d-\Phi_{T_{k}}^{q_{k}}\right) \circ \cdots \circ\left(i d-\Phi_{T_{2}}^{q_{2}}\right) \circ\left(i d-\Phi_{T_{1}}\right)(I) .
\end{aligned}
$$

Applying now $i d-\Phi_{T_{2}}$, a similar reasoning leads to

$\left(i d-\Phi_{T_{2}}\right) \circ\left(i d-\Phi_{T_{1}}\right)\left(\mathbf{K}_{\mathbf{T}}^{*} \mathbf{K}_{\mathbf{T}}\right)=\lim _{q_{k} \rightarrow \infty} \ldots \lim _{q_{3} \rightarrow \infty}\left(i d-\Phi_{T_{k}}^{q_{k}}\right) \circ \cdots \circ\left(i d-\Phi_{T_{3}}^{q_{3}}\right) \circ\left(i d-\Phi_{T_{1}}\right) \circ\left(i d-\Phi_{T_{2}}\right)(I)$.

Continuing this process, we obtain relation (1.9). Combining (1.9) with the relation preceding (1.8), we have

$$
\mathbf{K}_{\mathbf{T}}^{*}\left(P_{q_{1}}^{(1)} \otimes \cdots \otimes P_{q_{k}}^{(k)} \otimes I\right) \mathbf{K}_{\mathbf{T}}=\Phi_{T_{1}}^{q_{1}} \circ \cdots \circ \Phi_{T_{k}}^{q_{k}}\left(\boldsymbol{\Delta}_{\mathbf{T}}(I)\right)
$$

for any $q_{1}, \ldots, q_{k} \in \mathbb{Z}^{+}$, which implies

$$
\begin{aligned}
\mathbf{K}_{\mathbf{T}}^{*}\left(P_{\leq\left(q_{1}, \ldots, q_{k}\right)} \otimes I\right) \mathbf{K}_{\mathbf{T}} & =\sum_{s_{k}=0}^{q_{k}} \Phi_{T_{k}}^{s_{k}} \circ \cdots \sum_{s_{1}=0}^{q_{1}} \Phi_{T_{1}}^{s_{1}} \circ\left(i d-\Phi_{T_{k}}\right) \circ \cdots \circ\left(i d-\Phi_{T_{1}}\right)(I) \\
& =\left(i d-\Phi_{T_{1}}^{q_{1}+1}\right) \circ \cdots \circ\left(i d-\Phi_{T_{k}}^{q_{k}+1}\right)(I)
\end{aligned}
$$

and proves relation (1.7). Now, one can easily complete the proof.

We remark that, in Theorem [1.2, the limit over $\mathbf{q}=\left(q_{1}, \ldots, q_{k}\right) \in \mathbb{Z}_{+}^{k}$ can be replaced by the limit over any increasing cofinal sequence in $\mathbb{Z}_{+}^{k}$, or by any iterated limit $\lim _{q_{\sigma(1)} \rightarrow \infty} \cdots \lim _{q_{\sigma(k)} \rightarrow \infty}$, where $\sigma$ is a permutation of $\{1, \ldots, k\}$.

Corollary 1.3. If $\mathbf{T} \in \mathbf{B}_{\mathbf{n}}(\mathcal{H})$ has finite rank defect, then

$$
0 \leq \operatorname{curv}(\mathbf{T}) \leq \chi(\mathbf{T}) \leq \operatorname{rank}\left[\boldsymbol{\Delta}_{\mathbf{T}}(I)\right]
$$

Proof. According to [24], we have

$$
\operatorname{curv}(\mathbf{T})=\lim _{q_{1} \rightarrow \infty} \cdots \lim _{q_{k} \rightarrow \infty} \frac{\operatorname{trace}\left[\mathbf{K}_{\mathbf{T}}^{*}\left(P_{\leq\left(q_{1}, \ldots, q_{k}\right)} \otimes I_{\mathcal{H}}\right) \mathbf{K}_{\mathbf{T}}\right]}{\operatorname{trace}\left[P_{\leq\left(q_{1}, \ldots, q_{k}\right)}\right]}
$$

Since trace $\left[P_{q_{1}}^{(1)} \otimes \cdots \otimes P_{q_{k}}^{(k)}\right]=\operatorname{rank}\left[P_{q_{1}}^{(1)} \otimes \cdots \otimes P_{q_{k}}^{(k)}\right]$ and $\mathbf{K}_{\mathbf{T}}^{*}\left(P_{\leq\left(q_{1}, \ldots, q_{k}\right)} \otimes I_{\mathcal{H}}\right) \mathbf{K}_{\mathbf{T}}$ is a positive contraction, Theorem 1.2 implies $\operatorname{curv}(\mathbf{T}) \leq \chi(\mathbf{T})$. The inequality $\chi(\mathbf{T}) \leq \operatorname{rank}\left[\boldsymbol{\Delta}_{\mathbf{T}}(I)\right]$ is due to the inequality (1.3), in the particular case when $\mathcal{D}=\boldsymbol{\Delta}_{\mathbf{T}}(I)^{1 / 2}(\mathcal{H})$.

Corollary 1.4. If $\mathbf{T} \in \mathbf{B}_{\mathbf{n}}(\mathcal{H})$ and $\mathbf{T}^{\prime} \in \mathbf{B}_{\mathbf{n}}\left(\mathcal{H}^{\prime}\right)$ have finite rank defects, then $\mathbf{T} \oplus \mathbf{T}^{\prime} \in \mathbf{B}_{\mathbf{n}}\left(\mathcal{H} \oplus \mathcal{H}^{\prime}\right)$ has finite rank defect and

$$
\chi\left(\mathbf{T} \oplus \mathbf{T}^{\prime}\right)=\chi(\mathbf{T})+\chi\left(\mathbf{T}^{\prime}\right) .
$$

If, in addition, $\operatorname{dim} \mathcal{H}^{\prime}<\infty$, then $\chi\left(\mathbf{T} \oplus \mathbf{T}^{\prime}\right)=\chi(\mathbf{T})$.

Given a function $\kappa: \mathbb{N} \rightarrow \mathbb{N}$ and $\mathbf{n}^{(i)}:=\left(n_{1}^{(i)}, \ldots, n_{\kappa(i)}^{(i)}\right) \in \mathbb{N}^{\kappa(i)}$ for $i \in\{1, \ldots, p\}$, we consider the polyball $\mathbf{B}_{\mathbf{n}^{(i)}}\left(\mathcal{H}_{i}\right)$, where $\mathcal{H}_{i}$ is a Hilbert space. Let $\mathbf{X}^{(i)} \in \mathbf{B}_{\mathbf{n}^{(i)}}\left(\mathcal{H}_{i}\right)$ with $\mathbf{X}^{(i)}:=\left(X_{1}^{(i)}, \ldots, X_{\kappa(i)}^{(i)}\right)$ and $X_{r}^{(i)}:=\left(X_{r, 1}^{(i)}, \ldots, X_{r, n_{r}^{(i)}}^{(i)}\right) \in B\left(\mathcal{H}_{i}\right)^{n_{r}^{(i)}}$ for $r \in\{1, \ldots, \kappa(i)\}$. If $\mathbf{X}:=\left(\mathbf{X}^{(1)}, \ldots, \mathbf{X}^{(p)}\right) \in \mathbf{B}_{\mathbf{n}^{(1)}}\left(\mathcal{H}_{1}\right) \times \cdots \times$ $\mathbf{B}_{\mathbf{n}^{(p)}}\left(\mathcal{H}_{p}\right)$, we define the ampliation $\widetilde{\mathbf{X}}$ by setting $\widetilde{\mathbf{X}}:=\left(\widetilde{\mathbf{X}}^{(1)}, \ldots, \widetilde{\mathbf{X}}^{(p)}\right)$, where $\widetilde{\mathbf{X}}^{(i)}:=\left(\widetilde{X}_{1}^{(i)}, \ldots, \widetilde{X}_{\kappa(i)}^{(i)}\right)$ and $\widetilde{X}_{r}^{(i)}:=\left(\widetilde{X}_{r, 1}^{(i)}, \ldots, \widetilde{X}_{r, n_{r}^{(i)}}^{(i)}\right)$ for $r \in\{1, \ldots, \kappa(i)\}$, and

$$
\widetilde{X}_{r, s}^{(i)}:=I_{\mathcal{H}_{1}} \otimes \cdots \otimes I_{\mathcal{H}_{i-1}} \otimes X_{r, s}^{(i)} \otimes I_{\mathcal{H}_{i+1}} \otimes I_{\mathcal{H}_{p}}
$$

for all $i \in\{1, \ldots, p\}, r \in\{1, \ldots, \kappa(i)\}$, and $s \in\left\{1, \ldots, n_{r}^{(i)}\right\}$. 
Theorem 1.5. Let $\mathbf{X}:=\left(\mathbf{X}^{(1)}, \ldots, \mathbf{X}^{(p)}\right) \in \mathbf{B}_{\mathbf{n}^{(1)}}\left(\mathcal{H}_{1}\right) \times \cdots \times \mathbf{B}_{\mathbf{n}^{(p)}}\left(\mathcal{H}_{p}\right)$ be such that each $\mathbf{X}^{(i)}$ has finite rank defect. Then the ampliation $\widetilde{\mathbf{X}}$ is in the regular polyball $\mathbf{B}_{\left(\mathbf{n}^{(1)}, \ldots, \mathbf{n}^{(p)}\right)}\left(\mathcal{H}_{1} \otimes \cdots \otimes \mathcal{H}_{p}\right)$, has finite rank defect, and the Euler characteristic satisfies the relation

$$
\chi(\widetilde{\mathbf{X}})=\prod_{i=1}^{p} \chi\left(\mathbf{X}^{(i)}\right) .
$$

Proof. For each $\mathbf{m}^{(i)} \in \mathbb{Z}_{+}^{\kappa(i)}$ with $0 \leq \mathbf{m}^{(i)} \leq(1, \ldots, 1)$ and $i \in\{1, \ldots, p\}$, we have

$$
\boldsymbol{\Delta}_{\widetilde{\mathbf{X}}}^{\left(\mathbf{m}^{(1)}, \ldots, \mathbf{m}^{(p)}\right)}\left(I_{\mathcal{H}_{1} \otimes \cdots \otimes \mathcal{H}_{p}}\right)=\boldsymbol{\Delta}_{\mathbf{X}^{(1)}}^{\mathbf{m}^{(1)}}\left(I_{\mathcal{H}_{1}}\right) \otimes \cdots \otimes \boldsymbol{\Delta}_{\mathbf{X}^{(p)}}^{\mathbf{m}^{(p)}}\left(I_{\mathcal{H}_{p}}\right) \geq 0 .
$$

Consequently, $\widetilde{\mathbf{X}}$ is in the regular polyball $\mathbf{B}_{\left(\mathbf{n}^{(1)}, \ldots, \mathbf{n}^{(p)}\right)}\left(\mathcal{H}_{1} \otimes \cdots \otimes \mathcal{H}_{p}\right)$ and

$$
\operatorname{rank}\left[\boldsymbol{\Delta}_{\widetilde{\mathbf{X}}}\left(I_{\mathcal{H}_{1} \otimes \cdots \otimes \mathcal{H}_{p}}\right)\right]=\operatorname{rank}\left[\boldsymbol{\Delta}_{\mathbf{X}^{(1)}}\left(I_{\mathcal{H}_{1}}\right)\right] \cdots \operatorname{rank}\left[\boldsymbol{\Delta}_{\mathbf{X}^{(p)}}\left(I_{\mathcal{H}_{p}}\right)\right]<\infty .
$$

Let $\mathbf{q}^{(i)}:=\left(q_{1}^{(i)}, \ldots, q_{\kappa(i)}^{(i)}\right) \in \mathbb{Z}_{+}^{\kappa(i)}$ for $i \in\{1, \ldots, p\}$. According to Theorem 1.2, the Euler characteristic $\chi(\widetilde{\mathbf{X}})$ is equal

$$
\lim \frac{\operatorname{rank}\left[\left(i d-\Phi_{X_{1}^{(1)}}^{q_{q}^{(1)}+1}\right) \circ \cdots \circ\left(i d-\Phi_{X_{\kappa(1)}^{(1)}}^{q_{\kappa^{(1)}}^{(1)}}\right)\left(I_{\mathcal{H}_{1}}\right) \otimes \cdots \otimes\left(i d-\Phi_{X_{1}^{(p)}}^{q_{q}^{(p)}+1}\right) \circ \cdots \circ\left(i d-\Phi_{X_{\kappa(p)}^{(p)}}^{q_{q^{(p)}}^{(p)}+1}\right)\left(I_{\mathcal{H}_{p}}\right)\right]}{\prod_{r=1}^{\kappa(1)}\left(1+n_{r}^{(1)}+\cdots+\left(n_{r}^{(1)}\right)^{q_{r}^{(1)}}\right) \cdots \prod_{r=1}^{\kappa(p)}\left(1+n_{r}^{(p)}+\cdots+\left(n_{r}^{(p)}\right)^{q_{r}^{(p)}}\right)},
$$

where the limit is taken over $\mathbf{q}^{(1)} \in \mathbb{Z}_{+}^{\kappa(1)}, \ldots, \mathbf{q}^{(p)} \in \mathbb{Z}_{+}^{\kappa(p)}$, which is equal to to the product

$$
\prod_{i=1}^{p} \lim _{\mathbf{q}^{(i)} \in \mathbb{Z}_{+}^{\kappa(i)}} \frac{\operatorname{rank}\left[\left(i d-\Phi_{X_{1}^{(i)}}^{q_{1}^{(i)}+1}\right) \circ \cdots \circ\left(i d-\Phi_{X_{\kappa(i)}^{(i)}}^{q_{\kappa(i)}^{(i)}}\right)\left(I_{\mathcal{H}_{i}}\right)\right]}{\prod_{r=1}^{\kappa(i)}\left(1+n_{r}^{(i)}+\cdots+\left(n_{r}^{(i)}\right)_{r}^{(i)}\right)} .
$$

Due to Theorem 1.2, the latter product is equal to $\prod_{i=1}^{p} \chi\left(\mathbf{X}^{(i)}\right)$. The proof is complete.

\section{InVAriant subspaces, Euler characteristic, AND CLASSIFICATION}

In this section, we give a characterization of the invariant subspaces of the tensor product $\otimes_{i=1}^{k} F^{2}\left(H_{n_{i}}\right)$ with positive defect operators. We show that the Euler characteristic completely classifies the finite rank Beurling type invariant subspaces of $\otimes_{i=1}^{k} F^{2}\left(H_{n_{i}}\right)$ and prove some of its basic properties, including the fact that its range is the interval $[0, \infty)$.

Let $\mathbf{S}:=\left(\mathbf{S}_{1}, \ldots, \mathbf{S}_{k}\right)$, where $\mathbf{S}_{i}:=\left(\mathbf{S}_{i, 1}, \ldots, \mathbf{S}_{i, n_{i}}\right)$, be the universal model of the abstract polyball $\mathbf{B}_{\mathbf{n}}$, and let $\mathcal{H}$ be a Hilbert space. We say that $\mathcal{M}$ is an invariant subspace of $\otimes_{i=1}^{k} F^{2}\left(H_{n_{i}}\right) \otimes \mathcal{H}$ or that $\mathcal{M}$ is invariant under $\mathbf{S} \otimes I_{\mathcal{H}}$ if it is invariant under each operator $\mathbf{S}_{i, j} \otimes I_{\mathcal{H}}$ for $i \in\{1, \ldots, k\}$ and $j \in\left\{1, \ldots, n_{j}\right\}$.

Definition 2.1. Given two invariant subspaces $\mathcal{M}$ and $\mathcal{N}$ under $\mathbf{S} \otimes I_{\mathcal{H}}$, we say that they are unitarily equivalent if there is a unitary operator $U: \mathcal{M} \rightarrow \mathcal{N}$ such that $\left.U\left(\mathbf{S}_{i, j} \otimes I_{\mathcal{H}}\right)\right|_{\mathcal{M}}=\left.\left(\mathbf{S}_{i, j} \otimes I_{\mathcal{H}}\right)\right|_{\mathcal{N}} U$ for any $i \in\{1, \ldots, k\}$ and $j \in\left\{1, \ldots, n_{i}\right\}$.

We define the right creation operator $R_{i, j}: F^{2}\left(H_{n_{i}}\right) \rightarrow F^{2}\left(H_{n_{i}}\right)$ by setting

$$
R_{i, j} e_{\alpha}^{i}:=e_{\alpha g_{j}}^{i}, \quad \alpha \in \mathbb{F}_{n_{i}}^{+},
$$

and the operator $\mathbf{R}_{i, j}$ acting on the tensor Hilbert space $F^{2}\left(H_{n_{1}}\right) \otimes \cdots \otimes F^{2}\left(H_{n_{k}}\right)$. We also denote $\mathbf{R}:=\left(\mathbf{R}_{1}, \ldots, \mathbf{R}_{k}\right)$, where $\mathbf{R}_{i}:=\left(\mathbf{R}_{i, 1}, \ldots, \mathbf{R}_{i, n_{i}}\right)$. Let $\varphi=\sum_{\beta_{i} \in \mathbb{F}_{n_{i}}^{+}} c_{\beta_{1}, \ldots, \beta_{k}} e_{\beta_{1}}^{1} \otimes \cdots \otimes e_{\beta_{k}}^{k}$ be in $\otimes_{i=1}^{k} F^{2}\left(H_{n_{i}}\right)$ and consider the formal power series $\widetilde{\varphi}\left(\mathbf{R}_{i, j}\right):=\sum_{\beta_{i} \in \mathbb{F}_{n_{i}}^{+}} c_{\beta_{1}, \ldots, \beta_{k}} \mathbf{R}_{1, \widetilde{\beta}_{1}} \cdots \mathbf{R}_{k, \widetilde{\beta}_{k}}$, where $\widetilde{\beta}_{i}=g_{j_{p}}^{i} \cdots g_{j_{1}}^{i}$ denotes the reverse of $\beta_{i}=g_{j_{1}}^{i} \cdots g_{j_{p}}^{i} \in \mathbb{F}_{n_{i}}^{+}$. Note that $\widetilde{\varphi}\left(\mathbf{R}_{i, j}\right)\left(e_{\gamma_{1}}^{1} \otimes \cdots \otimes e_{\gamma_{k}}^{k}\right)$ is in $\otimes_{i=1}^{k} F^{2}\left(H_{n_{i}}\right)$. We say that $\varphi$ is a right multiplier of $\otimes_{i=1}^{k} F^{2}\left(H_{n_{i}}\right)$ if

$$
\sup _{p \in \mathcal{P},\|p\| \leq 1}\left\|\widetilde{\varphi}\left(\mathbf{R}_{i, j}\right) p\right\|<\infty,
$$


where $\mathcal{P}$ is the set of all polynomials $\sum a_{\alpha_{1}, \ldots, \alpha_{k}} e_{\alpha_{1}}^{1} \otimes \cdots \otimes e_{\alpha_{k}}^{k}$ in $\otimes_{i=1}^{k} F^{2}\left(H_{n_{i}}\right)$. In this case, there is a unique bounded operator acting on $\otimes_{i=1}^{k} F^{2}\left(H_{n_{i}}\right)$, denoted also by $\widetilde{\varphi}\left(\mathbf{R}_{i, j}\right)$, such that

$$
\widetilde{\varphi}\left(\mathbf{R}_{i, j}\right) p=\sum_{\beta_{i} \in \mathbb{F}_{n_{i}}^{+}} c_{\beta_{1}, \ldots, \beta_{k}} \mathbf{R}_{1, \widetilde{\beta}_{1}} \cdots \mathbf{R}_{k, \widetilde{\beta}_{k}} p, \quad p \in \mathcal{P} .
$$

The set of all operators $\widetilde{\varphi}\left(\mathbf{R}_{i, j}\right)$ satisfying the conditions above is a Banach algebra denoted by $R^{\infty}\left(\mathbf{B}_{\mathbf{n}}\right)$. We proved in 22] that $F^{\infty}\left(\mathbf{B}_{\mathbf{n}}\right)^{\prime}=R^{\infty}\left(\mathbf{B}_{\mathbf{n}}\right)$ and $F^{\infty}\left(\mathbf{B}_{\mathbf{n}}\right)^{\prime \prime}=F^{\infty}\left(\mathbf{B}_{\mathbf{n}}\right)$, where 'stands for the commutant.

According to Theorem 5.1 from [22], if a subspace $\mathcal{M} \subset \otimes_{i=1}^{k} F^{2}\left(H_{n_{i}}\right) \otimes \mathcal{H}$ is co-invariant under each operator $\mathbf{S}_{i, j} \otimes I_{\mathcal{H}}$, then

$$
\overline{\operatorname{span}}\left\{\left(\mathbf{S}_{1, \beta_{1}} \cdots \mathbf{S}_{k, \beta_{k}} \otimes I_{\mathcal{H}}\right) \mathcal{M}: \beta_{1} \in \mathbb{F}_{n_{1}}^{+}, \ldots, \beta_{k} \in \mathbb{F}_{n_{k}}^{+}\right\}=\otimes_{i=1}^{k} F^{2}\left(H_{n_{i}}\right) \otimes \mathcal{E},
$$

where $\mathcal{E}:=\left(\mathbf{P}_{\mathbb{C}} \otimes I_{\mathcal{H}}\right)(\mathcal{M})$. Consequently, a subspace $\mathcal{R} \subseteq \otimes_{i=1}^{k} F^{2}\left(H_{n_{i}}\right) \otimes \mathcal{H}$ is reducing under $\mathbf{S} \otimes I_{\mathcal{H}}$ if and only if there exists a subspace $\mathcal{G} \subseteq \mathcal{H}$ such that $\mathcal{R}=\otimes_{i=1}^{k} F^{2}\left(H_{n_{i}}\right) \otimes \mathcal{G}$.

It is well known [26] that the lattice of the invariant subspaces for the Hardy space $H^{2}\left(\mathbb{D}^{k}\right)$ is very complicated and contains many invariant subspaces which are not of Beurling type. The same complicated situation occurs in the case of the tensor product $\otimes_{i=1}^{k} F^{2}\left(H_{n_{i}}\right)$. Following the classical case 44, we say that $\mathcal{M}$ is a Beurling type invariant subspace for $\mathbf{S} \otimes I_{\mathcal{H}}$ if there is an inner multi-analytic operator $\Psi: \otimes_{i=1}^{k} F^{2}\left(H_{n_{i}}\right) \otimes \mathcal{E} \rightarrow \otimes_{i=1}^{k} F^{2}\left(H_{n_{i}}\right) \otimes \mathcal{H}$ with respect to $\mathbf{S}$, where $\mathcal{E}$ is a Hilbert space, such that $\mathcal{M}=\Psi\left[\otimes_{i=1}^{k} F^{2}\left(H_{n_{i}}\right) \otimes \mathcal{E}\right]$. In this case, $\Psi$ can be chosen to be isometric. We recall that $\Psi$ is multianalytic if $\Psi\left(\mathbf{S}_{i, j} \otimes I_{\mathcal{H}}\right)=\left(\mathbf{S}_{i, j} \otimes I_{\mathcal{E}}\right) \Psi$ for all $i, j$. More about multi-analytic operators on Fock spaces can be found in [15] and [16]. In [22], we proved that $\mathcal{M}$ is a Beurling type invariant subspace for $\mathbf{S} \otimes I_{\mathcal{H}}$ if and only if

$$
\left(i d-\Phi_{\mathbf{S}_{1} \otimes I_{\mathcal{H}}}\right) \circ \cdots \circ\left(i d-\Phi_{\mathbf{S}_{k} \otimes I_{\mathcal{H}}}\right)\left(P_{\mathcal{M}}\right) \geq 0,
$$

where $P_{\mathcal{M}}$ is the orthogonal projection onto $\mathcal{M}$. We introduce the defect operator of $\mathcal{M}$ be setting $\Delta_{\mathcal{M}}:=\Delta_{\mathbf{S} \otimes I_{\mathcal{H}}}\left(P_{\mathcal{M}}\right)$.

In what follows, we present a more direct and more transparent proof for the characterization of the invariant subspaces of $\otimes_{i=1}^{k} F^{2}\left(H_{n_{i}}\right)$ with positive defect operator, which complements the corresponding result from 22] (see Corollary 5.3).

Theorem 2.2. A subspace $\mathcal{M} \subseteq \otimes_{i=1}^{k} F^{2}\left(H_{n_{i}}\right), n_{i} \geq 1$, is invariant under the universal model $\mathbf{S}$ and has positive defect operator $\Delta_{\mathcal{M}}$ if and only if there is a sequence $\left\{\psi_{s}\right\}_{s=1}^{N}$, where $N \in \mathbb{N}$ or $N=\infty$, of right multipliers of $\otimes_{i=1}^{k} F^{2}\left(H_{n_{i}}\right)$ such that $\left\{\widetilde{\psi}_{s}\left(\mathbf{R}_{i, j}\right)\right\}_{s=1}^{N}$ are isometries with orthogonal ranges and

$$
P_{\mathcal{M}}=\sum_{s=1}^{N} \widetilde{\psi}_{s}\left(\mathbf{R}_{i, j}\right) \widetilde{\psi}_{s}\left(\mathbf{R}_{i, j}\right)^{*}
$$

where the convergence is in the strong operator topology. The sequence $\left\{\psi_{s}\right\}_{s=1}^{N}$ is uniquely determined up to a unitary equivalence.

In addition, we can choose the sequence $\left\{\psi_{s}\right\}_{s=1}^{N}$ such that each $\psi_{s}$ is in the range of $\Delta_{\mathcal{M}}$. We also have $N=\operatorname{rank}\left(\Delta_{\mathcal{M}}^{1 / 2}\right)$ and

$$
\Delta_{\mathcal{M}} \xi=\sum_{t=1}^{N}\left\langle\xi, \psi_{s}\right\rangle \psi_{s}, \quad \xi \in \otimes_{i=1}^{k} F^{2}\left(H_{n_{i}}\right) .
$$

Proof. Assume that $\mathcal{M} \subseteq \otimes_{i=1}^{k} F^{2}\left(H_{n_{i}}\right)$ is an invariant subspace under $\mathbf{S}$ and $\Delta_{\mathcal{M}}:=\boldsymbol{\Delta}_{\mathbf{S}}\left(P_{\mathcal{M}}\right) \geq 0$. Let $\mathbf{A}=\left(A_{1}, \ldots, A_{k}\right)$ with $A_{i}:=\left(\left.\mathbf{S}_{i, 1}\right|_{\mathcal{M}}, \ldots,\left.\mathbf{S}_{i, n_{i}}\right|_{\mathcal{M}}\right)$ and note that $\mathbf{A}$ is a pure element in $\mathbf{B}_{\mathbf{n}}(\mathcal{M})$. The noncommutative Berezin kernel associated with $\mathbf{A}$,

$$
\mathbf{K}_{\mathbf{A}}: \mathcal{M} \rightarrow\left(\otimes_{i=1}^{k} F^{2}\left(H_{n_{i}}\right)\right) \otimes \overline{\boldsymbol{\Delta}_{\mathbf{A}}\left(I_{\mathcal{M}}\right)^{1 / 2}(\mathcal{M})},
$$

is an isometry. Note that $\Delta_{\mathcal{M}}(\mathcal{M}) \subseteq \mathcal{M}$ and $\left.\Delta_{\mathcal{M}}\right|_{\mathcal{M}}=\boldsymbol{\Delta}_{\mathbf{A}}\left(I_{\mathcal{M}}\right)$. Consequently, we have $\Delta_{\mathcal{M}}\left(\mathcal{M}^{\perp}\right) \subseteq$ $\mathcal{M}^{\perp}$ and $\boldsymbol{\Delta}_{\mathbf{A}}\left(I_{\mathcal{M}}\right)^{1 / 2}(\mathcal{M})=\Delta_{\mathcal{M}}^{1 / 2}\left(\otimes_{i=1}^{k} F^{2}\left(H_{n_{i}}\right)\right)$. Consider the extended Berezin kernel

$$
\widehat{\mathbf{K}}_{\mathbf{A}}: \otimes_{i=1}^{k} F^{2}\left(H_{n_{i}}\right) \rightarrow\left(\otimes_{i=1}^{k} F^{2}\left(H_{n_{i}}\right)\right) \otimes \overline{\Delta_{\mathcal{M}}^{1 / 2}\left(\otimes_{i=1}^{k} F^{2}\left(H_{n_{i}}\right)\right)}
$$


defined by $\left.\widehat{\mathbf{K}}_{\mathbf{A}}\right|_{\mathcal{M}}:=\mathbf{K}_{\mathbf{A}}$ and $\left.\widehat{\mathbf{K}}_{\mathbf{A}}\right|_{\mathcal{M}^{\perp}}:=0$. Let $\left\{\chi_{s}\right\}_{s=1}^{N}$ be an orthonormal basis for the defect space $\overline{\Delta_{\mathcal{M}}^{1 / 2}\left(\otimes_{i=1}^{k} F^{2}\left(H_{n_{i}}\right)\right)}$ and set

$$
\psi_{s}:=\widehat{\mathbf{K}}_{\mathbf{A}}^{*}\left(1 \otimes \chi_{s}\right)=\mathbf{K}_{\mathbf{A}}^{*}\left(1 \otimes \chi_{s}\right)=\Delta_{\mathbf{A}}\left(I_{\mathcal{M}}\right)^{1 / 2} \chi_{s}=\Delta_{\mathcal{M}}^{1 / 2} \chi_{s} .
$$

If $p=\sum a_{\alpha_{1}, \ldots, \alpha_{k}} e_{\alpha_{1}}^{1} \otimes \cdots \otimes e_{\alpha_{k}}^{k}$ is any polynomial in $\otimes_{i=1}^{k} F^{2}\left(H_{n_{i}}\right)$, we have

$$
\mathbf{K}_{\mathbf{A}}^{*}\left(p \otimes \chi_{s}\right)=p\left(\left.\mathbf{S}_{i, j}\right|_{\mathcal{M}}\right) \Delta_{\mathcal{M}}^{1 / 2} \chi_{s}=p\left(\mathbf{S}_{i, j}\right) \psi_{s}=p \psi_{s}=\widetilde{\psi}_{s}\left(\mathbf{R}_{i, j}\right) p
$$

Hence,

$$
\left\|\widetilde{\psi}_{s}\left(\mathbf{R}_{i, j}\right) p\right\|=\left\|\mathbf{K}_{\mathbf{A}}^{*}\left(p \otimes \chi_{s}\right)\right\| \leq\|p\| .
$$

Since the polynomials are dense in $\otimes_{i=1}^{k} F^{2}\left(H_{n_{i}}\right)$, we deduce that $\psi_{s}$ is a right multiplier of $\otimes_{i=1}^{k} F^{2}\left(H_{n_{i}}\right)$. For each $s \in\{1, \ldots, N\}$, we define the linear operator $\Gamma_{s}: \otimes_{i=1}^{k} F^{2}\left(H_{n_{i}}\right) \rightarrow \otimes_{i=1}^{k} F^{2}\left(H_{n_{i}}\right)$ by setting

$$
\left\langle\Gamma_{s} f, g\right\rangle:=\left\langle\widehat{\mathbf{K}}_{\mathbf{A}} f, g \otimes \chi_{s}\right\rangle, \quad f, g \in \otimes_{i=1}^{k} F^{2}\left(H_{n_{i}}\right) .
$$

Note that

$$
\begin{aligned}
\sum_{s=1}^{N}\left\|\Gamma_{s} f\right\|^{2} & =\sum_{s=1}^{N} \sum_{\alpha_{i} \in \mathbb{F}_{n_{i}}^{+}}\left|\left\langle\Gamma_{s} f, e_{\alpha_{1}}^{1} \otimes \cdots \otimes e_{\alpha_{k}}^{k}\right\rangle\right|^{2}=\sum_{s=1}^{N} \sum_{\alpha_{i} \in \mathbb{F}_{n_{i}}^{+}}\left|\left\langle\widehat{\mathbf{K}}_{\mathbf{A}} f,\left(e_{\alpha_{1}}^{1} \otimes \cdots \otimes e_{\alpha_{k}}^{k}\right) \otimes \chi_{s}\right\rangle\right|^{2} \\
& =\left\|\widehat{\mathbf{K}}_{\mathbf{A}} f\right\|^{2} \leq\left\|\mathbf{K}_{\mathbf{A}} f\right\|^{2}=\|f\|^{2}
\end{aligned}
$$

for any $f \in \otimes_{i=1}^{k} F^{2}\left(H_{n_{i}}\right)$. Consequently, $\sum_{s=1}^{N} \Gamma_{s}^{*} \Gamma_{s}$ is convergent in the strong operator topology and

$$
\sum_{s=1}^{N} \Gamma_{s}^{*} \Gamma_{s}=\widehat{\mathbf{K}}_{\mathbf{A}}^{*} \widehat{\mathbf{K}}_{\mathbf{A}}=\left[\begin{array}{cc}
\mathbf{K}_{\mathbf{A}}^{*} \mathbf{K}_{\mathbf{A}} & 0 \\
0 & 0
\end{array}\right]=\left[\begin{array}{cc}
I_{\mathcal{M}} & 0 \\
0 & 0
\end{array}\right]=P_{\mathcal{M}}
$$

Note also that

$$
\left\langle\Gamma_{s} f, p\right\rangle=\left\langle f, \mathbf{K}_{\mathbf{A}}^{*}\left(p \otimes \chi_{s}\right)\right\rangle=\left\langle f, \widetilde{\psi}_{s}\left(\mathbf{R}_{i, j}\right) p\right\rangle=\left\langle\widetilde{\psi}_{s}\left(\mathbf{R}_{i, j}\right)^{*} f, p\right\rangle .
$$

Since the polynomials are dense in $\otimes_{i=1}^{k} F^{2}\left(H_{n_{i}}\right)$, we deduce that $\Gamma_{s}=\widetilde{\psi}_{s}\left(\mathbf{R}_{i, j}\right)^{*}$ and, consequently, $\sum_{s=1}^{N} \widetilde{\psi}_{s}\left(\mathbf{R}_{i, j}\right) \widetilde{\psi}_{s}\left(\mathbf{R}_{i, j}\right)^{*}=P_{\mathcal{M}}$, where the convergence is in the strong operator topology. On the other hand, since $\Phi_{\mathbf{S}_{i}}$ is WOT-continuous, so is the defect map $\boldsymbol{\Delta}_{\mathbf{S}}:=\left(i d-\Phi_{\mathbf{S}_{1}}\right) \circ \cdots \circ\left(i d-\Phi_{\mathbf{S}_{k}}\right)$. Consequently,

$$
\begin{aligned}
\Delta_{\mathcal{M}} & =\Delta_{\mathbf{S}}\left(P_{\mathcal{M}}\right)=\sum_{s=1}^{N} \widetilde{\psi}_{s}\left(\mathbf{R}_{i, j}\right) \boldsymbol{\Delta}_{\mathbf{S}}(I) \widetilde{\psi}_{s}\left(\mathbf{R}_{i, j}\right)^{*} \\
& =\sum_{s=1}^{N} \widetilde{\psi}_{s}\left(\mathbf{R}_{i, j}\right) P_{\mathbb{C}} \widetilde{\psi}_{s}\left(\mathbf{R}_{i, j}\right)^{*} .
\end{aligned}
$$

Hence, we deduce that

$$
\Delta_{\mathcal{M}} \xi=\sum_{s=1}^{N} \widetilde{\psi}_{s}\left(\mathbf{R}_{i, j}\right)\left\langle\widetilde{\psi}_{s}\left(\mathbf{R}_{i, j}\right)^{*} \xi, 1\right\rangle=\sum_{t=1}^{N}\left\langle\xi, \psi_{s}\right\rangle \psi_{s}, \quad \xi \in \otimes_{i=1}^{k} F^{2}\left(H_{n_{i}}\right) .
$$

Let $\Psi:\left(\otimes_{i=1}^{k} F^{2}\left(H_{n_{i}}\right)\right) \otimes \mathbb{C}^{N} \rightarrow \otimes_{i=1}^{k} F^{2}\left(H_{n_{i}}\right)$ be the bounded operator having the $1 \times N$ matrix representation

$$
\left[\widetilde{\psi}_{1}\left(\mathbf{R}_{i, j}\right), \widetilde{\psi}_{2}\left(\mathbf{R}_{i, j}\right), \ldots\right],
$$

where $\mathbb{C}^{\infty}$ stands for $\ell^{2}(\mathbb{N})$. Note that $\Psi$ is a multi-analytic operator with respect to the universal model $\mathbf{S}$ and $\Psi \Psi^{*}=P_{\mathcal{M}}$. Therefore, $\Psi$ is a partial isometry. Since $\mathbf{S}_{i, j}$ are isometries, the initial space of $\Psi$, i.e.

$$
\Psi^{*}\left(\otimes_{i=1}^{k} F^{2}\left(H_{n_{i}}\right)\right)=\left\{x \in\left(\otimes_{i=1}^{k} F^{2}\left(H_{n_{i}}\right)\right) \otimes \mathbb{C}^{N}:\|\Psi x\|=\|x\|\right\}
$$

is reducing under each operator $\mathbf{S}_{i, j} \otimes I_{\mathbb{C}^{N}}$. Consequently, since $\boldsymbol{\Delta}_{\mathbf{S}}(I)=P_{\mathbb{C}}$, to prove that $\Psi$ is an isometry, it is enough to show that

$$
\mathbb{C}=P_{\mathbb{C}} \widetilde{\psi}_{s}\left(\mathbf{R}_{i, j}\right)^{*}\left(\otimes_{i=1}^{k} F^{2}\left(H_{n_{i}}\right)\right) \quad \text { for each } s \in\{1, \ldots, N\} .
$$


The latter equality is true since

$$
P_{\mathbb{C}} \widetilde{\psi}_{s}\left(\mathbf{R}_{i, j}\right)^{*}\left(\psi_{s}\right)=\left\langle\widetilde{\psi}_{s}\left(\mathbf{R}_{i, j}\right)^{*}\left(\psi_{s}\right), 1\right\rangle=\left\|\psi_{s}\right\|^{2}=1
$$

Therefore, $\Psi$ is an isometry.

To prove the converse of the theorem, assume that there is a sequence $\left\{\psi_{s}\right\}_{s=1}^{N}$ of right multipliers of $\otimes_{i=1}^{k} F^{2}\left(H_{n_{i}}\right)$ such that $\left\{\widetilde{\psi}_{s}\left(\mathbf{R}_{i, j}\right)\right\}_{s=1}^{N}$ are isometries with orthogonal ranges and

$$
P_{\mathcal{M}}=\sum_{s=1}^{N} \widetilde{\psi}_{s}\left(\mathbf{R}_{i, j}\right) \widetilde{\psi}_{s}\left(\mathbf{R}_{i, j}\right)^{*}
$$

Since $\mathbf{S}_{i, j}$ commutes with $\mathbf{R}_{r, q}$ for any $i, r \in\{1, \ldots, k\}, j \in\left\{1, \ldots, n_{i}\right\}$, and $q \in\left\{1, \ldots, n_{r}\right\}$, and $\left\{\widetilde{\psi}_{s}\left(\mathbf{R}_{i, j}\right)\right\}_{s=1}^{N}$ are isometries with orthogonal ranges, we deduce that $P_{\mathcal{M}} \mathbf{S}_{i, j} P_{\mathcal{M}}=\mathbf{S}_{i, j} P_{\mathcal{M}}$. Therefore, $\mathcal{M}$ is an invariant subspace under the universal model $\mathbf{S}$. Moreover, we have

$$
\Delta_{\mathcal{M}}=\left(i d-\Phi_{\mathbf{S}_{1}}\right) \circ \cdots \circ\left(i d-\Phi_{\mathbf{S}_{k}}\right)\left(P_{\mathcal{M}}\right)=\sum_{s=1}^{N} \widetilde{\psi}_{s}\left(\mathbf{R}_{i, j}\right) \boldsymbol{\Delta}_{\mathbf{S}}(I) \widetilde{\psi}_{s}\left(\mathbf{R}_{i, j}\right)^{*} \geq 0 .
$$

Now, we prove that the sequence $\left\{\psi_{s}\right\}_{s=1}^{N}$ is uniquely determined up to a unitary equivalence. Let $\left\{\psi_{s}^{\prime}\right\}_{s=1}^{N^{\prime}}$ be another sequence of right multipliers of $\otimes_{i=1}^{k} F^{2}\left(H_{n_{i}}\right)$ such that $\left\{\widetilde{\psi}_{s}^{\prime}\left(\mathbf{R}_{i, j}\right)\right\}_{s=1}^{N^{\prime}}$ are isometries with orthogonal ranges and

$$
P_{\mathcal{M}}=\sum_{s=1}^{N^{\prime}} \widetilde{\psi}_{s}^{\prime}\left(\mathbf{R}_{i, j}\right) \widetilde{\psi}_{s}^{\prime}\left(\mathbf{R}_{i, j}\right)^{*}
$$

As above, we have

$$
\Delta_{\mathcal{M}} \xi=\sum_{t=1}^{N}\left\langle\xi, \psi_{s}\right\rangle \psi_{s}=\sum_{t=1}^{N^{\prime}}\left\langle\xi, \psi_{s}^{\prime}\right\rangle \psi_{s}^{\prime}, \quad \xi \in \otimes_{i=1}^{k} F^{2}\left(H_{n_{i}}\right)
$$

Consequently, if $\Delta_{\mathcal{M}}$ has finite rank then $\left\{\psi_{s}\right\}_{s=1}^{N}$ and $\left\{\psi_{s}^{\prime}\right\}_{s=1}^{N^{\prime}}$ are orthonormal bases for the range of $\Delta_{\mathcal{M}}$, thus $N=N^{\prime} \in \mathbb{N}$. If $\Delta_{\mathcal{M}}$ does not have finite rank, similar arguments, show that $N=N^{\prime}=\infty$. Now, note that

$$
\mathcal{M}=\bigoplus_{s=1}^{N}\left(\otimes_{i=1}^{k} F^{2}\left(H_{n_{i}}\right)\right) \psi_{s}=\bigoplus_{s=1}^{N}\left(\otimes_{i=1}^{k} F^{2}\left(H_{n_{i}}\right)\right) \psi_{s}^{\prime}
$$

and $\left\{\left(e_{\alpha_{1}}^{1} \otimes \cdots \otimes e_{\alpha_{k}}^{k}\right) \psi_{s}: \alpha_{i} \in \mathbb{F}_{n_{i}}^{+}, s \in\{1, \ldots, N\}\right\}$ and $\left\{\left(e_{\alpha_{1}}^{1} \otimes \cdots \otimes e_{\alpha_{k}}^{k}\right) \psi_{s}^{\prime}: \alpha_{i} \in \mathbb{F}_{n_{i}}^{+}, s \in\{1, \ldots, N\}\right\}$ are orthonormal bases for $\mathcal{M}$. Define the unitary operator $U: \mathcal{M} \rightarrow \mathcal{M}$ by setting

$$
U\left(\left(e_{\alpha_{1}}^{1} \otimes \cdots \otimes e_{\alpha_{k}}^{k}\right) \psi_{s}\right):=\left(e_{\alpha_{1}}^{1} \otimes \cdots \otimes e_{\alpha_{k}}^{k}\right) \psi_{s} .
$$

It is clear than $U\left(\left.\mathbf{S}_{i, j}\right|_{\mathcal{M}}\right)=\left(\left.\mathbf{S}_{i, j}\right|_{\mathcal{M}}\right) U$ for any $i \in\{1, \ldots, k\}$ and $j \in\left\{1, \ldots, n_{i}\right\}$. The proof is complete.

If $\mathcal{M}$ is a Beurling type invariant subspace of $\mathbf{S} \otimes I_{\mathcal{H}}$, then $\left.\left(\mathbf{S} \otimes I_{\mathcal{H}}\right)\right|_{\mathcal{M}}:=\left(\left.\left(\mathbf{S}_{1} \otimes I_{\mathcal{H}}\right)\right|_{\mathcal{M}}, \ldots,\left.\left(\mathbf{S}_{k} \otimes I_{\mathcal{H}}\right)\right|_{\mathcal{M}}\right)$ is in the polyball $\mathbf{B}_{\mathbf{n}}(\mathcal{M})$, where $\left.\left(\mathbf{S}_{i} \otimes I_{\mathcal{H}}\right)\right|_{\mathcal{M}}:=\left(\left.\left(\mathbf{S}_{i, 1} \otimes I_{\mathcal{H}}\right)\right|_{\mathcal{M}}, \ldots,\left.\left(\mathbf{S}_{i, n_{i}} \otimes I_{\mathcal{H}}\right)\right|_{\mathcal{M}}\right)$. We say that $\mathcal{M}$ has finite rank if $\left.\left(\mathbf{S} \otimes I_{\mathcal{H}}\right)\right|_{\mathcal{M}}$ has finite rank. The next result shows that the Euler characteristic completely classifies the finite rank Beurling type invariant subspaces of $\mathbf{S} \otimes I_{\mathcal{H}}$ which do not contain reducing subspaces. In particular, the Euler characteristic classifies the finite rank Beurling type invariant subspaces of $F^{2}\left(H_{n_{1}}\right) \otimes \cdots \otimes F^{2}\left(H_{n_{k}}\right)$.

Theorem 2.3. Let $\mathcal{M}$ and $\mathcal{N}$ be invariant subspaces of $\otimes_{i=1}^{k} F^{2}\left(H_{n_{i}}\right) \otimes \mathcal{H}$ which do not contain reducing subspaces of $\mathbf{S} \otimes I_{\mathcal{H}}$ and such that the defect operators $\Delta_{\mathcal{M}}$ and $\Delta_{\mathcal{N}}$ are positive and have finite ranks. Then $\mathcal{M}$ and $\mathcal{N}$ are unitarily equivalent if and only if

$$
\chi\left(\left.\left(\mathbf{S} \otimes I_{\mathcal{H}}\right)\right|_{\mathcal{M}}\right)=\chi\left(\left.\left(\mathbf{S} \otimes I_{\mathcal{H}}\right)\right|_{\mathcal{N}}\right) .
$$


Proof. Let $\mathcal{M}$ be a Beurling type invariant subspaces of $\otimes_{i=1}^{k} F^{2}\left(H_{n_{i}}\right) \otimes \mathcal{H}$. Then there is a Hilbert space $\mathcal{L}$ and an isometric multi-analytic operator $\Psi: \otimes_{i=1}^{k} F^{2}\left(H_{n_{i}}\right) \otimes \mathcal{L} \rightarrow \otimes_{i=1}^{k} F^{2}\left(H_{n_{i}}\right) \otimes \mathcal{H}$ such that $\mathcal{M}=\Psi\left[\otimes_{i=1}^{k} F^{2}\left(H_{n_{i}}\right) \otimes \mathcal{L}\right]$. Consequently, we have $P_{\mathcal{M}}=\Psi \Psi^{*}$ and

$$
\begin{aligned}
\boldsymbol{\Delta}_{\left.(\mathbf{S} \otimes I)\right|_{\mathcal{M}}}\left(I_{\mathcal{M}}\right) & =\left(i d-\Phi_{\left.\left(\mathbf{S}_{1} \otimes I\right)\right|_{\mathcal{M}}}\right) \circ \cdots \circ\left(i d-\Phi_{\left.\left(\mathbf{S}_{k} \otimes I\right)\right|_{\mathcal{M}}}\right)\left(P_{\mathcal{M}}\right) \\
& =\left.\Psi\left(i d-\Phi_{\mathbf{S}_{1} \otimes I}\right) \circ \cdots \circ\left(i d-\Phi_{\mathbf{S}_{k} \otimes I}\right)(I) \Psi^{*}\right|_{\mathcal{M}}=\left.\Psi\left(\mathbf{P}_{\mathbb{C}} \otimes I_{\mathcal{L}}\right) \Psi^{*}\right|_{\mathcal{M}}
\end{aligned}
$$

If $\left\{\ell_{\omega}\right\}_{\omega \in \Omega}$ is an orthonormal basis for $\mathcal{L}$, then $\left\{v_{\omega}:=\Psi\left(1 \otimes \ell_{\omega}\right): \omega \in \Omega\right\}$ is an orthonormal set and

$$
\left\{\Psi\left(e_{\beta_{1}}^{1} \otimes \cdots \otimes e_{\beta_{k}}^{k} \otimes \ell_{\omega}\right): \beta_{i} \in \mathbb{F}_{n_{i}}^{+}, i \in\{1, \ldots, k\}, \omega \in \Omega\right\}
$$

is an orthonormal basis for $\mathcal{M}$. It is easy to see that $\overline{\Psi\left(\mathbf{P}_{\mathbb{C}} \otimes I_{\mathcal{L}}\right) \Psi^{*}(\mathcal{M})}$ coincides with the closure of the range of the defect operator $\boldsymbol{\Delta}_{\left.(\mathbf{S} \otimes I)\right|_{\mathcal{M}}}\left(I_{\mathcal{M}}\right)$ and also to the closed linear span of $\left\{v_{\omega}:=\Psi\left(1 \otimes \ell_{\omega}\right): \omega \in \Omega\right\}$. This shows that

$$
\operatorname{rank}\left[\left.(\mathbf{S} \otimes I)\right|_{\mathcal{M}}\right]=\operatorname{card} \Omega=\operatorname{dim} \mathcal{L} .
$$

Assume that $\left.\left.\operatorname{rank}(\mathbf{S} \otimes I)\right|_{\mathcal{M}}\right)=p=\operatorname{dim} \mathcal{L}$. Taking into account that $\Psi$ is a multi-analytic operator and $P_{\mathcal{M}}=\Psi \Psi^{*}$, we deduce that

$$
\begin{aligned}
\left(i d-\Phi_{\left.\left(\mathbf{S}_{1} \otimes I\right)\right|_{\mathcal{M}}}^{q_{1}}\right) \circ \cdots \circ\left(i d-\Phi_{\left(\mathbf{S}_{k} \otimes I\right) \mid \mathcal{M}}^{q_{k}+1}\right)\left(I_{\mathcal{M}}\right) & =\left(i d-\Phi_{\mathbf{S}_{1} \otimes I}^{q_{1}+1}\right) \circ \cdots \circ\left(i d-\Phi_{\mathbf{S}_{k} \otimes I}^{q_{k}+1}\right)\left(P_{\mathcal{M}}\right) \\
& =\left.\Psi\left(i d-\Phi_{\mathbf{S}_{1} \otimes I_{\mathcal{L}}}^{q_{1}+1}\right) \circ \cdots \circ\left(i d-\Phi_{\mathbf{S}_{k} \otimes I_{\mathcal{L}}}^{q_{k}+1}\right)(I) \Psi^{*}\right|_{\mathcal{M}} .
\end{aligned}
$$

Hence, we have

$$
\begin{aligned}
\operatorname{rank}[(i d & \left.\left.-\Phi_{\left(\mathbf{S}_{1} \otimes I\right) \mid \mathcal{M}}^{q_{1}+1}\right) \circ \cdots \circ\left(i d-\Phi_{\left(\mathbf{S}_{k} \otimes I\right) \mid \mathcal{M}}^{q_{k}+1}\right)\left(I_{\mathcal{M}}\right)\right] \\
& =\operatorname{rank}\left[\left(i d-\Phi_{\mathbf{S}_{1} \otimes I_{\mathcal{L}}}^{q_{1}+1}\right) \circ \cdots \circ\left(i d-\Phi_{\mathbf{S}_{k} \otimes I_{\mathcal{L}}}^{q_{k}+1}\right)(I)\right] \\
& =\operatorname{rank}\left[\left(i d-\Phi_{\mathbf{S}_{1}}^{q_{1}+1}\right) \circ \cdots \circ\left(i d-\Phi_{\mathbf{S}_{k}}^{q_{k}+1}\right)(I)\right] \operatorname{dim} \mathcal{L} .
\end{aligned}
$$

Due to Theorem 1.2 and the fact that $\chi(\mathbf{S})=1$, we deduce that

$$
\chi\left(\left.(\mathbf{S} \otimes I)\right|_{\mathcal{M}}\right)=\operatorname{dim} \mathcal{L}=p=\operatorname{rank}\left[\left.(\mathbf{S} \otimes I)\right|_{\mathcal{M}}\right] .
$$

If $\mathcal{M}$ and $\mathcal{N}$ are finite rank Beurling type invariant subspaces of $\otimes_{i=1}^{k} F^{2}\left(H_{n_{i}}\right) \otimes \mathcal{H}$ and $\left.(\mathbf{S} \otimes I)\right|_{\mathcal{M}}$ is unitarily equivalent to $\left.(\mathbf{S} \otimes I)\right|_{\mathcal{N}}$, then $\chi\left(\left.(\mathbf{S} \otimes I)\right|_{\mathcal{M}}\right)=\chi\left(\left.(\mathbf{S} \otimes I)\right|_{\mathcal{N}}\right)$. To prove the converse, assume that the latter equality holds. Due to the first part of the proof, we must have $\operatorname{rank}\left(\left.(\mathbf{S} \otimes I)\right|_{\mathcal{M}}\right)=\operatorname{rank}\left(\left.(\mathbf{S} \otimes I)\right|_{\mathcal{N}}\right)$. This shows that the defect spaces associated with $\left.(\mathbf{S} \otimes I)\right|_{\mathcal{M}}$ and $\left.(\mathbf{S} \otimes I)\right|_{\mathcal{N}}$ have the same dimension. Now, the Wold decomposition from $\left[22\right.$ implies that $\left.(\mathbf{S} \otimes I)\right|_{\mathcal{M}}$ is unitarily equivalent to $\left.(\mathbf{S} \otimes I)\right|_{\mathcal{N}}$. The proof is complete.

Let $\mathcal{M}$ be an invariant subspace of the tensor product $F^{2}\left(H_{n_{1}}\right) \otimes \cdots \otimes F^{2}\left(H_{n_{k}}\right) \otimes \mathcal{E}$, where $\mathcal{E}$ is a finite dimensional Hilbert space. We introduce the Euler characteristic of of $\mathcal{M}^{\perp}$ by setting

$$
\chi\left(\mathcal{M}^{\perp}\right):=\lim _{q_{1} \rightarrow \infty} \cdots \lim _{q_{k} \rightarrow \infty} \frac{\operatorname{rank}\left[P_{\mathcal{M}^{\perp}}\left(P_{\leq\left(q_{1}, \ldots, q_{k}\right)} \otimes I_{\mathcal{E}}\right)\right]}{\operatorname{rank}\left[P_{\leq\left(q_{1}, \ldots, q_{k}\right)}\right]} .
$$

Theorem 2.4. Let $\mathcal{M}$ be an invariant subspace of the tensor product $F^{2}\left(H_{n_{1}}\right) \otimes \cdots \otimes F^{2}\left(H_{n_{k}}\right) \otimes \mathcal{E}$, where $\mathcal{E}$ is a finite dimensional Hilbert space. Then the Euler characteristic of $\mathcal{M}^{\perp}$ exists and satisfies the equation

$$
\chi\left(\mathcal{M}^{\perp}\right)=\chi(\mathbf{M})
$$

where $\mathbf{M}:=\left(M_{1}, \ldots, M_{k}\right)$ with $M_{i}:=\left(M_{i, 1}, \ldots, M_{i, n_{i}}\right)$ and $M_{i, j}:=\left.P_{\mathcal{M}^{\perp}}\left(\mathbf{S}_{i, j} \otimes I_{\mathcal{E}}\right)\right|_{\mathcal{M}^{\perp}}$.

Proof. Taking into account that $\mathcal{M}$ is an invariant subspace under each operator $\mathbf{S}_{i, j} \otimes I_{\mathcal{E}}$ for $i \in$ $\{1, \ldots, k\}, j \in\left\{1, \ldots, n_{i}\right\}$, we deduce that $M_{i, j}^{*} M_{r, s}^{*}=\left.\left(\mathbf{S}_{i, j}^{*} \otimes I_{\mathcal{E}}\right)\left(\mathbf{S}_{r, s}^{*} \otimes I_{\mathcal{E}}\right)\right|_{\mathcal{M}^{\perp}}$. Consequently, we have

$$
\boldsymbol{\Delta}_{\mathbf{M}}^{\mathbf{p}}\left(I_{\mathcal{M}^{\perp}}\right)=\left.P_{\mathcal{M}^{\perp}} \boldsymbol{\Delta}_{\mathbf{S} \otimes I}^{\mathbf{p}}(I)\right|_{\mathcal{M}^{\perp}} \geq 0
$$


for any $\mathbf{p}=\left(p_{1}, \ldots, p_{k}\right)$ with $p_{i} \in\{0,1\}$. Therefore, $\mathbf{M}$ is in the polyball $\mathbf{B}_{\mathbf{n}}\left(\mathcal{M}^{\perp}\right)$ and has finite rank. Since $\mathcal{M}^{\perp}$ is invariant under $\mathbf{S}_{i, j}^{*} \otimes I_{\mathcal{E}}$, we deduce that

$$
\begin{aligned}
\operatorname{rank}\left[\left(i d-\Phi_{M_{1}}^{q_{1}+1}\right) \circ \cdots \circ\left(i d-\Phi_{M_{k}}^{q_{k}+1}\right)\left(I_{\mathcal{M}^{\perp}}\right)\right] & =\operatorname{rank}\left[\left.P_{\mathcal{M}^{\perp}}\left(i d-\Phi_{\mathbf{S}_{1} \otimes I}^{q_{1}+1}\right) \circ \cdots \circ\left(i d-\Phi_{\mathbf{S}_{k} \otimes I}^{q_{k}+1}\right)(I)\right|_{\mathcal{M}^{\perp}}\right] \\
& =\operatorname{rank}\left[\left.P_{\mathcal{M}^{\perp}}\left(P_{\leq\left(q_{1}, \ldots, q_{k}\right)} \otimes I_{\mathcal{E}}\right)\right|_{\mathcal{M}^{\perp}}\right] \\
& =\operatorname{rank}\left[P_{\mathcal{M}^{\perp}}\left(P_{\leq\left(q_{1}, \ldots, q_{k}\right)} \otimes I_{\mathcal{E}}\right)\right]
\end{aligned}
$$

for any $q_{i} \in \mathbb{Z}_{+}$. Hence and using Theorem 1.2 , we conclude that $\chi(\mathbf{M})$ exists and

$$
\chi(\mathbf{M})=\lim _{q_{1} \rightarrow \infty} \cdots \lim _{q_{k} \rightarrow \infty} \frac{\operatorname{rank}\left[P_{\mathcal{M}^{\perp}}\left(P_{\leq\left(q_{1}, \ldots, q_{k}\right)} \otimes I_{\mathcal{E}}\right)\right]}{\operatorname{rank}\left[P_{\leq\left(q_{1}, \ldots, q_{k}\right)}\right]}=\chi\left(\mathcal{M}^{\perp}\right) .
$$

This completes the proof.

In what follows, we prove a multiplicative property for the Euler characteristic.

Theorem 2.5. Given a function $\kappa: \mathbb{N} \rightarrow \mathbb{N}$ and $\mathbf{n}^{(i)} \in \mathbb{N}^{\kappa(i)}$ for $i \in\{1, \ldots, p\}$, let $\mathbf{S}^{\left(\mathbf{n}^{(i)}\right)}$ and $\mathbf{S}^{\left(\mathbf{n}^{(1)}, \ldots, \mathbf{n}^{(p)}\right)}$ be the universal models of the polyballs $\mathbf{B}_{\mathbf{n}^{(i)}}$ and $\mathbf{B}_{\left(\mathbf{n}^{(1)}, \ldots, \mathbf{n}^{(p)}\right)}$, respectively. For each $i \in\{1, \ldots, p\}$, assume that

(i) $\mathcal{E}_{i}$ is a finite dimensional Hilbert space;

(ii) $\mathcal{M}_{i}$ is an invariant subspace under $\mathbf{S}^{\left(\mathbf{n}^{(i)}\right)} \otimes I_{\mathcal{E}_{i}}$.

Then the Euler characteristic satisfies the equation

$$
\chi\left(\mathcal{M}_{1}^{\perp} \otimes \cdots \otimes \mathcal{M}_{p}^{\perp}\right)=\prod_{i=1}^{p} \chi\left(\mathcal{M}_{i}^{\perp}\right)
$$

where, under the appropriate identification, $\mathcal{M}_{1}^{\perp} \otimes \cdots \otimes \mathcal{M}_{p}^{\perp}$ is viewed as a coinvariant subspace for

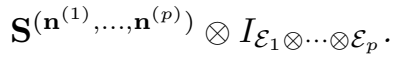

Proof. For each $i \in\{1, \ldots, p\}$ let $\mathbf{n}^{(i)}:=\left(n_{1}^{(i)}, \ldots, n_{\kappa(i)}^{(i)}\right) \in \mathbb{N}^{\kappa(i)}$. Given $j \in\{1, \ldots, \kappa(i)\}$, let $F^{2}\left(H_{n_{j}}^{(i)}\right)$ be the full Fock space with $n_{j}^{(i)}$ generators, and denote by $P_{q_{j}^{(i)}}^{\left(n_{j}^{(i)}\right)}$ the orthogonal projection of $F^{2}\left(H_{n_{j}^{(i)}}\right)$ onto the span of all homogeneous polynomials of $F^{2}\left(H_{n_{j}^{(i)}}\right)$ of degree equal to $q_{j}^{(i)} \in \mathbb{Z}_{+}$. Consider the orthogonal projections

$$
Q_{1}:=P_{\leq q_{1}^{(1)}}^{\left(n_{1}^{(1)}\right)} \otimes \cdots \otimes P_{\leq q_{\kappa(1)}^{(1)}}^{\left(n_{(1)}^{(1)}\right)}, \quad \cdots, \quad Q_{p}:=P_{\leq q_{1}^{(p)}}^{\left(n_{(p)}^{(p)}\right.} \otimes \cdots \otimes P_{\leq q_{\kappa(p)}^{(p)}}^{\left(n_{\kappa(p)}^{(p)}\right)}
$$

and let $U$ be the unitary operator which provides the canonical identification of the Hilbert tensor product $\otimes_{i=1}^{p}\left[F^{2}\left(H_{n_{1}}^{(i)}\right) \otimes \cdots \otimes F^{2}\left(H_{n_{\kappa(i)}}^{(i)}\right) \otimes \mathcal{E}_{i}\right]$ with $\left\{\otimes_{i=1}^{p}\left[F^{2}\left(H_{n_{1}}^{(i)}\right) \otimes \cdots \otimes F^{2}\left(H_{n_{\kappa(i)}}^{(i)}\right)\right]\right\} \otimes\left(\mathcal{E}_{1} \otimes \cdots \otimes \mathcal{E}_{p}\right)$. Note that

$$
U\left[\left(Q_{1} \otimes I_{\mathcal{E}_{1}}\right) \otimes \cdots \otimes\left(Q_{p} \otimes I_{\mathcal{E}_{p}}\right)\right]=\left[Q_{1} \otimes \cdots \otimes Q_{p} \otimes I_{\mathcal{E}_{1} \otimes \cdots \otimes \mathcal{E}_{p}}\right] U
$$

and $U\left(\mathcal{M}_{1}^{\perp} \otimes \cdots \otimes \mathcal{M}_{p}^{\perp}\right)$ is a coinvariant subspace under $\mathbf{S}^{\left(\mathbf{n}^{(1)}, \ldots, \mathbf{n}^{(p)}\right)} \otimes I_{\mathcal{E}_{1} \otimes \cdots \otimes \mathcal{E}_{p}}$. Due to Theorem 2.4 . we obtain

$$
\begin{aligned}
\chi\left(\otimes_{i=1}^{k} \mathcal{M}_{i}^{\perp}\right) & =\lim \frac{\operatorname{rank}\left[P_{U\left(\mathcal{M}_{1}^{\perp} \otimes \cdots \otimes \mathcal{M}_{p}^{\perp}\right)}\left(Q_{1} \otimes \cdots \otimes Q_{p} \otimes I_{\mathcal{E}_{1} \otimes \cdots \otimes \mathcal{E}_{p}}\right)\right]}{\operatorname{rank}\left[Q_{1} \otimes \cdots \otimes Q_{p}\right]} \\
& =\lim \frac{\operatorname{rank}\left[U^{*} P_{U\left(\mathcal{M}_{1}^{\perp} \otimes \cdots \otimes \mathcal{M}_{p}^{\perp}\right)} U U^{*}\left(Q_{1} \otimes \cdots \otimes Q_{p} \otimes I_{\mathcal{E}_{1} \otimes \cdots \otimes \mathcal{E}_{p}}\right) U\right]}{\operatorname{rank}\left[Q_{1} \otimes \cdots \otimes Q_{p}\right]} \\
& =\lim \frac{\operatorname{rank}\left\{P_{\mathcal{M}_{1}^{\perp} \otimes \cdots \otimes \mathcal{M}_{p}^{\perp}}\left[\left(Q_{1} \otimes I_{\mathcal{E}_{1}}\right) \otimes \cdots \otimes\left(Q_{p} \otimes I_{\mathcal{E}_{p}}\right)\right]\right\}}{\operatorname{rank}\left[Q_{1} \otimes \cdots \otimes Q_{p}\right]},
\end{aligned}
$$


where the limit is taken over $\left(q_{1}^{(1)}, \ldots, q_{\kappa(1)}^{(1)}, \ldots, q_{1}^{(p)}, \ldots, q_{\kappa(p)}^{(p)}\right) \in \mathbb{Z}_{+}^{\kappa(1)+\cdots+\kappa(p)}$. We remark that the latter limit is equal to the product

$$
\lim _{\left(q_{1}^{(1)}, \ldots, q_{\kappa(1)}^{(1)}\right) \in \mathbb{Z}_{+}^{\kappa(1)}} \frac{\operatorname{rank}\left[P_{\mathcal{M}_{1}^{\perp}}\left(Q_{1} \otimes I_{\mathcal{E}_{1}}\right)\right]}{\operatorname{rank}\left[Q_{1}\right]} \quad \cdots \quad \lim _{\left(q_{1}^{(p)}, \ldots, q_{\kappa(p)}^{(p)}\right) \in \mathbb{Z}_{+}^{\kappa(p)}} \frac{\operatorname{rank}\left[P_{\mathcal{M}_{p}^{\perp}}\left(Q_{p} \otimes I_{\mathcal{E}_{p}}\right)\right]}{\operatorname{rank}\left[Q_{p}\right]},
$$

which, due to Theorem 2.4 is equal to $\prod_{i=1}^{p} \chi\left(\mathcal{M}_{i}^{\perp}\right)$. Therefore, we have

$$
\chi\left(\otimes_{i=1}^{p} \mathcal{M}_{i}^{\perp}\right)=\prod_{i=1}^{p} \chi\left(\mathcal{M}_{i}^{\perp}\right) .
$$

The proof is complete.

As a particular case, we remark that if $\mathcal{M}^{\perp} \subset H^{2}\left(\mathbb{D}^{n}\right) \otimes \mathbb{C}^{r}$ and $\mathcal{N}^{\perp} \subset H^{2}\left(\mathbb{D}^{p}\right) \otimes \mathbb{C}^{q}$ are coinvariant subspaces, then so is $\mathcal{M}^{\perp} \otimes \mathcal{N}^{\perp} \subset H^{2}\left(\mathbb{D}^{n+p}\right) \otimes \mathbb{C}^{r q}$ and the Euler characteristic has the multiplicative property $\chi\left(\mathcal{M}^{\perp} \otimes \mathcal{N}^{\perp}\right)=\chi\left(\mathcal{M}^{\perp}\right) \chi\left(\mathcal{N}^{\perp}\right)$.

The next result shows that there are many non-isomorphic pure elements in the polyball with the same Euler characteristic.

Theorem 2.6. Let $\mathbf{n}=\left(n_{1}, \ldots, n_{k}\right) \in \mathbb{N}^{k}$ be such that $k \geq 2$ and $n_{i} \geq 2$ for all $i \in\{1, \ldots, k\}$. Then, for each $t \in(0,1)$, there exists an uncountable family $\left\{T^{(\omega)}(t)\right\}_{\omega \in \Omega}$ of pure elements in the regular polyball $\mathbf{B}_{\mathbf{n}}$ with the following properties:

(i) $T^{(\omega)}(t)$ in not unitarily equivalent to $T^{(\sigma)}(t)$ for any $\omega, \sigma \in \Omega, \omega \neq \sigma$.

(ii) $\operatorname{rank}\left[T^{(\omega)}(t)\right]=1$ and $\chi\left[T^{(\omega)}(t)\right]=t$ for all $\omega \in \Omega$.

Proof. If $t \in[0,1)$, there exists a subsequence of natural numbers $\left\{k_{p}\right\}_{p=1}^{N}, 1 \leq k_{1}<k_{2}<\cdots$, where $N \in \mathbb{N}$ or $N=\infty$, and $d_{p} \in\left\{1,2, \ldots, n_{i}-1\right\}$, such that $1-t=\sum_{p=1}^{N} \frac{d_{p}}{n_{i}^{k_{i}}}$. Define the following subsets of $\mathbb{F}_{n_{i}}^{+}$, the unital free semigroup on $n_{i}$ generators $g_{1}^{i}, \ldots, g_{n_{i}}^{i}$ and the identity $g_{0}^{i}$ :

$$
\begin{aligned}
& J_{1}^{i}:=\left\{\left(g_{1}^{i}\right)^{k_{1}}, \ldots,\left(g_{d_{1}}^{i}\right)^{k_{1}}\right\}, \\
& J_{p}^{1}:=\left\{\left(g_{1}^{i}\right)^{k_{p}-k_{p-1}}\left(g_{n_{i}}^{1}\right)^{k_{p-1}},\left(g_{2}^{i}\right)^{k_{p}-k_{p-1}}\left(g_{n_{i}}^{i}\right)^{k_{p-1}}, \ldots,\left(g_{d_{p}}^{i}\right)^{k_{p}-k_{p-1}}\left(g_{n_{i}}^{i}\right)^{k_{p-1}}\right\}, \quad p=2,3, \ldots, N .
\end{aligned}
$$

We remark that

$$
\mathcal{M}_{i}(t):=\bigoplus_{\beta \in \cup_{p=1}^{N} J_{p}^{i}} F^{2}\left(H_{n_{i}}\right) \otimes e_{\beta}^{i}
$$

is an invariant subspace of $F^{2}\left(H_{n_{i}}\right)$. Let $k_{p} \leq q_{i}<k_{p+1}$ and note that

$$
\begin{aligned}
\frac{\operatorname{rank}\left[P_{\mathcal{M}_{i}(t) \perp} P_{q_{i}}^{(i)}\right]}{\operatorname{rank}\left[P_{q_{i}}^{(i)}\right]} & =\frac{1}{\operatorname{rank}\left[P_{q_{i}}^{(i)}\right]} \sum_{\beta_{i} \in \mathbb{F}_{n_{i}}^{+}}\left\langle P_{\mathcal{M}_{i}(t) \perp} P_{q_{i}}^{(i)} e_{\beta_{i}}^{i}, e_{\beta_{i}}^{i}\right\rangle \\
& =1-\sum_{\beta_{i} \in \mathbb{F}_{n_{i}}^{+}} \frac{\left\langle P_{q_{i}}^{(i)} P_{\mathcal{M}_{i}(t)} P_{q_{i}}^{(i)} e_{\beta_{i}}^{i}, e_{\beta_{i}}^{i}\right\rangle}{n_{i}^{q_{i}}}=1-\frac{1}{n_{i}^{q_{i}}} \sum_{\beta_{i} \in \mathbb{F}_{n_{i}}^{+},\left|\beta_{i}\right|=q_{i}}\left\|P_{\mathcal{M}_{i}(t)} e_{\beta_{i}}^{i}\right\|^{2} \\
& =1-\frac{d_{1} n_{i}^{q_{i}-k_{1}}+\cdots+d_{p} n_{i}^{q_{i}-k_{p}}}{n_{i}^{q_{i}}}=1-\left(\frac{d_{1}}{n_{i}^{k_{1}}}+\cdots+\frac{d_{p}}{n_{i}^{k_{p}}}\right) .
\end{aligned}
$$

Consequently, we have

$$
\lim _{q_{i} \rightarrow \infty} \frac{\operatorname{rank}\left[P_{\mathcal{M}_{i}(t)^{\perp}} P_{q_{i}}^{(i)}\right]}{\operatorname{rank}\left[P_{q_{i}}^{(i)}\right]}=1-\sum_{p=1}^{N} \frac{d_{p}}{n_{1}^{k_{p}}}=t .
$$


Now, using Theorem 2.4 (when $k=1$ ) and the Stoltz-Cesaro limit theorem, we deduce that

$$
\begin{aligned}
\chi_{i}\left[\left.P_{\mathcal{M}_{i}(t)^{\perp}} \mathbf{S}_{i}\right|_{\mathcal{M}_{i}(t)^{\perp}}\right] & =\lim _{q_{i} \rightarrow \infty} \frac{\operatorname{rank}\left[P_{\mathcal{M}_{i}(t)^{\perp}} P_{\leq q_{i}}^{(i)}\right]}{\operatorname{rank}\left[P_{\leq q_{i}}^{(i)}\right]} \\
& =\lim _{q_{i} \rightarrow \infty} \frac{\sum_{s_{i}=0}^{q_{i}} \operatorname{rank}\left[P_{\mathcal{M}_{i}(t) \perp} P_{s_{i}}^{(i)}\right]}{\sum_{s_{i}=0}^{q_{i}} \operatorname{rank}\left[P_{s_{i}}^{(i)}\right]} \\
& =\lim _{q_{i} \rightarrow \infty} \frac{\operatorname{rank}\left[P_{\mathcal{M}_{i}(t)^{\perp}} P_{q_{i}}^{(i)}\right]}{\operatorname{rank}\left[P_{q_{i}}^{(i)}\right]}=1-\sum_{p=1}^{N} \frac{d_{p}}{n_{1}^{k_{p}}}=t
\end{aligned}
$$

where $\chi_{i}$ stands for the Euler characteristic on the polyball $\mathbf{B}_{n_{i}}$. Let $t \in(0,1)$ and $\omega \in(t, 1)$, and define the subspace

$$
\mathcal{M}^{(\omega)}(t):=\left(\mathcal{M}_{1}(\omega)^{\perp} \otimes \mathcal{M}_{2}\left(\frac{t}{\omega}\right)^{\perp} \otimes F^{2}\left(H_{n_{3}}\right) \otimes \cdots \otimes F^{2}\left(H_{n_{k}}\right)\right)^{\perp}
$$

Note that $\mathcal{M}^{(\omega)}(t)$ is an invariant subspace under $\mathbf{S}_{i, j}$ for any $i \in\{1, \ldots, k\}$ and $j \in\left\{1, \ldots, n_{i}\right\}$. Setting $T^{(\omega)}(t):=\left.P_{\mathcal{M}^{(\omega)}(t)^{\perp}} \mathbf{S}\right|_{\mathcal{M}^{(\omega)}(t)^{\perp}}$ and using Theorem 2.5, we deduce that

$$
\chi\left[T^{(\omega)}(t)\right]=\chi_{1}\left(\left.P_{\mathcal{M}_{1}(\omega) \perp} \mathbf{S}_{1}\right|_{\mathcal{M}_{1}(\omega) \perp}\right) \chi_{2}\left(\left.P_{\mathcal{M}_{2}\left(\frac{t}{\omega}\right)^{\perp}} \mathbf{S}_{2}\right|_{\mathcal{M}_{2}\left(\frac{t}{\omega}\right)^{\perp}}\right)=t .
$$

Let $\sigma \in(0, t)$. Since the Euler characteristic is a unitary invariant and

$$
\chi_{1}\left[\left.P_{\mathcal{M}_{1}(\omega) \perp} \mathbf{S}_{1}\right|_{\mathcal{M}_{1}(\omega) \perp}\right]=\omega \neq \sigma=\chi_{1}\left[\left.P_{\mathcal{M}_{1}(\sigma)^{\perp}} \mathbf{S}_{1}\right|_{\mathcal{M}_{1}(\sigma)^{\perp}}\right]
$$

we deduce that $\left.P_{\mathcal{M}_{1}(\omega) \perp} \mathbf{S}_{1}\right|_{\mathcal{M}_{1}(\omega) \perp}$ is not unitary equivalent to $\left.P_{\mathcal{M}_{1}(\sigma)^{\perp}} \mathbf{S}_{1}\right|_{\mathcal{M}_{1}(\sigma)^{\perp}}$. According to Theorem 2.10 from $\left[24\right.$, when $\mathcal{E}=\mathbb{C}$, we have $\mathcal{M}_{1}(\omega) \neq \mathcal{M}_{1}(\sigma)$, which implies $\mathcal{M}^{(\omega)}(t) \neq \mathcal{M}^{(\sigma)}(t)$. Using again the above-mentioned result from 24 , we conclude that $T^{(\omega)}(t)$ in not unitarily equivalent to $T^{(\sigma)}(t)$ for any $\omega, \sigma \in(0, t), \omega \neq \sigma$. The fact that $\operatorname{rank}\left[T^{(\omega)}(t)\right]=1$ is obvious. The proof is complete.

Corollary 2.7. Let $\mathbf{n}=\left(n_{1}, \ldots, n_{k}\right) \in \mathbb{N}^{k}$ be such that $n_{i} \geq 2$ and let $t \in[0,1]$. Then there exists a pure element $\mathbf{T}$ in the polyball $\mathbf{B}_{\mathbf{n}}$ such that $\operatorname{rank}(\mathbf{T})=1$ and

$$
\chi(\mathbf{T})=t .
$$

Proof. If $t=1$, the Euler characteristic $\chi(\mathbf{S})=1$. When $t \in[0,1)$, we consider the subspace $\mathcal{M}(t):=$ $\left(\mathcal{M}_{1}(t)^{\perp} \otimes F^{2}\left(H_{n_{2}}\right) \otimes \cdots \otimes F^{2}\left(H_{n_{k}}\right)\right)^{\perp}$, where $\mathcal{M}_{1}(t)$ is defined by relation (2.1). As in the proof of Theorem 2.6, one can show that $\chi\left(\left.P_{\mathcal{M}(t) \perp} \mathbf{S}\right|_{\mathcal{M}(t) \perp}\right)=t$. This completes the proof.

Using Corollary 2.7 and tensoring with the identity on $\mathbb{C}^{m}$, one can easily see that for any $t \in[0, m]$, there exists a pure element $\mathbf{T}$ in the polyball $\mathbf{B}_{\mathbf{n}}$, with $\operatorname{rank}(\mathbf{T})=m$ and $\chi(\mathbf{T})=t$. Consequently, the range of the Euler characteristic coincides with the interval $[0, \infty)$.

Theorem 2.8. Let $\mathbf{T} \in \mathbf{B}_{\mathbf{n}}(\mathcal{H})$ have finite rank and let $\mathcal{M}$ be an invariant subspace under $\mathbf{T}$ such that $\left.\mathbf{T}\right|_{\mathcal{M}} \in \mathbf{B}_{\mathbf{n}}(\mathcal{M})$ and $\operatorname{dim} \mathcal{M}^{\perp}<\infty$. Then $\left.\mathbf{T}\right|_{\mathcal{M}}$ has finite rank and

$$
\left|\chi(\mathbf{T})-\chi\left(\left.\mathbf{T}\right|_{\mathcal{M}}\right)\right| \leq \operatorname{dim} \mathcal{M}^{\perp} \prod_{i=1}^{k}\left(n_{i}-1\right) .
$$

Proof. First, note that $\operatorname{rank}\left(\left.\mathbf{T}\right|_{\mathcal{M}}\right)=\operatorname{rank} \boldsymbol{\Delta}_{\mathbf{T}}\left(P_{\mathcal{M}}\right)$. Taking into account the fact that $\boldsymbol{\Delta}_{\mathbf{T}}\left(P_{\mathcal{M}}\right)=$ $\boldsymbol{\Delta}_{\mathbf{T}}\left(I_{\mathcal{H}}\right)-\boldsymbol{\Delta}_{\mathbf{T}}\left(P_{\mathcal{M}^{\perp}}\right)$, we deduce that $\operatorname{rank}\left(\left.\mathbf{T}\right|_{\mathcal{M}}\right)<\infty$. On the other hand, since $\mathcal{M}$ is an invariant 
subspace under $\mathbf{T}$, we have

$$
\begin{aligned}
\operatorname{rank} & {\left[\left(i d-\Phi_{T_{1}||_{\mathcal{M}}}^{q_{1}+1}\right) \circ \cdots \circ\left(i d-\Phi_{\left.T_{k}\right|_{\mathcal{M}} ^{q_{k}+1}}\right)\left(I_{\mathcal{M}}\right)\right] } \\
& =\operatorname{rank}\left[\left(i d-\Phi_{T_{1}}^{q_{1}+1}\right) \circ \cdots \circ\left(i d-\Phi_{T_{k}}^{q_{k}+1}\right)\left(P_{\mathcal{M}}\right)\right] \\
& \leq \operatorname{rank}\left[\left(i d-\Phi_{T_{1}}^{q_{1}+1}\right) \circ \cdots \circ\left(i d-\Phi_{T_{k}}^{q_{k}+1}\right)\left(I_{\mathcal{H}}\right)\right]+\operatorname{rank}\left[\left(i d-\Phi_{T_{1}}^{q_{1}+1}\right) \circ \cdots \circ\left(i d-\Phi_{T_{k}}^{q_{k}+1}\right)\left(P_{\mathcal{M}^{\perp}}\right)\right] .
\end{aligned}
$$

Since

$$
\operatorname{rank}\left[\left(i d-\Phi_{T_{1}}^{q_{1}+1}\right) \circ \cdots \circ\left(i d-\Phi_{T_{k}}^{q_{k}+1}\right)\left(P_{\mathcal{M}^{\perp}}\right)\right] \leq\left(1+n_{1}^{q_{1}+1}\right) \cdots\left(1+n_{k}^{q_{k}+1}\right) \operatorname{rank}\left[P_{\mathcal{M}^{\perp}}\right],
$$

we deduce that

$$
\begin{gathered}
\left|\frac{\operatorname{rank}\left[\left(i d-\Phi_{T_{1}}^{q_{1}+1}\right) \circ \cdots \circ\left(i d-\Phi_{T_{k}}^{q_{k}+1}\right)\left(I_{\mathcal{H}}\right)\right]}{\prod_{i=1}^{k}\left(1+n_{i}+\cdots+n_{i}^{q_{i}}\right)}-\frac{\operatorname{rank}\left[\left(i d-\Phi_{T_{1} \mid \mathcal{M}}^{q_{1}+1}\right) \circ \cdots \circ\left(i d-\Phi_{\left.T_{k}\right|_{\mathcal{M}}}^{q_{k}+1}\right)\left(I_{\mathcal{M}}\right)\right]}{\prod_{i=1}^{k}\left(1+n_{i}+\cdots+n_{i}^{q_{i}}\right)}\right| \\
\leq \frac{\left(1+n_{1}^{q_{1}+1}\right) \cdots\left(1+n_{k}^{q_{k}+1}\right)}{\prod_{i=1}^{k}\left(1+n_{i}+\cdots+n_{i}^{q_{i}}\right)} \operatorname{trace}\left[P_{\mathcal{M}^{\perp}}\right] .
\end{gathered}
$$

Since $n_{i} \geq 2$, we can use Theorem 1.2 and obtain

$$
\left|\chi(\mathbf{T})-\chi\left(\left.\mathbf{T}\right|_{\mathcal{M}}\right)\right| \leq \operatorname{dim} \mathcal{M}^{\perp} \prod_{i=1}^{k}\left(n_{i}-1\right) .
$$

The proof is complete.

Theorem 2.9. Let $\mathbf{T} \in \mathbf{B}_{\mathbf{n}}(\mathcal{H})$ have finite rank and let $\mathcal{M}$ be a co-invariant subspace under $\mathbf{T}$ with $\operatorname{dim} \mathcal{M}^{\perp}<\infty$. Then $\left.P_{\mathcal{M}} \mathbf{T}\right|_{\mathcal{M}}$ has finite rank and

$$
\chi(\mathbf{T})=\chi\left(\left.P_{\mathcal{M}} \mathbf{T}\right|_{\mathcal{M}}\right)
$$

Proof. Denote $A:=\left(A_{1}, \ldots, A_{k}\right)$ and $A_{i}:=\left(A_{i, 1}, \ldots, A_{i, n_{i}}\right)$, where $A_{i, j}:=\left.P_{\mathcal{M}} T_{i, j}\right|_{\mathcal{M}}$ for $i \in\{1, \ldots, k\}$ and $j \in\left\{1, \ldots, n_{i}\right\}$. Using the fact that $\Phi_{A_{i}}^{q_{i}}\left(I_{\mathcal{M}}\right)=\left.P_{\mathcal{M}} \Phi_{T_{i}}^{q_{i}}\left(I_{\mathcal{H}}\right)\right|_{\mathcal{M}}$, we deduce that

$$
\begin{aligned}
& \operatorname{rank}\left[\left(i d-\Phi_{A_{1}}^{q_{1}+1}\right) \circ \cdots \circ\left(i d-\Phi_{A_{k}}^{q_{k}+1}\right)\left(I_{\mathcal{M}}\right)\right] \\
& \leq \operatorname{rank}\left[\left(i d-\Phi_{T_{1}}^{q_{1}+1}\right) \circ \cdots \circ\left(i d-\Phi_{T_{k}}^{q_{k}+1}\right)\left(I_{\mathcal{H}}\right)\right] \\
& \leq \operatorname{rank}\left[P_{\mathcal{M}}\left(i d-\Phi_{T_{1}}^{q_{1}+1}\right) \circ \cdots \circ\left(i d-\Phi_{T_{k}}^{q_{k}+1}\right)\left(I_{\mathcal{H}}\right)\right]+\operatorname{rank}\left[P_{\mathcal{M}^{\perp}}\left(i d-\Phi_{T_{1}}^{q_{1}+1}\right) \circ \cdots \circ\left(i d-\Phi_{T_{k}}^{q_{k}+1}\right)\left(I_{\mathcal{H}}\right)\right] \\
& \leq \operatorname{rank}\left[\left(i d-\Phi_{A_{1}}^{q_{1}+1}\right) \circ \cdots \circ\left(i d-\Phi_{A_{k}}^{q_{k}+1}\right)\left(I_{\mathcal{M}}\right)\right]+\operatorname{rank}\left[P_{\mathcal{M}^{\perp}}\left(i d-\Phi_{T_{1}}^{q_{1}+1}\right) \circ \cdots \circ\left(i d-\Phi_{T_{k}}^{q_{k}+1}\right)\left(I_{\mathcal{H}}\right)\right] .
\end{aligned}
$$

Note also that

$$
\operatorname{rank}\left[P_{\mathcal{M} \perp}\left(i d-\Phi_{T_{1}}^{q_{1}+1}\right) \circ \cdots \circ\left(i d-\Phi_{T_{k}}^{q_{k}+1}\right)\left(I_{\mathcal{H}}\right)\right] \leq \operatorname{dim} \mathcal{M}^{\perp} .
$$

Hence, we deduce that

$$
\begin{gathered}
\left|\frac{\operatorname{rank}\left[\left(i d-\Phi_{T_{1}}^{q_{1}+1}\right) \circ \cdots \circ\left(i d-\Phi_{T_{k}}^{q_{k}+1}\right)\left(I_{\mathcal{H}}\right)\right]}{\prod_{i=1}^{k}\left(1+n_{i}+\cdots+n_{i}^{q_{i}}\right)}-\frac{\operatorname{rank}\left[\left(i d-\Phi_{A_{1}}^{q_{1}+1}\right) \circ \cdots \circ\left(i d-\Phi_{A_{k}}^{q_{k}+1}\right)\left(I_{\mathcal{M}}\right)\right]}{\prod_{i=1}^{k}\left(1+n_{i}+\cdots+n_{i}^{q_{i}}\right)}\right| \\
\leq \frac{1}{\prod_{i=1}^{k}\left(1+n_{i}+\cdots+n_{i}^{q_{i}}\right)} \operatorname{dim} \mathcal{M}^{\perp} .
\end{gathered}
$$

Now, using Theorem 1.2, we conclude that $\chi(\mathbf{T})=\chi(\mathbf{A})$, which completes the proof.

According to [18, there are invariant subspaces $\mathcal{M}_{1} \subset F^{2}\left(H_{n_{1}}\right)$ which do not contain nonzero polynomials in $F^{2}\left(H_{n_{1}}\right)$. This implies that the set $\left\{P_{\mathcal{M}_{1}^{\perp}} e_{\alpha_{1}}^{1}\right\}_{\alpha_{1} \in \mathbb{F}_{n_{1}}^{+},\left|\alpha_{1}\right| \leq m}$ is linearly independent for any $m \in \mathbb{N}$. Consequently, the set $\left\{\left(P_{\mathcal{M}_{1}^{\perp}} \otimes I \otimes \cdots \otimes I\right)\left(e_{\alpha_{1}}^{1} \otimes \cdots \otimes e_{\alpha_{k}}^{k}\right)\right\}_{\alpha_{i} \in \mathbb{F}_{n_{i}}^{+},\left|\alpha_{i}\right| \leq m}$ is linearly independent for each $m \in \mathbb{N}$. This shows that the subspace $\mathcal{M}_{1} \otimes F^{2}\left(H_{n_{2}}\right) \otimes \cdots \otimes F^{2}\left(H_{n_{k}}\right)$ does not contain nonzero 
polynomials. On the other hand, according to [15] (see also [16]), any invariant subspace $\mathcal{M}_{1} \subset F^{2}\left(H_{n_{1}}\right.$ ) is of Beurling type. Therefore, $\mathcal{M}_{1} \otimes F^{2}\left(H_{n_{2}}\right) \otimes \cdots \otimes F^{2}\left(H_{n_{k}}\right)$ is also of Beurling type.

Lemma 2.10. Let $\mathcal{M} \subset F^{2}\left(H_{n_{1}}\right) \otimes \cdots \otimes F^{2}\left(H_{n_{k}}\right)$ be an nonzero invariant subspace satisfying either one of the following conditions:

(i) $\mathcal{M}$ is a Beurling type invariant subspace which does not contain any polynomial;

(ii) $\mathcal{M}=\left(\mathcal{M}_{1}^{\perp} \otimes \cdots \mathcal{M}_{k}^{\perp}\right)^{\perp}$, where $\{0\} \neq \mathcal{M}_{i} \subset F^{2}\left(H_{n_{i}}\right)$ are invariant subspaces which do not contain nonzero polynomials.

Then

$$
\operatorname{curv}\left(\left.P_{\mathcal{M}^{\perp}} \mathbf{S}\right|_{\mathcal{M}^{\perp}}\right)<1 \quad \text { and } \quad \chi\left(\left.P_{\mathcal{M}^{\perp}} \mathbf{S}\right|_{\mathcal{M}^{\perp}}\right)=1
$$

Proof. Assume that $\mathcal{M} \subset F^{2}\left(H_{n_{1}}\right) \otimes \cdots \otimes F^{2}\left(H_{n_{k}}\right)$ is an nonzero invariant subspace which does not contain any polynomial. Then the set $\left\{P_{\mathcal{M}^{\perp}}\left(e_{\alpha_{1}}^{1} \otimes \cdots \otimes e_{\alpha_{k}}^{k}\right)\right\}_{\alpha_{i} \in \mathbb{F}_{n_{i}}^{+},\left|\alpha_{i}\right| \leq m}$ is linearly independent for each $m \in \mathbb{N}$ and, consequently, using Theorem 2.4 we deduce that

$$
\chi\left(\left.P_{\mathcal{M}^{\perp}} \mathbf{S}\right|_{\mathcal{M}^{\perp}}\right)=1 .
$$

If, in addition, $\mathcal{M}$ is a Beurling type invariant subspace, then due to [24] (see Proposition 3.14), we have $\operatorname{curv}\left(\left.P_{\mathcal{M}^{\perp}} \mathbf{S}\right|_{\mathcal{M}^{\perp}}\right)<1$.

To prove part (ii), assume that $\mathcal{M}=\left(\mathcal{M}_{1}^{\perp} \otimes \cdots \mathcal{M}_{k}^{\perp}\right)^{\perp}$, where $\{0\} \neq \mathcal{M}_{i} \subset F^{2}\left(H_{n_{i}}\right)$ are invariant subspaces which do not contain nonzero polynomials. Due to the first part of the proof, when $k=1$, we have $\chi\left(\left.P_{\mathcal{M}_{i}^{\perp}} \mathbf{S}_{i}\right|_{\mathcal{M}_{i}^{\perp}}\right)=1$ and $\operatorname{curv}\left(\left.P_{\mathcal{M}_{i}^{\perp}} \mathbf{S}_{i}\right|_{\mathcal{M}_{i}^{\perp}}\right)<1$. Due to Theorem 2.4 and Theorem 2.5. we have

$$
\chi\left(\left.P_{\mathcal{M}^{\perp}} \mathbf{S}\right|_{\mathcal{M}^{\perp}}\right)=\chi\left(\mathcal{M}_{1}^{\perp} \otimes \cdots \mathcal{M}_{k}^{\perp}\right)=\prod_{i=1}^{k} \chi\left(\left.P_{\mathcal{M}_{i}^{\perp}} \mathbf{S}_{\mathbf{i}}\right|_{\mathcal{M}_{i}^{\perp}}\right)=1 .
$$

On the other hand, using [24] (see Corollary 2.5), we obtain

$$
\operatorname{curv}\left(\left.P_{\mathcal{M}^{\perp}} \mathbf{S}\right|_{\mathcal{M}^{\perp}}\right)=\operatorname{curv}\left(\left.P_{\mathcal{M}_{1}^{\perp} \otimes \cdots \mathcal{M}_{k}^{\perp}} \mathbf{S}\right|_{\mathcal{M}_{1}^{\perp} \otimes \cdots \mathcal{M}_{k}^{\perp}}\right)=\prod_{i=1}^{k} \operatorname{curv}\left(P_{\mathcal{M}_{i}^{\perp}} \mathbf{S}_{\mathcal{M}_{i}^{\perp}}\right)<1
$$

This completes the proof.

The next result shows that the curvature and the Euler characteristic can be far from each each other.

Proposition 2.11. For every $\epsilon \in(0,1)$ and $m \in \mathbb{N}$, there exists $k \in \mathbb{N}$ and $\mathbf{T} \in \mathbf{B}_{\mathbf{n}}(\mathcal{H})$ with $\mathbf{n}=$ $\left(n_{1}, \ldots, n_{k}\right)$ and $n_{i} \geq 2$ such that

$$
0<\operatorname{curv}(\mathbf{T})<\epsilon \quad \text { and } \quad \chi(\mathbf{T})=\operatorname{rank}(\mathbf{T})=m .
$$

Proof. Due to Lemma 2.10, there is an invariant subspace $\mathcal{M}_{1} \subseteq F^{2}\left(H_{n_{1}}\right)$ such that $\chi\left(\left.P_{\mathcal{M}_{1}^{\perp}} \mathbf{S}_{1}\right|_{\mathcal{M}_{1}^{\perp}}\right)=1$ and $t:=\operatorname{curv}\left(\left.P_{\mathcal{M}_{1}^{\perp}} \mathbf{S}_{1}\right|_{\mathcal{M}_{1}^{\perp}}\right)<1$. Given $\epsilon>0$, take $k \in \mathbb{N}$ such that $t^{k}<\epsilon$. Consider $n_{1}=\cdots=n_{k}$ and let $\mathcal{M}:=\left(\mathcal{M}_{1}^{\perp} \otimes \cdots \mathcal{M}_{1}^{\perp}\right)^{\perp}$. As in the proof of Lemma 2.10, we have

$$
\operatorname{curv}\left(\left.P_{\mathcal{M}^{\perp}} \mathbf{S}\right|_{\mathcal{M}^{\perp}}\right)=t^{k}<\epsilon \quad \text { and } \chi\left(\left.P_{\mathcal{M}^{\perp}} \mathbf{S}\right|_{\mathcal{M}^{\perp}}\right)=1 .
$$

The proof is complete. 


\section{A Gauss-Bonnet-Chern type theorem on noncommutative polyballs}

In this section, we provide a characterization of the graded invariant subspaces for the tensor product $F^{2}\left(H_{n_{1}}\right) \otimes \cdots \otimes F^{2}\left(H_{n_{k}}\right)$, and obtain a version of the Gauss-Bonnet-Chern theorem on noncommutative polyballs.

A Hilbert space $\mathcal{H}$ is called $\mathbb{Z}^{k}$-graded if there is a strongly continuous unitary representation $U: \mathbb{T}^{k} \rightarrow$ $B(\mathcal{H})$ of the $k$-torus $\mathbb{T}^{k}=\mathbb{T} \times \cdots \times \mathbb{T}$, where $\mathbb{T}:=\{z \in \mathbb{C}:|z|=1\}$. $U$ is called the gauge group of $\mathcal{H}$.

Definition 3.1. A tuple $\mathbf{T}=\left(T_{1}, \ldots, T_{k}\right) \in B(\mathcal{H})^{n_{1}} \times \cdots \times B(\mathcal{H})^{n_{k}}$ with $T_{i}=\left(T_{i, 1}, \ldots, T_{i, n_{i}}\right)$, is called $\mathbb{Z}^{k}$-graded if there is a distinguished gauge group $U$ on $\mathcal{H}$ such that

$$
U(\lambda) T_{i, j} U(\lambda)^{*}=\lambda_{i} T_{i, j}, \quad \lambda=\left(\lambda_{1}, \ldots, \lambda_{k}\right) \in \mathbb{T}^{k},
$$

for any $i \in\{1, \ldots, k\}$ and $j \in\left\{1, \ldots, n_{i}\right\}$.

A closed subspace $\mathcal{M} \subseteq \mathcal{H}$ is called graded with respect to $U$ if $U(\lambda) \mathcal{M} \subseteq \mathcal{M}$ for all $\lambda \in \mathbb{T}^{k}$. In this case, the subrepresentation $\left.U\right|_{\mathcal{M}}$ is the gauge group on $\mathcal{M}$. Similarly, the orthocomplement $\mathcal{M}^{\perp}:=\mathcal{H} \ominus \mathcal{M}$ is called graded if $U(\lambda) \mathcal{M}^{\perp} \subseteq \mathcal{M}^{\perp}$ for all $\lambda \in \mathbb{T}^{k}$. Note that $\mathcal{M}$ is graded if and only if $\mathcal{M}^{\perp}$ is graded.

Assume that $\mathbf{T}$ is graded with respect to the gauge group $U$ on $\mathcal{H}$ and let $\mathcal{M} \subseteq \mathcal{H}$ be an invariant subspace under $\mathbf{T}$. Note that if $\mathcal{M}$ is graded with respect to $U$, then $\left.\mathbf{T}\right|_{\mathcal{M}}$ is graded with respect to the subrepresentation $\left.U\right|_{\mathcal{M}}$, i.e.

$$
\left.U(\lambda)\right|_{\mathcal{M}}\left(\left.T_{i, j}\right|_{\mathcal{M}}\right)=\left.\lambda_{i}\left(\left.T_{i, j}\right|_{\mathcal{M}}\right) U(\lambda)\right|_{\mathcal{M}}, \quad \lambda=\left(\lambda_{1}, \ldots, \lambda_{k}\right) \in \mathbb{T}^{k}
$$

for any $i \in\{1, \ldots, k\}$ and $j \in\left\{1, \ldots, n_{i}\right\}$. Similarly, $\mathcal{M}^{\perp}$ is graded with respect to $U$ and $\left.P_{\mathcal{M}^{\perp}} \mathbf{T}\right|_{\mathcal{M}^{\perp}}$ is graded with respect to the subrepresentation $\left.U\right|_{\mathcal{M}^{\perp}}$.

We remark that the universal model $\mathbf{S}=\left(\mathbf{S}_{1}, \ldots, \mathbf{S}_{k}\right), \mathbf{S}_{i}=\left(\mathbf{S}_{i, 1}, \ldots, \mathbf{S}_{i, j}\right)$, with $\mathbf{S}_{i, j}$ acting on the tensor product $F^{2}\left(H_{n_{1}}\right) \otimes \cdots \otimes F^{2}\left(H_{n_{k}}\right)$, is $\mathbb{Z}^{k}$-graded with respect to the canonical gauge group $U: \mathbb{T}^{k} \rightarrow B\left(F^{2}\left(H_{n_{1}}\right) \otimes \cdots \otimes F^{2}\left(H_{n_{k}}\right)\right)$ defined by

$$
U(\lambda)\left(\sum_{\substack{\alpha_{i} \in \mathbb{E}_{n_{i}}^{+} \\ i \in\{1, \ldots, k\}}} a_{\alpha_{1}, \ldots, \alpha_{k}} e_{\alpha_{1}}^{1} \otimes \cdots \otimes e_{\alpha_{k}}^{k}\right)=\sum_{\substack{\alpha_{i} \in \mathbb{F}_{n_{i}}^{+} \\ i \in\{1, \ldots, k\}}} \lambda_{1}^{\left|\alpha_{1}\right|} \cdots \lambda_{k}^{|\alpha|} a_{\alpha_{1}, \ldots, \alpha_{k}} e_{\alpha_{1}}^{1} \otimes \cdots \otimes e_{\alpha_{k}}^{k} .
$$

Given $\left(s_{1}, \ldots, s_{k}\right) \in \mathbb{Z}_{+}^{k}$, we say that a polynomial in $F^{2}\left(H_{n_{1}}\right) \otimes \cdots \otimes F^{2}\left(H_{n_{k}}\right)$ is multi-homogeneous of order $\left(s_{1}, \ldots, s_{k}\right)$ if it has the form

$$
q=\sum_{\substack{\alpha_{i} \in \mathbb{P}_{n_{i}}^{+}, \alpha_{i} \mid=s_{i} \\ i \in\{1, \ldots, k\}}} a_{\alpha_{1}, \ldots, \alpha_{k}} e_{\alpha_{1}}^{1} \otimes \cdots \otimes e_{\alpha_{k}}^{k} .
$$

This is equivalent to the fact that $U(\lambda) q=\lambda_{1}^{s_{1}} \cdots \lambda_{k}^{s_{k}} q$ for any $\lambda=\left(\lambda_{1}, \ldots, \lambda_{k}\right) \in \mathbb{T}^{k}$.

Let $\mathcal{M} \subset F^{2}\left(H_{n_{1}}\right) \otimes \cdots \otimes F^{2}\left(H_{n_{k}}\right)$ be an invariant subspace for the universal model $\mathbf{S}$. We recall (see Corollary 2.2 from [24]) that the defect map $\boldsymbol{\Delta}_{\mathbf{S}}$ is one-to-one and any projection $P_{\mathcal{M}}$ has the following Taylor type representation around its defect

$$
P_{\mathcal{M}}=\sum_{s_{1}=0}^{\infty} \Phi_{\mathbf{S}_{1}}^{s_{1}}\left(\sum_{s_{2}=0}^{\infty} \Phi_{\mathbf{S}_{2}}^{s_{2}}\left(\cdots \sum_{s_{k}=0}^{\infty} \Phi_{\mathbf{S}_{k}}^{s_{k}}\left(\boldsymbol{\Delta}_{\mathbf{S}}\left(P_{\mathcal{M}}\right)\right) \cdots\right)\right)
$$

where the iterated series converge in the weak operator topology. If, in addition, $\boldsymbol{\Delta}_{\mathbf{S}}\left(P_{\mathcal{M}}\right) \geq 0$, then

$$
P_{\mathcal{M}}=\sum_{\left(s_{1}, \ldots, s_{k}\right) \in \mathbb{Z}_{+}^{k}} \Phi_{\mathbf{S}_{1}}^{s_{1}} \circ \cdots \circ \Phi_{\mathbf{S}_{k}}^{s_{k}}\left(\boldsymbol{\Delta}_{\mathbf{S}}\left(P_{\mathcal{M}}\right)\right) .
$$

We recall that defect operator of $\mathcal{M}$ is given by $\Delta_{\mathcal{M}}:=\Delta_{\mathbf{S}}\left(P_{\mathcal{M}}\right)$. Note that $\Delta_{\mathcal{M}}(\mathcal{M}) \subseteq \mathcal{M}$ and $\Delta_{\mathcal{M}}\left(\mathcal{M}^{\perp}\right)=\{0\}$. Moreover, we have $\Delta_{\left.\mathbf{S}\right|_{\mathcal{M}}}\left(I_{\mathcal{M}}\right)=\left.\Delta_{\mathcal{M}}\right|_{\mathcal{M}}$. Now, it is clear that $\Delta_{\mathcal{M}}$ is positive and has finite rank if and only if $\left.\boldsymbol{\Delta}_{\mathbf{S}}\right|_{\mathcal{M}}\left(I_{\mathcal{M}}\right)$ has the same properties. The remarks above show that the invariant subspace $\mathcal{M}$ is uniquely determined by its defect operator $\Delta_{\mathcal{M}}$. 
Note that any set $\left\{\psi_{s}\right\}_{s \in \Lambda}, \Lambda \subseteq \mathbb{N}$, of multi-homogeneous polynomials (perhaps of different orders) generates a graded closed invariant subspace of $F^{2}\left(H_{n_{1}}\right) \otimes \cdots \otimes F^{2}\left(H_{n_{k}}\right)$ by setting

$$
\mathcal{M}:=\overline{\operatorname{span}}\left\{\mathbf{S}_{1, \alpha_{1}} \cdots \mathbf{S}_{k, \alpha_{k}} \psi_{s}: \alpha \in \mathbb{F}_{n_{i}}^{+}, i \in\{1, \ldots, k\}, s \in \Lambda\right\} .
$$

The next theorem provides a characterization of those graded invariant subspaces $\mathcal{M} \subseteq F^{2}\left(H_{n_{1}}\right) \otimes \cdots \otimes$ $F^{2}\left(H_{n_{k}}\right)$ with positive defect operator $\Delta_{\mathcal{M}}$.

If $f:=\sum_{\beta_{i} \in \mathbb{F}_{n_{i}}^{+}} a_{\beta_{1}, \ldots, \beta_{k}} e_{\beta_{1}}^{1} \otimes \cdots \otimes e_{\beta_{k}}^{k}$ is a polynomial in $F^{2}\left(H_{n_{1}}\right) \otimes \cdots \otimes F^{2}\left(H_{n_{k}}\right)$, and $X_{i, j}$ are bounded operators on a Hilbert space, where $i \in\{1, \ldots, k\}$ and $j \in\left\{1, \ldots, n_{i}\right\}$, we define the polynomial calculus $f\left(X_{i, j}\right):=\sum_{\beta_{i} \in \mathbb{F}_{n_{i}}^{+}} a_{\beta_{1}, \ldots, \beta_{k}} X_{1, \beta_{1}} \cdots X_{k, \beta_{k}}$. We use the notation $\widetilde{f}\left(\mathbf{R}_{i, j}\right):=\sum_{\beta_{i} \in \mathbb{F}_{n_{i}}^{+}} a_{\beta_{1}, \ldots, \beta_{k}} \mathbf{R}_{1, \widetilde{\beta}_{1}} \cdots \mathbf{R}_{k, \widetilde{\beta}_{k}}$, where $\widetilde{\beta}_{i}=g_{j_{p}}^{i} \cdots g_{j_{1}}^{i}$ denotes the reverse of $\beta_{i}=g_{j_{1}}^{i} \cdots g_{j_{p}}^{i} \in \mathbb{F}_{n_{i}}^{+}$.

Theorem 3.2. Let $\mathcal{M} \subseteq F^{2}\left(H_{n_{1}}\right) \otimes \cdots \otimes F^{2}\left(H_{n_{k}}\right)$ be an invariant subspace. Then $\mathcal{M}$ is graded and has positive defect operator $\Delta_{\mathcal{M}}$ if and only if there is a sequence of multi-homogeneous polynomials $\left\{\psi_{s}\right\}_{s=1}^{N}$, where $N \in \mathbb{N}$ or $N=\infty$, with the following properties:

(i) each $\psi_{s}$ is in the range of $\Delta_{\mathcal{M}}$;

(ii) $\left\{\widetilde{\psi}_{s}\left(\mathbf{R}_{i, j}\right)\right\}_{s=1}^{N}$ are isometries with orthogonal ranges, where $\left\{\mathbf{R}_{i, j}\right\}$ is the right universal model;

(iii) The orthogonal projection $P_{\mathcal{M}}$ satisfies the relation

$$
P_{\mathcal{M}}=\sum_{s=1}^{N} \widetilde{\psi}_{s}\left(R_{i, j}\right) \widetilde{\psi}_{s}\left(R_{i, j}\right)^{*}
$$

where the convergence is in the strong operator topology.

Moreover, in this case, we have $N=\operatorname{rank}\left(\Delta_{\mathcal{M}}\right)$ and

$$
\Delta_{\mathcal{M}} \xi=\sum_{t=1}^{N}\left\langle\xi, \psi_{s}\right\rangle \psi_{s}, \quad \xi \in \otimes_{i=1}^{k} F^{2}\left(H_{n_{i}}\right)
$$

Proof. Assume that $\mathcal{M} \subseteq F^{2}\left(H_{n_{1}}\right) \otimes \cdots \otimes F^{2}\left(H_{n_{k}}\right)$ is a graded invariant subspace and has positive defect operator $\Delta_{\mathcal{M}}$. Let $U: \mathbb{T}^{k} \rightarrow B\left(F^{2}\left(H_{n_{1}}\right) \otimes \cdots \otimes F^{2}\left(H_{n_{k}}\right)\right)$ be the canonical gauge group of $F^{2}\left(H_{n_{1}}\right) \otimes \cdots \otimes F^{2}\left(H_{n_{k}}\right)$. Then $U\left(\lambda_{1}, \ldots, \lambda_{k}\right) \mathcal{M}=\mathcal{M}$ for any $\left(\lambda_{1}, \ldots, \lambda_{k}\right) \in \mathbb{T}^{k}$ and, consequently $U\left(\lambda_{1}, \ldots, \lambda_{k}\right)$ commutes with $P_{\mathcal{M}}$. Taking into account that

$$
U\left(\lambda_{1}, \ldots, \lambda_{k}\right) \mathbf{S}_{i, j} U\left(\lambda_{1}, \ldots, \lambda_{k}\right)^{*}=\lambda_{i} \mathbf{S}_{i, j}, \quad \lambda=\left(\lambda_{1}, \ldots, \lambda_{k}\right) \in \mathbb{T}^{k},
$$

for any $i \in\{1, \ldots, k\}$ and $j \in\left\{1, \ldots, n_{i}\right\}$, one can easily see that

$$
U\left(\lambda_{1}, \ldots, \lambda_{k}\right) \Delta_{\mathcal{M}}=\Delta_{\mathcal{M}} U\left(\lambda_{1}, \ldots, \lambda_{k}\right), \quad \lambda=\left(\lambda_{1}, \ldots, \lambda_{k}\right) \in \mathbb{T}^{k} .
$$

For each $\left(s_{1}, \ldots, s_{k}\right) \in \mathbb{Z}_{+}^{k}$, let $Q_{\left(s_{1}, \ldots, s_{k}\right)}$ be the orthogonal projection of $F^{2}\left(H_{n_{1}}\right) \otimes \cdots \otimes F^{2}\left(H_{n_{k}}\right)$ onto the subspace of multi-homogeneous polynomials of order $\left(s_{1}, \ldots, s_{k}\right)$. Since

$$
U\left(\lambda_{1}, \ldots, \lambda_{k}\right)=\sum_{p_{1}=0}^{\infty} \cdots \sum_{p_{k}=0}^{\infty} \lambda_{1}^{\alpha_{1}} \cdots \lambda_{k}^{\alpha} Q_{\left(s_{1}, \ldots, s_{k}\right)}, \quad \lambda=\left(\lambda_{1}, \ldots, \lambda_{k}\right) \in \mathbb{T}^{k},
$$

and due to the spectral theorem, we deduce that

$$
\Delta_{\mathcal{M}} Q_{\left(s_{1}, \ldots, s_{k}\right)}=Q_{\left(s_{1}, \ldots, s_{k}\right)} \Delta_{\mathcal{M}}, \quad\left(s_{1}, \ldots, s_{k}\right) \in \mathbb{Z}^{k} .
$$

If $\left(p_{1}, \ldots, p_{k}\right)$ is in the set

$$
\Omega:=\left\{\left(s_{1}, \ldots, s_{k}\right) \in \mathbb{Z}^{k}: \Delta_{\mathcal{M}} Q_{\left(s_{1}, \ldots, s_{k}\right)} \neq 0\right\},
$$

then $\Delta_{\mathcal{M}} Q_{\left(p_{1}, \ldots, p_{k}\right)}$ is a nonzero positive finite rank operator supported in the space of multi-homogeneous polynomials $Q_{\left(p_{1}, \ldots, p_{k}\right)}\left(\otimes_{i=1}^{k} F^{2}\left(H_{n_{i}}\right)\right)$. Due to the spectral theorem, it can be expressed as a finite sum $\Delta_{\mathcal{M}} Q_{\left(p_{1}, \ldots, p_{k}\right)}=\sum_{t=1}^{m} \Lambda_{t}$ of rank-one positive operators $\Lambda_{t}$ defined by

$$
\Lambda_{t} \xi:=\left\langle\xi, \psi_{t}\right\rangle \psi_{t}, \quad \xi \in \otimes_{i=1}^{k} F^{2}\left(H_{n_{i}}\right)
$$


where $\left\{\psi_{t}\right\}_{t=1}^{m}$ are orthonormal multi-homogeneous polynomials in $\Delta_{\mathcal{M}} Q_{\left(p_{1}, \ldots, p_{k}\right)}\left(\otimes_{i=1}^{k} F^{2}\left(H_{n_{i}}\right)\right)$. Note that

$$
\widetilde{\psi}_{t}\left(\mathbf{R}_{i, j}\right) P_{\mathbb{C}} \widetilde{\psi}_{t}\left(\mathbf{R}_{i, j}\right)^{*} \xi=\widetilde{\psi}_{t}\left(\mathbf{R}_{i, j}\right)\left(\left\langle\xi, \psi_{t}\right\rangle\right)=\left\langle\xi, \psi_{t}\right\rangle \psi_{t}=\Lambda_{t} \xi
$$

Consequently, using the fact that $\widetilde{\psi}_{t}\left(\mathbf{R}_{i, j}\right)$ is a multi-analytic operator with respect to the universal model $\mathbf{S}$, we have

$$
\sum_{s_{1}=0}^{q_{1}} \cdots \sum_{s_{k}=0}^{q_{k}} \Phi_{\mathbf{S}_{1}}^{s_{1}} \circ \cdots \circ \Phi_{\mathbf{S}_{k}}^{s_{k}}\left(\Lambda_{t}\right)=\widetilde{\psi}_{t}\left(\mathbf{R}_{i, j}\right)\left(\sum_{s_{1}=0}^{q_{1}} \cdots \sum_{s_{k}=0}^{q_{k}} \Phi_{\mathbf{S}_{1}}^{s_{1}} \circ \cdots \circ \Phi_{\mathbf{S}_{k}}^{s_{k}}\left(P_{\mathbb{C}}\right)\right) \widetilde{\psi}_{t}\left(\mathbf{R}_{i, j}\right)^{*}
$$

Taking the limits as $q_{1} \rightarrow \infty, \ldots, q_{k} \rightarrow \infty$, and using the identity

$$
I=\sum_{\left(s_{1}, \ldots, s_{k}\right) \in \mathbb{Z}_{+}^{k}} \Phi_{\mathbf{S}_{1}}^{s_{1}} \circ \cdots \circ \Phi_{\mathbf{S}_{k}}^{s_{k}}\left(\boldsymbol{\Delta}_{\mathbf{S}}(I)\right)=\sum_{\left(s_{1}, \ldots, s_{k}\right) \in \mathbb{Z}_{+}^{k}} \Phi_{\mathbf{S}_{1}}^{s_{1}} \cdots \circ \Phi_{\mathbf{S}_{k}}^{s_{k}}\left(P_{\mathbb{C}}\right),
$$

we deduce that

$$
\sum_{s_{1}=0}^{\infty} \cdots \sum_{s_{k}=0}^{\infty} \Phi_{\mathbf{S}_{1}}^{s_{1}} \circ \cdots \circ \Phi_{\mathbf{S}_{k}}^{s_{k}}\left(\Lambda_{t}\right)=\widetilde{\psi}_{t}\left(\mathbf{R}_{i, j}\right) \widetilde{\psi}_{t}\left(\mathbf{R}_{i, j}\right)^{*}
$$

Now, let $\left\{\Lambda_{t}\right\}_{t=1}^{N}$, where $N \in \mathbb{N}$ or $N=\infty$, be the set of the rank-one positive operators associated, as above, with all the operators $\Delta_{\mathcal{M}} Q_{\left(p_{1}, \ldots, p_{k}\right)}$ with $\left(p_{1}, \ldots, p_{k}\right) \in \Omega$, and let $\left\{\psi_{t}\right\}_{t=1}^{N}$ be the corresponding orthonormal multi-homogeneous polynomials, according to relation (3.2). We have $\Delta_{\mathcal{M}}=\sum_{t=1}^{N} \Lambda_{t}$ where the convergence in the strong operator topology, and $\operatorname{rank} \Delta_{\mathcal{M}}=N$. Note also that

$$
\Delta_{\mathcal{M}} \xi=\sum_{t=1}^{N}\left\langle\xi, \psi_{t}\right\rangle \psi_{t}, \quad \xi \in \otimes_{i=1}^{k} F^{2}\left(H_{n_{i}}\right)
$$

Due to the fact each completely positive map $\Phi_{\mathbf{S}_{i}}$ is $W O T$-continuous and using relation (3.3), we deduce that that

$$
\begin{aligned}
\sum_{s_{1}=0}^{q_{1}} \cdots \sum_{s_{k}=0}^{q_{k}} \Phi_{\mathbf{S}_{1}}^{s_{1}} \circ \cdots \circ \Phi_{\mathbf{S}_{k}}^{s_{k}}\left(\Delta_{\mathcal{M}}\right) & =\sum_{t=1}^{\infty}\left(\sum_{s_{1}=0}^{q_{1}} \cdots \sum_{s_{k}=0}^{q_{k}} \Phi_{\mathbf{S}_{1}}^{s_{1}} \circ \cdots \circ \Phi_{\mathbf{S}_{k}}^{s_{k}}\left(\Lambda_{t}\right)\right) \\
& =\sum_{t=1}^{\infty} \widetilde{\psi}_{t}\left(\mathbf{R}_{i, j}\right)\left(\sum_{s_{1}=0}^{q_{1}} \cdots \sum_{s_{k}=0}^{q_{k}} \Phi_{\mathbf{S}_{1}}^{s_{1}} \cdots \circ \Phi_{\mathbf{S}_{k}}^{s_{k}}\left(P_{\mathbb{C}}\right)\right) \widetilde{\psi}_{t}\left(\mathbf{R}_{i, j}\right)^{*} .
\end{aligned}
$$

Due to relation (3.1), $\left\{\sum_{s_{1}=0}^{q_{1}} \cdots \sum_{s_{k}=0}^{q_{k}} \Phi_{\mathbf{S}_{1}}^{s_{1}} \circ \cdots \circ \Phi_{\mathbf{S}_{k}}^{s_{k}}\left(\Delta_{\mathcal{M}}\right)\right\}$ is an increasing sequence of positive operators which converges strongly to $P_{\mathcal{M}}$ as $q_{1} \rightarrow \infty, \ldots, q_{k} \rightarrow \infty$, and $\left\{\sum_{s_{1}=0}^{q_{1}} \cdots \sum_{s_{k}=0}^{q_{k}} \Phi_{\mathbf{S}_{1}}^{s_{1}} \circ \cdots \circ \Phi_{\mathbf{S}_{k}}^{s_{k}}\left(P_{\mathbb{C}}\right)\right\}$ is an increasing sequence of positive operators which converges strongly to the identity operator on $\otimes_{i=1}^{k} F^{2}\left(H_{n_{i}}\right)$. Consequently, the equalities above and relation (3.3) can be used to deduce that

$$
P_{\mathcal{M}}=\sum_{t=1}^{\infty} \widetilde{\psi}_{t}\left(\mathbf{R}_{i, j}\right) \widetilde{\psi}_{t}\left(\mathbf{R}_{i, j}\right)^{*}
$$

where the convergence is in the strong operator topology. Let $\Psi:\left(\otimes_{i=1}^{k} F^{2}\left(H_{n_{i}}\right)\right) \otimes \mathbb{C}^{N} \rightarrow \otimes_{i=1}^{k} F^{2}\left(H_{n_{i}}\right)$ be the bounded operator having the $1 \times N$ matrix representation

$$
\left[\widetilde{\psi}_{1}\left(\mathbf{R}_{i, j}\right), \widetilde{\psi}_{2}\left(\mathbf{R}_{i, j}\right), \ldots\right]
$$

where $\mathbb{C}^{\infty}$ stands for $\ell^{2}(\mathbb{N})$. Note that $\Psi$ is a multi-analytic operator with respect to the universal model $\mathbf{S}$ and $\Psi \Psi^{*}=P_{\mathcal{M}}$. Therefore, $\Psi$ is a partial isometry. Since $\mathbf{S}_{i, j}$ are isometries, the initial space of $\Psi$, i.e.

$$
\Psi^{*}\left(\otimes_{i=1}^{k} F^{2}\left(H_{n_{i}}\right)\right)=\left\{x \in\left(\otimes_{i=1}^{k} F^{2}\left(H_{n_{i}}\right)\right) \otimes \mathbb{C}^{N}:\|\Psi x\|=\|x\|\right\}
$$

is reducing under each operator $\mathbf{S}_{i, j} \otimes I_{\mathbb{C}^{N}}$. Consequently, since $\boldsymbol{\Delta}_{\mathbf{S}}(I)=P_{\mathbb{C}}$, to prove that $\Psi$ is an isometry, it is enough to show that

$$
\mathbb{C}=P_{\mathbb{C}} \widetilde{\psi}_{t}\left(\mathbf{R}_{i, j}\right)^{*}\left(\otimes_{i=1}^{k} F^{2}\left(H_{n_{i}}\right)\right) \quad \text { for each } t \in\{1, \ldots, N\} .
$$


The latter equality is true due to the fact that

$$
P_{\mathbb{C}} \widetilde{\psi}_{t}\left(\mathbf{R}_{i, j}\right)^{*}\left(g_{t}\right)=\left\langle\widetilde{\psi}_{t}\left(\mathbf{R}_{i, j}\right)^{*}\left(\psi_{t}\right), 1\right\rangle=\left\|\psi_{t}\right\|^{2}=1
$$

The converse of the theorem is straightforward.

The next result is a Gauss-Bonnet-Chern type theorem for rank-one, graded, and pure elements in noncommutative polyballs.

Theorem 3.3. If $\mathbf{T}$ is a graded pure element of rank one in the noncommutative polyball $\mathbf{B}_{\mathbf{n}}(\mathcal{H})$, with $n_{i} \geq 2$, then

$$
\operatorname{curv}(\mathbf{T})=\chi(\mathbf{T})
$$

Proof. Let $U: \mathbb{T}^{k} \rightarrow B(\mathcal{H})$ be a strongly continuous unitary representation of the $k$-torus and assume that $\mathbf{T}$ is graded with respect to the gauge group $U$. The spectral subspaces of $U$ are

$$
\mathcal{H}_{\mathbf{s}}=\mathcal{H}_{\left(s_{1}, \ldots, s_{k}\right)}:=\left\{h \in \mathcal{H}: U\left(\lambda_{1}, \ldots, \lambda_{k}\right) h=\lambda_{1}^{s_{1}} \cdots \lambda_{k}^{s_{k}} h \text { for }\left(\lambda_{1} \ldots, \lambda_{k}\right) \in \mathbb{T}^{k}\right\},
$$

where $\mathbf{s}=\left(s_{1}, \ldots, s_{k}\right) \in \mathbb{Z}^{k}$. With respect to the orthogonal decomposition $\mathcal{H}=\bigoplus_{\mathbf{s} \in \mathbb{Z}^{k}} \mathcal{H}_{\mathbf{s}}$ we have

$$
U\left(\lambda_{1}, \ldots, \lambda_{k}\right)=\sum_{\left(s_{1}, \ldots, s_{k}\right) \in \mathbb{Z}^{k}} \lambda_{1}^{s_{1}} \cdots \lambda_{k}^{s_{k}} Q_{\left(s_{1}, \ldots, s_{k}\right)}, \quad\left(\lambda_{1} \ldots, \lambda_{k}\right) \in \mathbb{T}^{k},
$$

where $Q_{\left(s_{1}, \ldots, s_{k}\right)}$ is the orthogonal projection of $\mathcal{H}$ onto $\mathcal{H}_{\left(s_{1}, \ldots, s_{k}\right)}$. Note that if $\mathbf{T}$ is graded with respect to the gauge group $U$, then

$$
T_{i, j} \mathcal{H}_{\left(s_{1}, \ldots, s_{k}\right)} \subseteq \mathcal{H}_{\left(s_{1}, \ldots, s_{i-1}, s_{i}+1, s_{i+1}, \ldots, s_{k}\right)}, \quad\left(s_{1}, \ldots, s_{k}\right) \in \mathbb{Z}^{k},
$$

for any $i \in\{1, \ldots, k\}$ and $j \in\left\{1, \ldots, n_{i}\right\}$. Indeed, if $h \in \mathcal{H}_{\left(s_{1}, \ldots, s_{k}\right)}$, then $U\left(\lambda_{1}, \ldots, \lambda_{k}\right) h=\lambda_{1}^{s_{1}} \ldots \lambda_{k}^{s_{k}} h$ and

$$
U\left(\lambda_{1}, \ldots, \lambda_{k}\right) T_{i, j} h=\lambda_{i} T_{i, j} U\left(\lambda_{1}, \ldots, \lambda_{k}\right) h=\lambda_{1}^{s_{1}} \cdots \lambda_{i-1}^{s_{i-1}} \lambda_{i}^{s_{i}+1} \lambda_{i+1}^{s_{i+1}} \cdots \lambda_{k}^{s_{k}} T_{i, j} h
$$

for any $\left(\lambda_{1} \ldots, \lambda_{k}\right) \in \mathbb{T}^{k}$. Consequently, $T_{i, j} h \in \mathcal{H}_{\left(s_{1}, \ldots, s_{i-1}, s_{i}+1, s_{i+1}, \ldots, s_{k}\right)}$, which proves our assertion. Therefore,

$$
T_{i, j} Q_{\left(s_{1}, \ldots, s_{k}\right)}=Q_{\left(s_{1}, \ldots, s_{i-1}, s_{i}+1, s_{i+1}, \ldots, s_{k}\right)} T_{i, j}
$$

for any $\left(s_{1}, \ldots, s_{k}\right) \in \mathbb{Z}^{k}, i \in\{1, \ldots, k\}$, and $j \in\left\{1, \ldots, n_{i}\right\}$. This also implies

$$
Q_{\left(s_{1}, \ldots, s_{k}\right)} T_{i, j}^{*}=T_{i, j}^{*} Q_{\left(s_{1}, \ldots, s_{i-1}, s_{i}+1, s_{i+1}, \ldots, s_{k}\right)}
$$

Consequently, taking into account that $U\left(\lambda_{1}, \ldots, \lambda_{k}\right) Q_{\left(s_{1}, \ldots, s_{k}\right)}=\lambda_{1}^{s_{1}} \cdots \lambda_{k}^{s_{k}} Q_{\left(s_{1}, \ldots, s_{k}\right)}$ for any $\left(\lambda_{1}, \ldots, \lambda_{k}\right) \in$ $\mathbb{T}^{k}$, one can easily check that

$$
\boldsymbol{\Delta}_{\mathbf{T}}(I) U\left(\lambda_{1}, \ldots, \lambda_{k}\right) Q_{\left(s_{1}, \ldots, s_{k}\right)}=U\left(\lambda_{1}, \ldots, \lambda_{k}\right) Q_{\left(s_{1}, \ldots, s_{k}\right)} \boldsymbol{\Delta}_{\mathbf{T}}(I)
$$

for any $\left(s_{1}, \ldots, s_{k}\right) \in \mathbb{Z}^{k}$, which implies

$$
\boldsymbol{\Delta}_{\mathbf{T}}(I)^{1 / 2} U\left(\lambda_{1}, \ldots, \lambda_{k}\right)=U\left(\lambda_{1}, \ldots, \lambda_{k}\right) \boldsymbol{\Delta}_{\mathbf{T}}(I)^{1 / 2}, \quad\left(\lambda_{1}, \ldots, \lambda_{k}\right) \in \mathbb{T}^{k} .
$$

Due to the spectral theorem, we deduce that

$$
\boldsymbol{\Delta}_{\mathbf{T}}(I)^{1 / 2} Q_{\left(s_{1}, \ldots, s_{k}\right)}=Q_{\left(s_{1}, \ldots, s_{k}\right)} \boldsymbol{\Delta}_{\mathbf{T}}(I)^{1 / 2}, \quad\left(s_{1}, \ldots, s_{k}\right) \in \mathbb{Z}^{k} .
$$

Now, we assume that $\mathbf{T}$ has rank one. Then there exists $\left(d_{1}, \ldots, d_{k}\right) \in \mathbb{Z}_{+}^{k}$ such that

$$
\boldsymbol{\Delta}_{\mathbf{T}}(I)^{1 / 2}=Q_{\left(d_{1}, \ldots, d_{k}\right)} \boldsymbol{\Delta}_{\mathbf{T}}(I)^{1 / 2}=\boldsymbol{\Delta}_{\mathbf{T}}(I)^{1 / 2} Q_{\left(d_{1}, \ldots, d_{k}\right)} \neq 0
$$

and $\boldsymbol{\Delta}_{\mathbf{T}}(I)^{1 / 2} Q_{\left(s_{1}, \ldots, s_{k}\right)}=0$ for any $\left(s_{1}, \ldots, s_{k}\right) \in \mathbb{Z}^{k}$ with $\left(s_{1}, \ldots, s_{k}\right) \neq\left(d_{1}, \ldots, d_{k}\right)$. Consequently,

$$
\boldsymbol{\Delta}_{\mathbf{T}}(I)^{1 / 2}(\mathcal{H})=\boldsymbol{\Delta}_{\mathbf{T}}(I)^{1 / 2} \mathcal{H}_{\left(d_{1}, \ldots, d_{k}\right)} \subseteq \bigoplus_{s_{1} \geq d_{1}, \ldots, s_{k} \geq d_{k}} \mathcal{H}_{\left(s_{1}, \ldots, s_{k}\right)} .
$$


Since $\bigoplus_{s_{1} \geq d_{1}, \ldots, s_{k} \geq d_{k}} \mathcal{H}_{\left(s_{1}, \ldots, s_{k}\right)}$ is invariant under each operator $T_{i, j}$ for any $i \in\{1, \ldots, k\}$ and $j \in$ $\left\{1, \ldots, n_{i}\right\}$, we have

$$
T_{1, \beta_{1}} \cdots T_{k, \beta_{k}} \boldsymbol{\Delta}_{\mathbf{T}}(I)^{1 / 2} \mathcal{H} \subseteq \bigoplus_{s_{1} \geq d_{1}, \ldots, s_{k} \geq d_{k}} \mathcal{H}_{\left(s_{1}, \ldots, s_{k}\right)}
$$

for any $\beta_{i} \in \mathbb{F}_{n_{i}}^{+}, i=1, \ldots, k$. On the other hand, the noncommutative Berezin kernel

$$
\mathbf{K}_{\mathbf{T}}: \mathcal{H} \rightarrow F^{2}\left(H_{n_{1}}\right) \otimes \cdots \otimes F^{2}\left(H_{n_{k}}\right) \otimes \overline{\boldsymbol{\Delta}_{\mathbf{T}}(I)(\mathcal{H})}
$$

satisfies the relation

$$
\mathbf{K}_{\mathbf{T}}^{*}\left(e_{\beta_{1}}^{1} \otimes \cdots \otimes e_{\beta_{k}}^{k} \otimes h\right)=T_{1, \beta_{1}} \cdots T_{k, \beta_{k}} \boldsymbol{\Delta}_{\mathbf{T}}(I)^{1 / 2} h
$$

for any $\beta_{i} \in \mathbb{F}_{n_{i}}^{+}, i=1, \ldots, k$, and $h \in \mathcal{H}$. Since $\mathbf{T}$ is a pure element in the polyball, the Berezin kernel $\mathbf{K}_{\mathbf{T}}$ is an isometry and, therefore,

$$
\mathcal{H}=\overline{\operatorname{range} \mathbf{K}_{\mathbf{T}}^{*}}=\overline{\operatorname{span}}\left\{T_{1, \beta_{1}} \cdots T_{k, \beta_{k}} \boldsymbol{\Delta}_{\mathbf{T}}(I)^{1 / 2} h: \beta_{i} \in \mathbb{F}_{n_{i}}^{+}, h \in \mathcal{H}\right\} .
$$

Hence, and using relation (3.8), we deduce that

$$
\mathcal{H}=\bigoplus_{s_{1} \geq d_{1}, \ldots, s_{k} \geq d_{k}} \mathcal{H}_{\left(s_{1}, \ldots, s_{k}\right)} .
$$

On the other hand, taking into account relations (3.5), (3.6), and (3.7), we have

$$
\begin{aligned}
& \boldsymbol{\Delta}_{\mathbf{T}}(I)^{1 / 2} T_{k, \alpha_{k}}^{*} \cdots T_{1, \alpha_{k}}^{*} T_{1, \beta_{1}} \cdots T_{k, \beta_{k}} \boldsymbol{\Delta}_{\mathbf{T}}(I)^{1 / 2} Q_{\left(d_{1}, \ldots, d_{k}\right)} \\
& =\boldsymbol{\Delta}_{\mathbf{T}}(I)^{1 / 2} T_{k, \alpha_{k}}^{*} \cdots T_{1, \alpha_{k}}^{*} Q_{\left(d_{1}+\left|\beta_{1}\right|, \ldots, d_{k}+\left|\beta_{k}\right|\right)} T_{1, \beta_{1}} \cdots T_{k, \beta_{k}} \boldsymbol{\Delta}_{\mathbf{T}}(I)^{1 / 2} \\
& =\boldsymbol{\Delta}_{\mathbf{T}}(I)^{1 / 2} Q_{\left(d_{1}+\left|\beta_{1}\right|-\left|\alpha_{1}\right|, \ldots, d_{k}+\left|\beta_{k}\right|-\left|\alpha_{k}\right|\right)} T_{k, \alpha_{k}}^{*} \cdots T_{1, \alpha_{k}}^{*} T_{1, \beta_{1}} \cdots T_{k, \beta_{k}} \boldsymbol{\Delta}_{\mathbf{T}}(I)^{1 / 2} \\
& = \begin{cases}Q_{\left(d_{1}, \ldots, d_{k}\right)} \boldsymbol{\Delta}_{\mathbf{T}}(I)^{1 / 2} T_{k, \alpha_{k}}^{*} \cdots T_{1, \alpha_{k}}^{*} T_{1, \beta_{1}} \cdots T_{k, \beta_{k}} \boldsymbol{\Delta}_{\mathbf{T}}(I)^{1 / 2} & \text { if }\left|\beta_{1}\right|=\left|\alpha_{1}\right|, \ldots,\left|\beta_{k}\right|=\left|\alpha_{k}\right| \\
0 & \text { otherwise }\end{cases}
\end{aligned}
$$

for any $\alpha_{1}, \beta_{1} \in \mathbb{F}_{n_{1}}^{+}, \ldots, \alpha_{k}, \beta_{k} \in \mathbb{F}_{n_{k}}^{+}$. Consequently, we have

$$
\boldsymbol{\Delta}_{\mathbf{T}}(I)^{1 / 2} T_{k, \alpha_{k}}^{*} \cdots T_{1, \alpha_{k}}^{*} T_{1, \beta_{1}} \cdots T_{k, \beta_{k}} \boldsymbol{\Delta}_{\mathbf{T}}(I)^{1 / 2}=0
$$

for any $\alpha_{1}, \beta_{1} \in \mathbb{F}_{n_{1}}^{+}, \ldots, \alpha_{k}, \beta_{k} \in \mathbb{F}_{n_{k}}^{+}$, except when $\left|\beta_{1}\right|=\left|\alpha_{1}\right|, \ldots,\left|\beta_{k}\right|=\left|\alpha_{k}\right|$. Note that due to relations (3.9) and (3.10), for any $\left(q_{1}, \ldots, q_{k}\right) \in \mathbb{Z}_{+}^{k}$, we have

$$
\begin{aligned}
& \left(P_{q_{1}}^{(1)} \otimes \cdots \otimes P_{q_{k}}^{(k)} \otimes I_{\mathcal{H}}\right) \mathbf{K}_{\mathbf{T}} \mathbf{K}_{\mathbf{T}}^{*}\left(e_{\beta_{1}}^{1} \otimes \cdots \otimes e_{\beta_{k}}^{k} \otimes h\right) \\
& =\sum_{\alpha_{1} \in \mathbb{F}_{n_{1}}^{+},\left|\alpha_{1}\right|=q_{1}} \cdots \sum_{\alpha_{k} \in \mathbb{F}_{n_{k}}^{+},\left|\alpha_{k}\right|=q_{k}} e_{\alpha_{1}}^{1} \otimes \cdots \otimes e_{\alpha_{k}}^{k} \otimes \boldsymbol{\Delta}_{\mathbf{T}}(I)^{1 / 2} T_{k, \alpha_{k}}^{*} \cdots T_{1, \alpha_{k}}^{*} T_{1, \beta_{1}} \cdots T_{k, \beta_{k}} \boldsymbol{\Delta}_{\mathbf{T}}(I)^{1 / 2} h
\end{aligned}
$$

if $\left|\beta_{1}\right|=q_{1}, \ldots,\left|\beta_{k}\right|=q_{k}$, and zero otherwise. Now, one can see that

$$
\mathbf{K}_{\mathbf{T}} \mathbf{K}_{\mathbf{T}}^{*}\left(P_{q_{1}}^{(1)} \otimes \cdots \otimes P_{q_{k}}^{(k)} \otimes I_{\mathcal{H}}\right)=\left(P_{q_{1}}^{(1)} \otimes \cdots \otimes P_{q_{k}}^{(k)} \otimes I_{\mathcal{H}}\right) \mathbf{K}_{\mathbf{T}} \mathbf{K}_{\mathbf{T}}^{*}
$$

for any $\left(q_{1}, \ldots, q_{k}\right) \in \mathbb{Z}_{+}^{k}$, where $P_{q_{i}}^{(i)}$ is the orthogonal projection of the full Fock space $F^{2}\left(H_{n_{i}}\right)$ onto the span of all vectors $e_{\alpha_{i}}^{i}$ with $\alpha \in \mathbb{F}_{n_{i}}^{+}$and $\left|\alpha_{i}\right|=q_{i}$. Since $\mathbf{K}_{\mathbf{T}} \mathbf{K}_{\mathbf{T}}^{*}$ is a projection, we deduce that $\left.\mathbf{K}_{\mathbf{T}} \mathbf{K}_{\mathbf{T}}^{*}\left(P_{q_{1}}^{(1)} \otimes \cdots \otimes P_{q_{k}}^{(k)} \otimes I_{\mathcal{H}}\right)\right\}_{\left(q_{1}, \ldots, q_{k}\right) \in \mathbb{Z}_{+}^{k}}$ is a net of orthogonal projections with orthogonal ranges. This implies that

$$
\sum_{\substack{\left(s_{1}, \ldots, s_{k}\right) \in \mathbb{Z}_{+}^{k} \\\left(s_{1}, \ldots, s_{k}\right) \leq\left(q_{1}, \ldots, q_{k}\right)}} \mathbf{K}_{\mathbf{T}} \mathbf{K}_{\mathbf{T}}^{*}\left(P_{q_{1}}^{(1)} \otimes \cdots \otimes P_{q_{k}}^{(k)} \otimes I_{\mathcal{H}}\right)=\mathbf{K}_{\mathbf{T}} \mathbf{K}_{\mathbf{T}}^{*}\left(P_{\leq\left(q_{1}, \ldots, q_{k}\right)} \otimes I\right)
$$

is an orthogonal projection for any $\left(q_{1}, \ldots, q_{k}\right) \in \mathbb{Z}_{+}^{k}$. Consequently, we have

$$
\operatorname{trace}\left[\mathbf{K}_{\mathbf{T}} \mathbf{K}_{\mathbf{T}}^{*}\left(P_{\leq\left(q_{1}, \ldots, q_{k}\right)} \otimes I\right)\right]=\operatorname{rank}\left[\mathbf{K}_{\mathbf{T}} \mathbf{K}_{\mathbf{T}}^{*}\left(P_{\leq\left(q_{1}, \ldots, q_{k}\right)} \otimes I\right)\right] .
$$


Applying Theorem 1.2 and 24 (see Theorem 1.3), and using the fact that $\mathbf{K}_{\mathbf{T}}$ is an isometry, we deduce that

$$
\begin{aligned}
\operatorname{curv}(\mathbf{T}) & =\lim _{q_{1} \rightarrow \infty} \cdots \lim _{q_{k} \rightarrow \infty} \frac{\operatorname{trace}\left[\mathbf{K}_{\mathbf{T}}^{*}\left(P_{\leq\left(q_{1}, \ldots, q_{k}\right)} \otimes I\right) \mathbf{K}_{\mathbf{T}}\right]}{\operatorname{trace}\left[P_{\leq\left(q_{1}, \ldots, q_{k}\right)}\right]}=\lim _{q_{1} \rightarrow \infty} \cdots \lim _{q_{k} \rightarrow \infty} \frac{\operatorname{trace}\left[\mathbf{K}_{\mathbf{T}} \mathbf{K}_{\mathbf{T}}^{*}\left(P_{\leq\left(q_{1}, \ldots, q_{k}\right)} \otimes I\right)\right]}{\operatorname{trace}\left[P_{\leq\left(q_{1}, \ldots, q_{k}\right)}\right]} \\
& =\lim _{q_{1} \rightarrow \infty} \cdots \lim _{q_{k} \rightarrow \infty} \frac{\operatorname{rank}\left[\mathbf{K}_{\mathbf{T}} \mathbf{K}_{\mathbf{T}}^{*}\left(P_{\leq\left(q_{1}, \ldots, q_{k}\right)} \otimes I\right)\right]}{\operatorname{rank}\left[P_{\leq\left(q_{1}, \ldots, q_{k}\right)}\right]}=\lim _{q_{1} \rightarrow \infty} \cdots \lim _{q_{k} \rightarrow \infty} \frac{\operatorname{rank}\left[\mathbf{K}_{\mathbf{T}}^{*}\left(P_{\leq\left(q_{1}, \ldots, q_{k}\right)} \otimes I\right) \mathbf{K}_{\mathbf{T}}\right]}{\operatorname{rank}\left[P_{\leq\left(q_{1}, \ldots, q_{k}\right)}\right]} \\
& =\chi(\mathbf{T}) .
\end{aligned}
$$

The proof is complete.

Theorem 3.4. If $\mathcal{M}$ is a graded invariant subspace of the tensor product $F^{2}\left(H_{n_{1}}\right) \otimes \cdots \otimes F^{2}\left(H_{n_{k}}\right)$, then

$$
\lim _{q_{1} \rightarrow \infty} \ldots \lim _{q_{k} \rightarrow \infty} \frac{\operatorname{rank}\left[P_{\mathcal{M}^{\perp}} P_{\leq\left(q_{1}, \ldots, q_{k}\right)}\right]}{\operatorname{rank}\left[P_{\leq\left(q_{1}, \ldots, q_{k}\right)}\right]}=\lim _{q_{1} \rightarrow \infty} \ldots \lim _{q_{k} \rightarrow \infty} \frac{\operatorname{trace}\left[P_{\mathcal{M}^{\perp}} P_{\leq\left(q_{1}, \ldots, q_{k}\right)}\right]}{\operatorname{trace}\left[P_{\leq\left(q_{1}, \ldots, q_{k}\right)}\right]} .
$$

This is equivalent to

$$
\chi\left(\left.P_{\mathcal{M}^{\perp}} \mathbf{S}\right|_{\mathcal{M}^{\perp}}\right)=\operatorname{curv}\left(\left.P_{\mathcal{M}^{\perp}} \mathbf{S}\right|_{\mathcal{M}^{\perp}}\right) .
$$

Proof. Combining Theorem 2.4 with Theorem 3.3, and using Theorem 3.1 from [24, the result follows.

A closer look at the proof of Theorem 3.3. reveals that if $\mathbf{T} \in \mathbf{B}_{\mathbf{n}}(\mathcal{H})$ is a pure element of finite rank and

$$
\boldsymbol{\Delta}_{\mathbf{T}}(I)^{1 / 2} T_{k, \alpha_{k}}^{*} \cdots T_{1, \alpha_{k}}^{*} T_{1, \beta_{1}} \cdots T_{k, \beta_{k}} \boldsymbol{\Delta}_{\mathbf{T}}(I)^{1 / 2}=0
$$

for any $\alpha_{1}, \beta_{1} \in \mathbb{F}_{n_{1}}^{+}, \ldots, \alpha_{k}, \beta_{k} \in \mathbb{F}_{n_{k}}^{+}$, except when $\left|\beta_{1}\right|=\left|\alpha_{1}\right|, \ldots,\left|\beta_{k}\right|=\left|\alpha_{k}\right|$, then

$$
\operatorname{curv}(\mathbf{T})=\chi(\mathbf{T}) \text {. }
$$

Our final result is a version of the Gauss-Bonnet-Chern theorem for graded pure elements with finite rank in the noncommutative polyball.

Theorem 3.5. Let $\mathbf{T} \in \mathbf{B}_{\mathbf{n}}(\mathcal{H})$, with $n_{i} \geq 2$, be a pure element of finite rank which is graded with respect to a gauge group $U$, and let

$$
\mathcal{H}=\bigoplus_{\mathbf{s} \in \mathbb{Z}^{k}} \mathcal{H}_{\mathbf{s}}
$$

be the corresponding orthogonal decomposition. Then the spectral subspaces $\mathcal{H}_{\mathbf{s}}$ of $U$ are finite dimensional and there exist $\mathbf{c}, \mathbf{d} \in \mathbb{Z}^{k}$ with $\mathbf{c} \leq \mathbf{d}$ such that $\mathcal{H}=\bigoplus_{\mathbf{s} \in \mathbb{Z}^{k}, \mathbf{s} \geq \mathbf{c}} \mathcal{H}_{\mathbf{s}}$ and

$$
\mathcal{H}_{0}:=\bigoplus_{\mathbf{s} \in \mathbb{Z}^{k}, \mathbf{s} \geq \mathbf{d}} \mathcal{H}_{\mathbf{s}}
$$

is an invariant subspace of $\mathbf{T}$ with the property that $\left.\mathbf{T}\right|_{\mathcal{H}_{0}}$ is a finite rank element in $\mathbf{B}_{\mathbf{n}}\left(\mathcal{H}_{0}\right)$ and

$$
\operatorname{curv}\left(\left.\mathbf{T}\right|_{\mathcal{H}_{0}}\right)=\chi\left(\left.\mathbf{T}\right|_{\mathcal{H}_{0}}\right) .
$$

Proof. Assume that $\mathbf{T}$ is graded with respect to the gauge group

$$
U\left(\lambda_{1}, \ldots, \lambda_{k}\right)=\sum_{\left(s_{1}, \ldots, s_{k}\right) \in \mathbb{Z}^{k}} \lambda_{1}^{s_{1}} \cdots \lambda_{k}^{s_{k}} Q_{\left(s_{1}, \ldots, s_{k}\right)}, \quad\left(\lambda_{1} \ldots, \lambda_{k}\right) \in \mathbb{T}^{k} .
$$

The first part of the proof is similar to that of Theorem 3.3. Therefore, we can obtain that

$$
\boldsymbol{\Delta}_{\mathbf{T}}(I)^{1 / 2} Q_{\left(s_{1}, \ldots, s_{k}\right)}=Q_{\left(s_{1}, \ldots, s_{k}\right)} \boldsymbol{\Delta}_{\mathbf{T}}(I)^{1 / 2}, \quad\left(s_{1}, \ldots, s_{k}\right) \in \mathbb{Z}^{k} .
$$

Since $\boldsymbol{\Delta}_{\mathbf{T}}(I)^{1 / 2}$ is a positive finite rank operator in the commutatnt of $\left\{Q_{\left(s_{1}, \ldots, s_{k}\right)}\right\}_{\left(s_{1}, \ldots, s_{k}\right) \in \mathbb{Z}^{k}}$, we must have that $\boldsymbol{\Delta}_{\mathbf{T}}(I)^{1 / 2} Q_{\left(s_{1}, \ldots, s_{k}\right)}=0$ for all but finitely many $\left(s_{1}, \ldots, s_{k}\right) \in \mathbb{Z}^{k}$. Consequently, there are $\mathbf{c}=\left(c_{1}, \ldots, c_{k}\right) \leq \mathbf{d}=\left(d_{1}, \ldots, d_{k}\right)$ in $\mathbb{Z}^{k}$ such that

$$
\boldsymbol{\Delta}_{\mathbf{T}}(I)^{1 / 2}=\sum_{c_{1} \leq s_{1} \leq d_{1}, \ldots, c_{k} \leq s_{k} \leq d_{k}} D_{\left(s_{1}, \ldots, s_{k}\right)}^{1 / 2},
$$


where $D_{\left(s_{1}, \ldots, s_{k}\right)}^{1 / 2}:=\boldsymbol{\Delta}_{\mathbf{T}}(I)^{1 / 2} Q_{\left(s_{1}, \ldots, s_{k}\right)}$. Taking into account that $\bigoplus_{s_{1} \geq c_{1}, \ldots, s_{k} \geq c_{k}} \mathcal{H}_{\left(s_{1}, \ldots, s_{k}\right)}$ is invariant under each operator $T_{i, j}$ for any $i \in\{1, \ldots, k\}$ and $j \in\left\{1, \ldots, n_{i}\right\}$, we have

$$
T_{1, \beta_{1}} \cdots T_{k, \beta_{k}} \boldsymbol{\Delta}_{\mathbf{T}}(I)^{1 / 2} \mathcal{H} \subseteq \bigoplus_{s_{1} \geq c_{1}, \ldots, s_{k} \geq c_{k}} \mathcal{H}_{\left(s_{1}, \ldots, s_{k}\right)}
$$

for any $\beta_{i} \in \mathbb{F}_{n_{i}}^{+}$and $i \in\{1, \ldots, k\}$. Since $\mathbf{T}$ is a pure element in the polyball, the Berezin kernel $\mathbf{K}_{\mathbf{T}}$ is an isometry and

$$
\mathcal{H}=\overline{\text { range } \mathbf{K}_{\mathbf{T}}^{*}}=\overline{\operatorname{span}}\left\{T_{1, \beta_{1}} \cdots T_{k, \beta_{k}} \boldsymbol{\Delta}_{\mathbf{T}}(I)^{1 / 2} h: \beta_{i} \in \mathbb{F}_{n_{i}}^{+}, h \in \mathcal{H}\right\} .
$$

Hence, and using relation (3.12), we deduce that $\mathcal{H}=\bigoplus_{s_{1} \geq c_{1}, \ldots, s_{k} \geq c_{k}} \mathcal{H}_{\left(s_{1}, \ldots, s_{k}\right)}$. Due to relation (3.11) and the fact that $\boldsymbol{\Delta}_{\mathbf{T}}(I)^{1 / 2}$ has finite rank, we have

$$
\boldsymbol{\Delta}_{\mathbf{T}}(I)^{1 / 2} \mathcal{H} \subseteq \underset{c_{1} \leq s_{1} \leq d_{1}, \ldots, c_{k} \leq s_{k} \leq d_{k}}{\mathcal{M}_{\left(s_{1}, \ldots, s_{k}\right)} \subseteq} \bigoplus_{c_{1} \leq s_{1} \leq d_{1}, \ldots, c_{k} \leq s_{k} \leq d_{k}} \mathcal{H}_{\left(s_{1}, \ldots, s_{k}\right)},
$$

where $\mathcal{M}_{\left(s_{1}, \ldots, s_{k}\right)}:=\boldsymbol{\Delta}_{\mathbf{T}}(I)^{1 / 2} \mathcal{H}_{\left(s_{1}, \ldots, s_{k}\right)}$ is finite dimensional. On the other hand, if $\beta_{i} \in \mathbb{F}_{n_{i}}^{+}$with $\left|\beta_{i}\right|=m_{i} \in \mathbb{Z}_{+}$and $i \in\{1, \ldots, k\}$, we have

$$
T_{1, \beta_{1}} \cdots T_{k, \beta_{k}} \boldsymbol{\Delta}_{\mathbf{T}}(I)^{1 / 2} \mathcal{H} \subseteq \bigoplus_{c_{1}+m_{1} \leq s_{1} \leq d_{1}+m_{1}, \ldots, c_{k}+m_{k} \leq s_{k} \leq d_{k}+m_{k}} \mathcal{H}_{\left(s_{1}, \ldots, s_{k}\right)}
$$

and $T_{1, \beta_{1}} \cdots T_{k, \beta_{k}} \boldsymbol{\Delta}_{\mathbf{T}}(I)^{1 / 2} \mathcal{H}$ is a finite dimensional subspace. Consequently, using relation (3.13) and the fact that $\mathcal{H}=\bigoplus_{s_{1} \geq c_{1}, \ldots, s_{k} \geq c_{k}} \mathcal{H}_{\left(s_{1}, \ldots, s_{k}\right)}$, we conclude that all the spectral spaces $\mathcal{H}_{\left(s_{1}, \ldots, s_{k}\right)}$ are finite dimensional.

Since $\mathbf{T}$ is a pure element in $\mathbf{B}_{\mathbf{n}}(\mathcal{H})$, we have

$$
I=\sum_{p_{1}=0}^{\infty} \cdots \sum_{p_{k}=0}^{\infty} \Phi_{T_{1}}^{p_{1}} \circ \cdots \circ \Phi_{T_{k}}^{p_{k}}\left(\boldsymbol{\Delta}_{\mathbf{T}}(I)\right)
$$

where the convergence is in the weak operator topology. Hence, after multiplying to the left by $Q_{\left(q_{1}, \ldots, q_{k}\right)}$, we obtain

$$
Q_{\left(q_{1}, \ldots, q_{k}\right)}=\sum_{p_{1}=0}^{\infty} \cdots \sum_{p_{k}=0}^{\infty} Q_{\left(q_{1}, \ldots, q_{k}\right)} \Phi_{T_{1}}^{p_{1}} \circ \cdots \circ \Phi_{T_{k}}^{p_{k}}\left(\boldsymbol{\Delta}_{\mathbf{T}}(I)\right)
$$

for any $\left(q_{1}, \ldots, q_{k}\right) \in \mathbb{Z}^{k}$. On the other hand, taking into account relation (3.11), we deduce that

$$
Q_{\left(q_{1}, \ldots, q_{k}\right)} \Phi_{T_{1}}^{p_{1}} \circ \cdots \circ \Phi_{T_{k}}^{p_{k}}\left(\boldsymbol{\Delta}_{\mathbf{T}}(I)\right)=\sum_{s_{1}=c_{1}}^{d_{1}} \cdots \sum_{s_{k}=c_{k}}^{d_{k}} Q_{\left(q_{1}, \ldots, q_{k}\right)} \Phi_{T_{1}}^{p_{1}} \circ \cdots \circ \Phi_{T_{k}}^{p_{k}}\left(D_{\left(s_{1}, \ldots, s_{k}\right)}\right)
$$

for any $\left(p_{1}, \ldots, p_{k}\right) \in \mathbb{Z}_{+}^{k}$. Since $\boldsymbol{\Delta}_{\mathbf{T}}(I) \leq I$, it is clear that $D_{\left(s_{1}, \ldots, s_{k}\right)} \leq Q_{\left(s_{1}, \ldots, s_{k}\right)}$. Taking into account that

$$
T_{i, j} Q_{\left(s_{1}, \ldots, s_{k}\right)}=Q_{\left(s_{1}, \ldots, s_{i-1}, s_{i}+1, s_{i+1}, \ldots, s_{k}\right)} T_{i, j}
$$

for any $\left(s_{1}, \ldots, s_{k}\right) \in \mathbb{Z}^{k}, i \in\{1, \ldots, k\}$, and $j \in\left\{1, \ldots, n_{i}\right\}$, we have

$$
\begin{aligned}
\Phi_{T_{i}}\left(Q_{\left(s_{1}, \ldots, s_{k}\right)}\right) & =\sum_{j=1}^{n_{i}} T_{i, j} Q_{\left(s_{1}, \ldots, s_{k}\right)} T_{i, j}^{*} \\
& =Q_{\left(s_{1}, \ldots, s_{i-1}, s_{i}+1, s_{i+1}, \ldots, s_{k}\right)} \Phi_{T_{i}}(I) Q_{\left(s_{1}, \ldots, s_{i-1}, s_{i}+1, s_{i+1}, \ldots, s_{k}\right)} \\
& \leq Q_{\left(s_{1}, \ldots, s_{i-1}, s_{i}+1, s_{i+1}, \ldots, s_{k}\right)}
\end{aligned}
$$

for any $i \in\{1, \ldots, k\}$. Consequently, and using the inequality $D_{\left(s_{1}, \ldots, s_{k}\right)} \leq Q_{\left(s_{1}, \ldots, s_{k}\right)}$, we deduce that

$$
\Phi_{T_{1}}^{p_{1}} \circ \cdots \circ \Phi_{T_{k}}^{p_{k}}\left(D_{\left(s_{1}, \ldots, s_{k}\right)}\right) \leq \Phi_{T_{1}}^{p_{1}} \circ \cdots \circ \Phi_{T_{k}}^{p_{k}}\left(Q_{\left(s_{1}, \ldots, s_{k}\right)}\right) \leq Q_{\left(s_{1}+p_{1}, \ldots, s_{k}+p_{k}\right)}
$$


for any $\left(p_{1}, \ldots, p_{k}\right) \in \mathbb{Z}_{+}^{k}$. Now, let $\left(q_{1}, \ldots, q_{k}\right) \in \mathbb{Z}^{k}$ be such that $\left(q_{1}, \ldots, q_{k}\right) \geq\left(d_{1}, \ldots, d_{k}\right)$, Since $\left\{Q_{\left(s_{1}, \ldots, s_{k}\right)}\right\}$ are orthogonal projections, relation (3.16) implies

$$
\begin{aligned}
\sum_{p_{1}=0}^{\infty} \cdots & \sum_{p_{k}=0}^{\infty} \sum_{s_{1}=c_{1}}^{d_{1}} \cdots \sum_{s_{k}=c_{k}}^{d_{k}} Q_{\left(q_{1}, \ldots, q_{k}\right)} \Phi_{T_{1}}^{p_{1}} \circ \cdots \circ \Phi_{T_{k}}^{p_{k}}\left(D_{\left(s_{1}, \ldots, s_{k}\right)}\right) \\
& =\sum_{s_{1}=c_{1}}^{d_{1}} \cdots \sum_{s_{k}=c_{k}}^{d_{k}} \Phi_{T_{1}}^{q_{1}-s_{1}} \circ \cdots \circ \Phi_{T_{k}}^{q_{k}-s_{k}}\left(D_{\left(s_{1}, \ldots, s_{k}\right)}\right) \\
& =\Phi_{T_{1}}^{q_{1}-d_{1}} \circ \cdots \circ \Phi_{T_{k}}^{q_{k}-d_{k}}\left(\sum_{s_{1}=c_{1}}^{d_{1}} \cdots \sum_{s_{k}=c_{k}}^{d_{k}} \Phi_{T_{1}}^{d_{1}-s_{1}} \circ \cdots \circ \Phi_{T_{k}}^{d_{k}-s_{k}}\left(D_{\left(s_{1}, \ldots, s_{k}\right)}\right)\right) .
\end{aligned}
$$

Due to relations (3.14) and (3.15), we obtain

$$
Q_{\left(q_{1}, \ldots, q_{k}\right)}=\Phi_{T_{1}}^{q_{1}-d_{1}} \circ \cdots \circ \Phi_{T_{k}}^{q_{k}-d_{k}}(B)
$$

where

$$
B:=\sum_{s_{1}=c_{1}}^{d_{1}} \cdots \sum_{s_{k}=c_{k}}^{d_{k}} \Phi_{T_{1}}^{d_{1}-s_{1}} \circ \cdots \circ \Phi_{T_{k}}^{d_{k}-s_{k}}\left(D_{\left(s_{1}, \ldots, s_{k}\right)}\right) .
$$

Due to relation (3.17), we deduce that

$$
\Phi_{T_{i}}\left(Q_{\left(q_{1}, \ldots, q_{k}\right)}\right)=Q_{\left(q_{1}, \ldots, q_{i-1}, q_{i}+1, q_{i+1}, \ldots, q_{k}\right)}
$$

for any $\left(q_{1}, \ldots, q_{k}\right) \geq\left(d_{1}, \ldots, d_{k}\right)$ and $i \in\{1, \ldots, k\}$. Consider the subspace

$$
\mathcal{H}_{0}:=\bigoplus_{s_{1} \geq d_{1}, \ldots, s_{k} \geq d_{k}} Q_{\left(s_{1}, \ldots, s_{k}\right)} \mathcal{H}
$$

and let $\left.\mathbf{T}\right|_{\mathcal{H}_{0}}:=\left(\left.T_{1}\right|_{\mathcal{H}_{0}},\left.\ldots T_{k}\right|_{\mathcal{H}_{0}}\right)$ with $\left.T_{i}\right|_{\mathcal{H}_{0}}:=\left(\left.T_{i, 1}\right|_{\mathcal{H}_{0}}, \ldots,\left.T_{i, n_{i}}\right|_{\mathcal{H}_{0}}\right)$ for $i \in\{1, \ldots, k\}$. An inductive argument shows that, for any $\left(m_{1}, \ldots, m_{k}\right) \in \mathbb{Z}_{+}^{k}$,

$$
\begin{aligned}
& \left(i d-\Phi_{T_{1} \mid \mathcal{H}_{0}}^{m_{1}+1}\right) \circ \cdots \circ\left(i d-\Phi_{T_{k} \mid \mathcal{H}_{0}}^{m_{k}+1}\right)\left(I_{\mathcal{H}_{0}}\right) \\
& =\left(i d-\Phi_{T_{2} \mid \mathcal{H}_{0}}^{m_{2}+1}\right) \circ \cdots \circ\left(i d-\Phi_{T_{k} \mid \mathcal{H}_{0}}^{m_{k}+1}\right)\left(\sum_{d_{1} \leq s_{1} \leq d_{1}+m_{1}} \sum_{s_{2}=d_{2}}^{\infty} \cdots \sum_{s_{k}=d_{k}} Q_{\left(s_{1}, s_{2}, \ldots, s_{k}\right) \mid \mathcal{H}_{0}}\right) \\
& =\cdots=\left(i d-\Phi_{T_{k} \mid \mathcal{H}_{0}}^{m_{k}+1}\right)\left(\left.\sum_{d_{1} \leq s_{1} \leq d_{1}+m_{1}} \cdots \sum_{d_{k-1} \leq s_{k-1} \leq d_{k-1}+m_{k-1}} \sum_{s_{k}=d_{k}} Q_{\left(s_{1}, s_{2}, \ldots, s_{k-1}, s_{k}\right)}\right|_{\mathcal{H}_{0}}\right) \\
& =\left.\sum_{d_{1} \leq s_{1} \leq d_{1}+m_{1}} \ldots \sum_{d_{k} \leq s_{k} \leq d_{k}+m_{k}} Q_{\left(s_{1}, \ldots, s_{k}\right)}\right|_{\mathcal{H}_{0} .} .
\end{aligned}
$$

In particular, for $m_{1}=\cdots=m_{k}=1$, we have

$$
\left(i d-\Phi_{T_{1} \mid \mathcal{H}_{0}}\right) \circ \cdots \circ\left(i d-\Phi_{T_{k} \mid \mathcal{H}_{0}}\right)\left(I_{\mathcal{H}_{0}}\right)=\left.Q_{\left(d_{1}, d_{2}, \ldots, d_{k}\right)}\right|_{\mathcal{H}_{0}} \geq 0
$$

which shows that $\left.\mathbf{T}\right|_{\mathcal{H}_{0}} \in \mathbf{B}_{\mathbf{n}}\left(\mathcal{H}_{0}\right)$ and has finite rank. On the other hand, due to the relations above, the operator $\left(i d-\Phi_{\left.T_{1}\right|_{\mathcal{H}_{0}}}^{m_{1}+1}\right) \circ \cdots \circ\left(i d-\Phi_{\left.T_{k}\right|_{\mathcal{H}_{0}}}^{m_{k}+1}\right)\left(I_{\mathcal{H}_{0}}\right)$ is an orthogonal projection for any $\left(m_{1}, \ldots, m_{k}\right) \in \mathbb{Z}_{+}^{k}$. Therefore,

$$
\frac{\operatorname{trace}\left[\left(i d-\Phi_{T_{1}}^{m_{1}+1}\right) \circ \cdots \circ\left(i d-\Phi_{T_{k}}^{m_{k}+1}\right)(I)\right]}{\prod_{i=1}^{k}\left(1+n_{i}+\cdots+n_{i}^{m_{i}}\right)}=\frac{\operatorname{rank}\left[\left(i d-\Phi_{T_{1}}^{m_{1}+1}\right) \circ \cdots \circ\left(i d-\Phi_{T_{k}}^{m_{k}+1}\right)(I)\right]}{\prod_{i=1}^{k}\left(1+n_{i}+\cdots+n_{i}^{m_{i}}\right)} .
$$

Consequently, applying Theorem 1.2 and using [24] (see Theorem 1.3 and Corollary 1.4), we deduce that $\operatorname{curv}\left(\left.\mathbf{T}\right|_{\mathcal{H}_{0}}\right)=\chi\left(\left.\mathbf{T}\right|_{\mathcal{H}_{0}}\right)$. This completes the proof. 


\section{REFERENCES}

[1] W.B. Arveson, Subalgebras of $C^{*}$-algebras III: Multivariable operator theory, Acta Math. 181 (1998), $159-228$.

[2] W.B. Arveson, The curvature invariant of a Hilbert module over $\mathbb{C}\left[z_{1}, \ldots, z_{n}\right]$, J. Reine Angew. Math. 522 (2000), $173-236$.

[3] W.B. Arveson, The Dirac operator of a commuting $d$-tuple, J. Funct. Anal. 189 (2002), 53-79.

[4] A. Beurling, On two problems concerning linear transformations in Hilbert space, Acta Math. 81 (1948), $239-251$.

[5] R.G. Douglas and V.I. Paulsen, Hilbert modules over function algebras, Pitman Research Notes in Mathematics Series, vol.217, 1989.

[6] X. FAnG, Hilbert polynomials and Arveson's curvature invariant, J. Funct. Anal. 198 (2003), no. 2, $445-464$.

[7] X. FAng, Invariant subspaces of the Dirichlet space and commutative algebra, J. Reine Angew. Math. 569 (2004), $189-211$.

[8] X. FAng, Additive invariants on the Hardy space over the polydisc, J. Funct. Anal. 253 (2007), no. 1, 359-372.

[9] D. Greene, S. Richter, And C Sundberg, The structure of inner multipliers on spaces with complete Nevanlinna-Pick kernels, J. Funct. Anal. 194 (2002), 311-331.

[10] J. Gleason, S. Richter, and C Sundberg, On the index of invariant subspaces in spaces of analytic functions of several complex variables, J. Reine Angew. Math. 587 (2005), 49-76.

[11] I. Kaplansky, Commutative rings, Allyn and Bacon, Boston, 1970.

[12] D.W. KRIBS, The curvature invariant of a non-commuting n-tuple, Integral Equations Operator Theory 41 (2001), $426-454$.

[13] P.S. Muhly And B. Solel, The curvature and index of completely positive maps, Proc. London Math. Soc. (3) 87 (2003).

[14] V.I. Paulsen, Completely Bounded Maps and Dilations, Pitman Research Notes in Mathematics, Vol.146, New York, 1986.

[15] G. Popescu, Characteristic functions for infinite sequences of noncommuting operators, J. Operator Theory 22 (1989), $51-71$.

[16] G. Popescu, Multi-analytic operators on Fock spaces, Math. Ann. 303 (1995), 31-46.

[17] G. Popescu, Poisson transforms on some $C^{*}$-algebras generated by isometries, J. Funct. Anal. 161 (1999), 27-61.

[18] G. Popescu, Curvature invariant for Hilbert modules over free semigroup algebras, Adv. Math. 158 (2001), 264-309.

[19] G. Popescu, Operator theory on noncommutative varieties, Indiana Univ. Math. J. 55 (2) (2006), 389-442.

[20] G. Popescu, Operator theory on noncommutative domains, Mem. Amer. Math. Soc. 205 (2010), no. 964, vi+124 pp.

[21] G. Popescu, Free holomorphic automorphisms of the unit ball of $B(\mathcal{H})^{n}$, J. Reine Angew. Math., 638 (2010), 119-168.

[22] G. Popescu, Berezin transforms on noncommutative polydomains, Trans. Amer. Math. Soc., to appear.

[23] G. Popescu, Berezin transforms on noncommutative varieties in polydomains, J. Funct. Anal. 265 (2013), no. 10, 2500-2552.

[24] G. Popescu, Curvature invariant on noncommutative polyballs, submitted for publication.

[25] S. Richter, Invariant subspaces of the Dirichlet shift, J. Reine Angew. Math. 386 (1988), 205-220.

[26] W. Rudin, Function theory in polydiscs, W. A. Benjamin, Inc., New York-Amsterdam 1969 vii+188 pp.

Department of Mathematics, The University of Texas at San Antonio, San Antonio, TX 78249, USA

E-mail address: gelu.popescu@utsa.edu 